\title{
A.K.Xusanov
}

\section{HAYVONLAR EKOLOGIYASI}

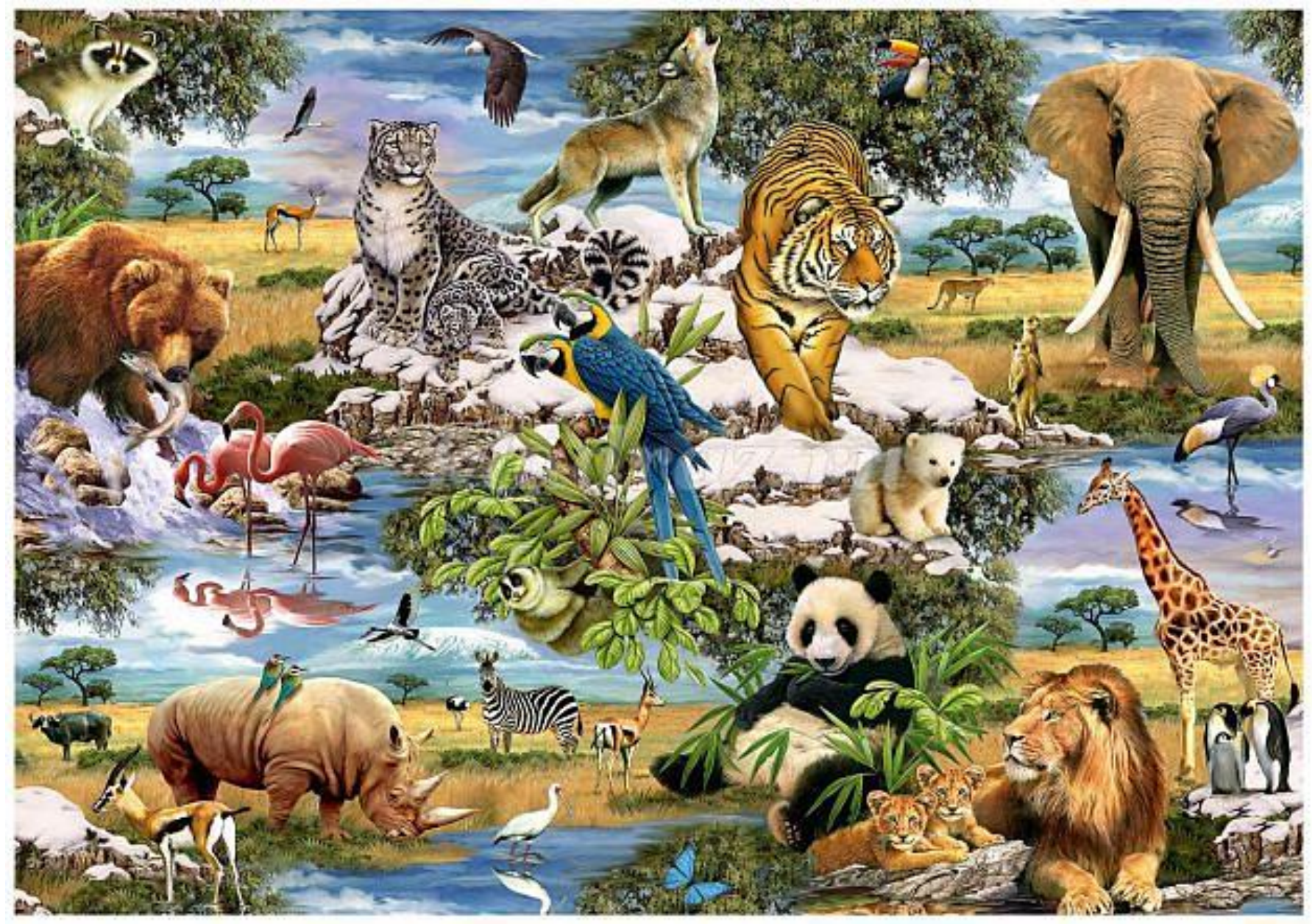

Андижон - 2021 
O'ZBEKISTON RESPUBLIKASI

OLIY VA O'RTA MAXSUS TA'LIM VAZIRLIGI

ANDIJON DAVLAT UNIVERSITETI

A.K.XUSANOV

\section{HAYVONLAR EKOLOGIYASI}

Oliy o'quv yurtlarining «biologiya», «ekologiya va atrof muhit muxofazasi» yo'nalishi magistr va talabalari uchun

Andijon - 2021 
Mazkur o'quv qo'llanma oliy o'quv yurtlarining «biologiya», «ekologiya va atrof muhit muxofazasi» yo'nalishlari magistr va talabalari uchun mo'ljallangan bo'lib, Andijon davlat universiteti, zoologiya va biokimyo kafedrasi yig'ilishida, Tabiiy fanlar fakulteti va universitet ilmiy kengashlarida muhokama qilingan hamda chop etishga tavsiya etilgan.

\title{
Taqrizchilar:
}

Farg'ona davlat universiteti tabiiy fanlar fakulteti dekani, b.f.d., dosent I.I.Zokirov

Andijon davlat universiteti zoologiya va biokimyo kafedrasi dotsenti, b.f.n. A.Ummatov

\author{
Hayvonlar ekologiyasi (o'quv qo'lanma) \\ A.Xusanov - Andijon. ADU, 2021. - 125 b.
}

ISBN 978-985-528-511-4.

Qo'llanmada hayvonlar ekologiyasi fani va uning bo'limlari, ekologiyada foydalaniladigan uslublar, fanning qisqacha tarixi, vazifalari, Markaziy Osiyo olimlarining tabiat va ekologiyaga oid fikrlari, O'zbekistonda hayvonlar ekologiyasi fanining qisqacha rivojlanish tarixi, hayvonlarning yashash muhitlari va adaptatsiyalar, muhit omillarining ta'sir etishini umumiy qonuniyatlari, biologik maromlar, hayvonlarning hayotiy shakllari, populyatsiyalar ekologiyasi, biotsenoz, biogeotsenoz va ekotizimlar hamda biosferada hayvonlarning tarqalish chegaralari to'g'risidagi ma'lumotlar yoritilgan.

UDK 591.5

Andijon davlat universiteti, 2021

O'zbekiston Respublikasi Oliy va o'rta maxsus ta'lim vazirligining 2021 yil 25 dekabrdagi 538 - sonli buyrug'iga asosan nashrga ruxsat etilgan. 


\section{KIRISH}

Ushbu o'quv qo'llanma
professor Madaminbek Hotamovich Ahmedovning yorqin xotirasiga bag'ishlanadi

Hayvonot dunyosi biosferaning tarkibiga kirib, ekologik tizim zanjirining ajralmas qismi hisoblanadi. Ular tabiatda modda almashinuvi jarayonining zaruriy komponenti bo'lib, tabiiy jamoalarning faoliyat ko'rsatishini, tabiiy muhitning boshqa elementlari - tuproq unumdorligi, o'simlik qoplamining shakllanishini ta'minlaydi.

Oxirgi ma'lumotlarga ko'ra O'zbekiston faunasi tarkibiga 15 mingdan ziyod umurtqasiz hayvonlar, 714 turdagi umurtqali hayvonlar, jumladan, 107 tur sut emizuvchilar, 467 tur qushlar, 61 tur sudralib yuruvchilar, 76 tur baliqlar va 3 tur amfibiyalar kiradi.

Hayvonot dunyosini muhofaza qilishda qo'riqxonalar, zakazniklar (buyurtmaxonalar) va “Qizil Kitob”ning ahamiyati katta bo'lib, O'zbekiston “Qizil Kitobi”ning I-nashriga hayvonlarning 187 turi kiritilgan bo'lsa, uning IInashriga 206 tur kiritildi.

Keyingi yillarda Respublikamizda muhofaza qilinishi lozim bo'lgan hayvonlar va o'simliklar turlariga alohida e'tibor qaratilib muhofaza choratadbirlari ishlab chiqilmoqda.

Mustaqillik yillarida mamlakatimizda barcha jabhalar qatorida hayvonot dunyosini muhofaza etish, ekologiya, atrof-muhitni muhofaza qilish va tabiiy resurslardan oqilona foydalanish sohasida to'laqonli huquqiyme'yoriy baza yaratildi. Xususan, 1997 yili qabul qilingan O'zbekiston Respublikasining «Hayvonot dunyosini muhofaza qilish va undan foydalanish to'g'risida»gi qonuni mamlakatimiz hududidagi hayvonot olami, shu jumladan, ko'chib yuruvchi yovvoyi hayvonlar populyatsiyalarini muhofaza qilish, ulardan oqilona foydalanish va qayta tiklash, shuningdek, yovvoyi hayvonlarning yashash muhitini saqlab qolish sohasidagi munosabatlarni tartibga soladi.

2016 yil 24 avgustdagi “O'simlik va hayvonot dunyosini muhofaza qilish va undan oqilona foydalanish" to'g'risidagi, yangi tahrirdagi qonun Senat tomonidan tasdiqlandi. Ushbu qonunning maqsadi hayvonot va o'simliklar 
dunyosini muhofaza qilish va undan foydalanish sohasidagi munosabatlarni tartibga solishdan iborat.

Shu bilan birga, o'tgan davr mobaynida O'zbekiston biologik xilma xillikni saqlash nuqtai nazaridan xalqaro munosabatlarni integratsiyalashish maqsadida, bir qator muhim xalqaro ekologik normativ-huquqiy hujjatlarga qo'shildi. Ular qatoriga «yo'qolib ketish xavfi ostidagi yovvoyi fauna va flora turlari bilan xalqaro savdo qilish to'g'risida»gi (CITES); «Yovvoyi hayvonlarning ko'chib yuruvchi turlarini muhofaza qilish to'g'risida»gi; «Asosan suvda suzuvchi qushlar yashash joylari sifatida xalqaro ahamiyatga ega bo'lgan suv-botqoq joylari to'g'risida»gi konventsiyalarni, hamda AfrikaYevroosiyoning ko'chib yuruvchi suv-botqoq qushlarining muhofazasi bo'yicha bitimlarni kiritish mumkin.

Ta'kidlash joizki, O'zbekiston Respublikasining Konstitutsiyasi, “Tabiatni muhofaza qilish to'g'risida"gi, “Ta'lim to'g'risida"gi qonunlari, shuningdek, boshqa qator hujjatlar ekologik ta'lim-tarbiya tizimining huquqiy asosini tashkil etadi. Jumladan, “Tabiatni muhofaza qilish to'g'risida”gi O’zbekiston Respublikasi Qonunining 4-moddasida tabiatni muhofaza qilish maqsadlariga erishish uchun barcha turdagi ta'lim muassasalarida ekologiya fanini o'qitishning majburiyligini ta'minlash mustahkamlab qo'yilgan. Bu esa, "Hayvonlar ekologiyasi" kursining oliygoh talabalariga o'qitilishi ham bir tomondan Davlat ta'lim standartlarining bajarilishi bo'lsa, ikkinchi tomondan, tabiatdan oqilona foydalanishni va tabiatni muhofaza qilish sohasiga doir huquqiy normalarning amaliy ijrosi sanaladi.

Ushbu havola etilayotgan mazkur qo'llanmani yaratish g'oyasi dastlab taniqli zoolog olim, professor M.H.Ahmedov tomonidan ilgari surilgan. O'quv adabiyoti u kishining shogirdlari tomonidan nihoyasiga etkazilib, o'quvchilarga taqdim etilmoqda.

Unda hayvonlar ekologiyasining fan sifatida shakllanishidan tortib to autoekologiya, sinekologiya hayvonlarning asosiy yashash muhitlari, biotoplar bo'yicha taqsimlanishi, ularga moslanishlari, populyatsiyalar ekologiyasi, biotsenoz, biogeotsenoz va ekotizimlar hamda biosferada hayvonlarning tarqalish masalalari xususida so'z yuritilgan.

Risola biologiya va ekologiya sohasida ta'lim olayotgan talabalarga, magistrantlar, ilmiy va amaliyot xodimlariga mo'ljallangan. 
Muallif hamkasblarni risola haqida bildirgan qimmatli fikr-mulohazalarini samimiyat bilan qabul qiladi, zero ularning barchasi keyingi tadqiqotlarga ijobiy yo'nalish berishi shubhasiz.

Muallif 


\section{1-BO'LIM. HAYVONLAR EKOLOGIYASI FAN SIFATIDA Ekologiya fani va uning bo'limlari}

Organizm va muhit o'rtasidagi o'zaro munosabatlarni barcha biologiya fanlari ma'lum yo'nalishda talqin etsa-da, ekologiya fani esa mavjudotlarni yashash muhiti bilan bo'lgan o'zaro munosabatlari majmuida batafsil va atroflicha o'rganadi hamda tahlil etadi. Shuning uchun ham grekcha "esos" yoki "oikos" - uy, uya, yashash joyi, "logos"- bilim, ta'limot, fan degan ma'noni beradi.

Ekologiya tirik organizmlarning yashash sharoitlari, ularning o'zi yashab turgan muhit bilan o'zaro murakkab, keng qamrovli munosabatlarini va shu asosda vujudga kelgan qonuniyatlarni o'rganadi. «Ekologiya» tushunchasi nemis biologi E.Gekkel tomonidan fanga 1866 yilda kiritilgan va uning «Genegelle Mogrhologie der Organizmen» asarida ilk marta qo'llanilgan.

Ekologiya organizmlarning yashash muhiti bilan bo'lgan bevosita munosabatlarinigina o'rganib qolmay, balki mazkur munosabatlarning tarixiy shakllanishi asosida:

- organizmlarning yashash muhitiga xususiy hamda tarixiy moslanishi;

- tur ichidagi munosabatlar, organizmlarning ko'payishi, tarqalishi va yashash tarzi, shuningdek miqdor zichliklarining o'zgarishi;

- populyatsiyalarning o'ziga xos xususiyatlari va strukturasining turli ekosistemalar ozuqa zanjiri hamda funktsiyalanishida alohida turlarning o'rnini va ahamiyatini ham o'rganadi.

Organizmlar populyatsiyalar bilan bog'liq, turlar miqdor zichligining o'zgarishini tahlil etish, uning tarkibini o'rganish imkonini beradi. Populyatsiyalar, turlar, biotsenozlar, biogeotsenozlar, ekotizimlar va biosfera tushunchalari ekologiya fanining tayanch manbaini tashkil etadi.

Umumiy ekologiya 4 bo'limdan iborat: autoekologiya, populyatsiyalar ekologiyasi, sinekologiya va biosfera.

Autoekologiya (Schroter, 1896) yakka - tur individlarining atrof muhit bilan o'zaro munosabatlarini o'rganadi. Organizmlarning ekologik omillarga bo'lgan talabi, chidamlilik darajasi, ulardagi morfologik, fiziologik 
moslanishlarni va fe'l-atvor reaktsiyalarini tahlil etadi.

Populyatsiyalar ekologiyasi (Schwertfeger, 1963) populyatsiyalar tuzilmasi, dinamikasi yoki sur'atlarini, turlarning miqdor zichliklarini vaqt birligida (sutkalik, mavsumiy, yillik) o'zgarishini va bu holatning sabablarini talqin etadi.

Sinekologiya (Games, 1918) biogeotsenozlardagi ko'pchilik turlarning o'zaro, shuningdek organizmlarning atrof-muhit bilan bo'lgan munosabatlarini o'rganadi. "Biotsenologiya" atamasi amalda sinekologiyaning sinonimi hisoblanadi.

Biosfera (Zyuss, 1875) - tirik organizmlarning tarqalish "sferasi"sayyoramizda tarqalgan organizmlar, ya'ni er qobig'idagi mavjudotlar tizimi. Ekotizimlarni tadqiq qilishning kengayishi va rivojlanishi biosfera haqidagi ta'limotni shakllanishiga olib keldi. Vernadskiy (1926) biosfera ta'limotini asosladi.

Ekologiyaning mazkur omillari organizmlarni turli darajada (yakka, populyatsiya va jamoa) yashash muhiti bilan bo'lgan munosabatlari qonuniyatlarini talqin qilish imkoniyatini beradi. Uning boshqa bo'limlari biosfera doirasida dengiz, quruqlik va chuchuk suv ekotizimlarini tadqiq etadi.

Ekologiyani o'simlik va hayvonlar ekologiyasiga ajratish bir muncha shartli bo'lib, qator umumbiologik yo'nalishlar ikkalasi uchun ham xosdir.

Shu bilan birga, hayvonlar ekologiyasi fani zoologiya tarkibidagi mustaqil fan sanaladi. O'z navbatida, hayvonlar ekologiyasi bir qancha bo'limlarga ajraladi: a) suv hayvonlari ekologiyasi - gidrobiologiya; b) tuproqda yashovchi hayvonlar ekologiyasi yoki pedobiologiya; v) quruqlik hayvonlari ekologiyasi; g) parazit turlar ekologiyasi - parazitologiya (1jadval). 


\section{Ekologiya bo'limlari}

1 -jadval

\begin{tabular}{|c|c|c|c|c|c|}
\hline \multicolumn{2}{|c|}{ EKOLOGIYA } & $\begin{array}{l}\text { Suv hayvonlari } \\
\text { (gidrobionlar) } \\
\text { ekologiyasi }\end{array}$ & $\begin{array}{l}\text { Tuproqda } \\
\text { yashovchi } \\
\text { hayvonlar } \\
\text { (pedobiontlar) } \\
\text { ekologiyasi } \\
\end{array}$ & $\begin{array}{l}\text { Quruqlik } \\
\text { hayvonlari } \\
\text { (aerobiontlar) } \\
\text { ekologiyasi }\end{array}$ & $\begin{array}{l}\text { Parazitlar } \\
\text { ekologiyasi }\end{array}$ \\
\hline $\begin{array}{l}\text { Individlar } \\
\text { ekologiyasi }\end{array}$ & \multirow{2}{*}{ 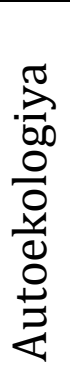 } & \multicolumn{4}{|c|}{$\begin{array}{l}\text { Individlarning tashqi omillarga javob reaktsiyalarini yashash } \\
\text { muhitiga moslanishi xususiyatlarini o'rganish }\end{array}$} \\
\hline $\begin{array}{l}\text { Populyatsiyalar } \\
\text { ekologiyasi }\end{array}$ & & \multicolumn{4}{|c|}{$\begin{array}{l}\text { Populyatsiyalarning muhit bilan bo'lgan o'ziga xos } \\
\text { munosabatlarini, ularning shakllanishi, tuzilmasi va sur'atlarini } \\
\text { o'rganish }\end{array}$} \\
\hline $\begin{array}{l}\text { Jamoalar } \\
\text { ekologiyasi } \\
\text { (biotsenologiya) }\end{array}$ & $\stackrel{5}{\overparen{20}}$ & \multicolumn{4}{|c|}{$\begin{array}{l}\text { Biotsenozlarning shakllanishi, tuzilmasi va sur'atlarini o'rganish, } \\
\text { ularni muhit bilan bo'lgan munosabatlarini izohlash }\end{array}$} \\
\hline Biosfera & 坛 & \multicolumn{4}{|c|}{$\begin{array}{l}\text { Yerning tirik organizmlar yashaydigan alohida qobig'idagi } \\
\text { holatlarni o'rganish }\end{array}$} \\
\hline
\end{tabular}

Yuqoridagilardan tashqari qishloq xo'jaligi hayvonlari ekologiyasi yo'nalishi uy hayvonlarining iqlimiy omillarga javob reaktsiyalarini, oziqlanishi, yakka va poda sozlangandagi o'ziga xos xususiyatlarini o'rganadi. Keyingi yillarda "Insoniyat ekologiyasi" fani shakllanib jadal rivojlanmoqda. Shuningdek, bu fan tibbiyot, veterinariya va qishloq xo'jaligi bilan uzviy bog'liq, jumladan, etologiya hayvonlarning fe'l-atvorini o'rganadigan soha, lekin u ekologiya bilan chambarchas bog'liq. Masalan, termitlar galasi va ular o'rtasidagi munosabatlar sof etologik talqin etilsa, termitlar yashash joyi, undagi o'ziga xos sharoit ekologik tahlil etiladi.

Organizmlar tarqalishi hududiy ekologik omillariga bevosita bog'liq bo'ladi, bu jarayonni tushunib olish qator biogeografik muammolarni hal etish 
imkonini beradi.

Hayvonlar ekologiyasi sohasidagi tadqiqotlar turlarni iqlimlashtirish va ko'paytirish, qishloq-xo'jaligi zararkunandalariga qarshi kurashning ilmiy asoslangan usullarini ishlab chiqish, ovlanadigan hayvonlar sonini tartibga solish chorvachilikni rivojlantirish hamda hayvonlarni muhofaza qilishga yordam beradi.

Ekologiyaning biologiya fanlari tizimidagi o'rni va uzviyligini quyidagicha ifodalash mumkin:

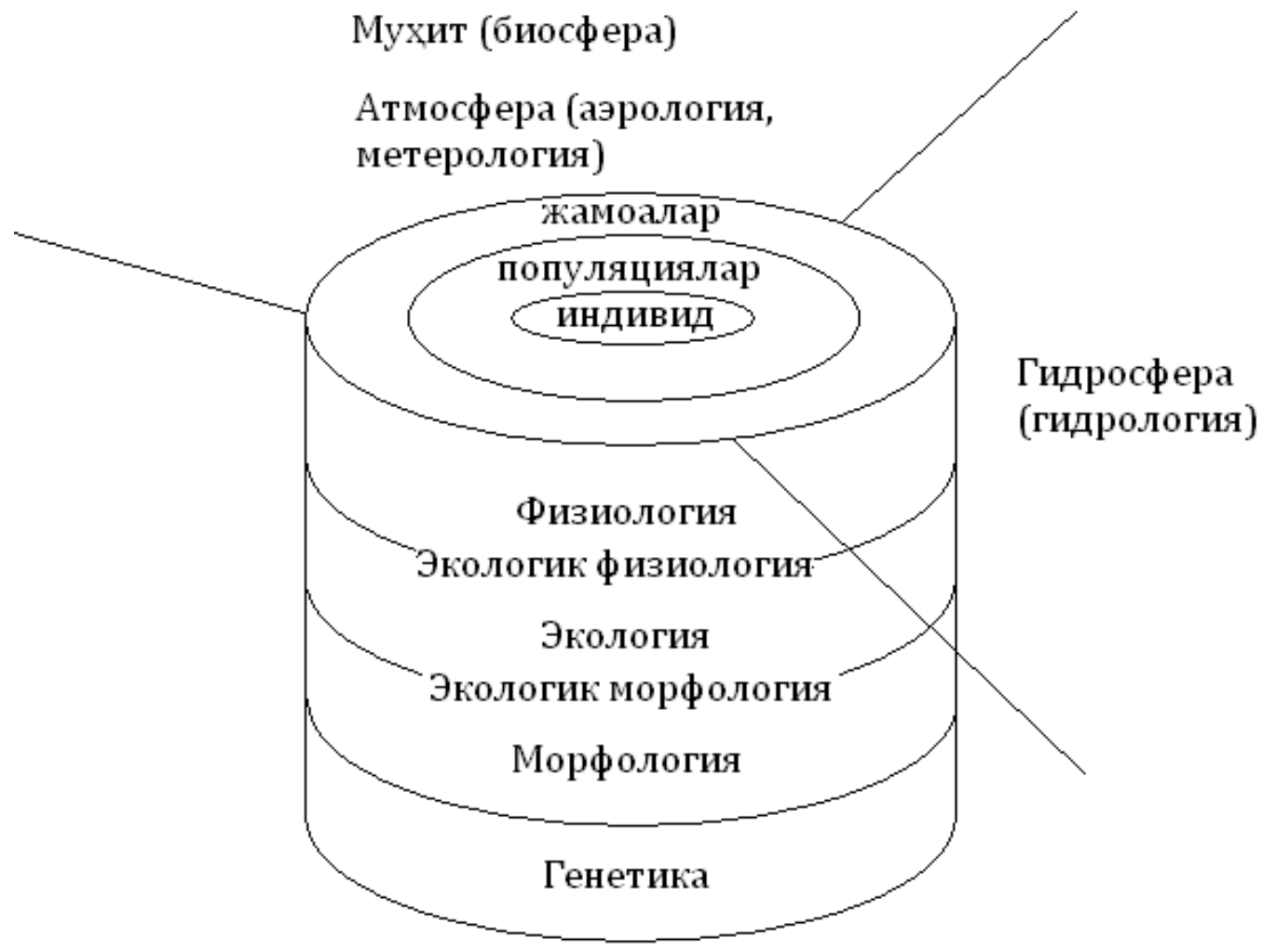

Литосфера (Геология, тупроқшунослик)

1-rasm. Ekologiyaning biologiya fanlari tizimidagi o'rni va uzviyligini

\section{Ekologiyada foydalaniladigan uslublar}

Ekologik ilmiy tadqiqotlarda kuzatish, tasviriy, taqqoslash, amaliy tajriba va modellashtirish uslublaridan foydalaniladi. Mazkur uslublar biologiya fanlarining barchasida qo'llaniladi. Ekologik ilmiy-tadqiqotlar o'ziga xosligi bilan ajralib turadi.

Tadqiqotlarda kuzatish tasviriy, qiyoslash yoki taqqoslash, amaliy tajriba va modellashtirish uslublaridan keng foydalaniladi. 
U turni alohida individ holida emas (chunki individ morfologiya, fiziologiya, sistematika uchun tadqiqot ob'ekti hisoblanadi), balki populyatsiya darajasida ham tadqiq etadi.

Populyatsiyalar muayyan hududlarni egallab, vaqt va fazoda yangilanib turadi.

Bir hayvonni turlicha tahlil qilish mumkin, fiziolog uni o'zgarmas doimiy sharoitda o'rgansa, ekolog esa aksincha, mazkur hayvonni yashash muhitida va barcha omillar majmui doirasida tadqiq etadi, zero, muhit omillari "doimiy" bo'lmay, dinamik tarzda o'zgarib turadi.

Hayvonlar ekologiyasini o'rganishda ular haqida to'liq va batafsil ma'lumot olishda eksperimental-tajribalar muhim o'rin tutadi. Ayrim hollarda laboratoriya sharoitida olingan natijalar har doim ham tabiatdagi holatni to'liq ifodalamasligi mumkin. Masalan, ayrim ignatanlilar (Echinaster sepositus) va qisqichbaqasimonlar (Eupagurus prideaauxi)ning tabiiy yashash sharoitlari mutlaq sho'r suvlar bilan bog'langan, tajribalar shuni tasdiqlaydiki, ular hatto chuchuk suvlarda ham bemalol hayot kechira olishligi kuzatilgan. Ekologik ilmiy-tadqiqotlarda dala sharoitida tabiatdagi kuzatish ishlari alohida ahamiyatga ega. Tabiatda mavjudotlar o'rtasidagi o'zaro trofik aloqalar orqali murakkab bog'lanishlar ko'pincha kuzatish - tasviriy uslubda tadqiq etiladi. Masalan, beda urug'i hosildorligi nisbiy holda mushuklarga bog'liq holda ekanligi kuzatish asosida tahlil etish mumkin. Jumladan, bedazorlar uchun pahmoq arilar alohida ahamiyatga ega, chunki beda gullari faqat ular yordamidagina changlanadi. Shu sababdan beda urug'ining salmog'i bevosita pahmoq arilarning miqdor zichligiga bog'liq. Lekin dala sichqonlari arilar uyalarini buzib ularni kamayishiga sabab bo'ladi. Mushuklar esa dala sichqonlarini qirib ushbu salbiy holatni oldini olishda muhim rol o'ynaydi.

Ushbu misol jonzotlar o'rtasida o'zaro murakkab bog'liqlik borligini isbotlash bilan birga, uni tushunish va tahlil etish faqatgina kuzatish natijalari asosida izohlash mumkinligini ko'rsatadi.

Ekologik tadqiqotlarda yuqoridagi uslublar bilan birga matematikstatistik tahlil usulidan ham foydalaniladi. Bu esa mikrotizimlarning hayoti, undagi o'zgarishlar haqida atroflicha ma'lumot olishga imkon beradi. Keyingi yillarda tabiiy jarayonlarni matematik modellashtirishga e'tibor kuchaytirilmoqda. Matematik modellar ekologik tizimlarni o'rganishda eng 
samarali natija beradi. Matematik simvollar murakkab ekologik tizimlarni ifodalashga, tenglamalar esa undagi turli komponentlarning o'zaro ta'sirini nazariy jihatdan tahlil etishga imkoniyat yaratadi.

Shuni alohida ta'kidlash lozimki, matematik model ob'ektiv borliqning to'liq bo'lmagan abstrakt in'ikosidir. Misol tariqasida sodir bo'ladigan o'zgarishlarning matematik ifodalanishini keltirish mumkin. Agar populyatsiya miqdor zichligining ortishi davomiy to'xtovsiz holat deb qaralsa, populyatsiyadagi o'sishni quyidagi eng sodda differentsial tenglama vositasida ifodalash mumkin.

\section{$D x / d t=r x$}

Bunda $t$ vaqtidagi populyatsiya zichligi- $x$; $r$-o'sishning haqiqiy tezligi, har bir tur uchun doimiy qiymat (o'zgarmas sharoitda populyatsiyadagi tug'ilish va o'limning eng yuqori tezligi). Agar $t=0$ va populyatsiya zichligi $x=0$ deb qabul qilinsa, unda ayni tenglamaning echimi quyidagi funktsiyadan iborat bo'ladi.

Bunda shu ma'lum bo'ladiki, $t$ qisqa vaqtda populyatsiyadagi ko'paygan individlar soni $r-t$ ga teng bo'ladi. Demak, bu vaqtda populyatsiyaning yosh tarkibi o'zgarmaydi. Lekin bu holat chegaralangan vaqtdagina haqiqatga yaqin bo'ladi, vaqt o'zgarishi bilan populyatsiyadagi tug'ilish va o'limning nisbati o'zgaradi, natijada ayni populyatsiyaning yosh, jinsiy tuzilmasida o'zgarish ro'y beradi.

Matematik modellar echimi asosida olingan nazariy ma'lumotlar amalda qiyosiy tahlil etiladi va nazariy hamda amaliy natijalar o'rtasidagi tafovut aniqlanadi. Matematik modellash ekologik o'zgarish va hodisalarni aniq tahlil etish, keyingi tadqiqotlar yo'nalishlarini aniqlashda etakchi omil sifatida katta ahamiyatga ega.

\section{Hayvonlar ekologiyasining qisqacha tarixi}

Insoniyat tarixining ilk davridanoq kishilar hayvonlar hayoti bilan qiziqib ularning xilma xilligiga e'tibor qaratgan Yashash sharoitlari haqidagi tasavvurlarga ega bo'lganlaridan so'ng, domestikatsiya ishlari bilan shug'ullanganlar. Qadimgi odamlarning ov qurollarini topilishi va g'orlardagi sur'atlarini o'rganish yuqoridagi fikrlarni tasdiqlaydi. 
Hayvonlar to'g'risidagi dastlabki ma'lumotlar miloddan avvalgi VI-II asrlarga oid Misr, Xitoy, Hindiston va Yunon qo'lyozmalarida uchraydi. Hind epik poemasi "Ramayana" va "Mahabxorat" rivoyatlarida 50 ga yaqin hayvonlarning yashash tarzi, fe'l-atvorlari va miqdori haqida qimmatli ma'lumotlar keltirilgan. "Bxagvata Parana" kitobida xavfli kasalliklar tarqalishida yovvoyi hayvonlarning ahamiyati yoritilgan. Antik davrda yashagan faylasuflarning ilk ilmiy asarlarida hayvonlarning turli tumanligi, xulq-atvori baliqlar, qushlarning ko'chib yurishlariga oid ma'lumotlar keltirilgan.

Zoologiya fanining asoschisi eramizdan avvalgi 384-322 yillarda yashagan yunon olimi Aristotel hisoblanadi. U o'z asarlarida 452 tur hayvonning tuzilishi, hayot kechirishi va tarqalishi to'g'risidagi ma'lumotlarni yozib qoldirgan. "Hayvonlarning paydo bo'lishi" asarida oldin zoofitlar, keyin hayvonlar rivojlangan, o'z tuzilishiga ega bo'lgandan so'ng, shaxsiy xossa namoyon bo'ladi deb ta'kidlagan.

Qadimgi rim tabiatshunosi Gay Pliniy (miloddan so'ng 23-79 yillar) "Tabiat tarixi" asarida o'sha davrda ma'lum bo'lgan barcha hayvonlarni ta'riflab, ularning ekologiyasiga oid kuzatish natijalarini ham bayon etgan. Uyg'onish davrlarigacha, jumladan, o'rta asrlarda ham zoologiya va ekologiyaning rivojlanishida aytarli siljish bo'lmadi. Faqatgina uyg'onish davridan so'ng, XV-XVI asrlarga kelib tabiiy fanlarni rivojlanishi zoologiyaga ham o'z ta'sirini ko'rsatdi. Buyuk geografik kashfiyotlar (Xristofor Kolumb, Marko Polo, Magellan va boshqalar) tufayli dunyoning turli mamlakatlarida hayvonlarga oid boy materiallar to'plandi. Shu davrda Shveytsariyalik vrach va naturalist K.Gesnerning (1516-1565) "Hayvonlar tarixi” asari paydo bo'ldi. XVII-XVIII asrlardagi ekologik ma'lumotlar mavjudotlarning ayrim guruhlarini o'rganishga yo'naltirilgan edi. Karl Linney (1707-1778) tomonidan o'simliklar dunyosining zamonaviy sistematikasiga asos solindi. J.Byuffon (17021788)ning ishlarida esa, hayvonlarning tuzilishiga tashqi muhitning ta'siri masalasi talqin qilindi. O'simlik va hayvonlarning evolyutsion o'zgarishida eng muhim omil tashqi muhit ta'siri ekanligi bilan birga J.B.Lamark (17741829)ning "Zoologiya falsafasi" asarida deistik ma'lumotlarni ham ilgari surdi.

K.F.Rule (1814-1858) hayvonlarning yashash tarzi, tashqi muhit bilan o'zaro munosabatlari va moslanish hisoblarini o'rganish zarurligini keng 
targ'ib qildi. Uning asarlarida hayvonlar hayotidagi maromlar, mavsumiy va yillik o'zgarishlar va ularning yashash tarzi, tarqalishi va boshqa ekologik xususiyatlariga oid ma'lumotlar keltirilgan. Ch.Darvin (1809-1882) "Turlarning tabiiy tanlanish yo'li bilan kelib chiqishi" asarida tabiatdagi yashash uchun kurash - tur bilan muhit o'rtasidagi har qanday qaramaqarshiliklarning ko'rinishlari tabiiy tanlanishga olib keladi deb, evolyutsiyaning harakatlantiruvchi kuchlarini (o'zgaruvchanlik, irsiyat, yashash uchun kurash va tabiiy tanlanish) xarakterlab, evolyutsion ta'limotga asos soldi.

1866 yilda nemis biologi E. Gekkel "Ekologiya” atamasini ilk bor qo'lladi, 1877 yilda esa nemis gidrobiologi K.Myobius biotsenozlar yoki tabiiy jamoalar haqidagi tushunchani izohlab berdi. XX asrning boshlarida gidrobiologiya, fitotsenologiya, botanika, zoologiya sohalarida o'ziga xos maktablar shakllandi. Shu davrdan boshlab, o'simliklar ekologiyasi, hayvonlar ekologiyasi va umumiy ekologiya mustaqil fan sifatida e'tirof etildi.

1913 yilda Ch.Adamsning "Hayvonlar ekologiyasiga oid", V.Shelfordning "Quruqlik hayvonlari jamoalari" haqidagi va S.A.Zernovning "Gidrobiologiya"ga oid maxsus jurnallari ta'sis etildi.

1930-40 yillarga kelib K.Frideriks, F.Bodengiylar va boshqalarning ekologiyaning umumiy qonuniyatlari tahliliga bag'ishlangan ishlari e'lon qilindi.

Umumiy ekologiyaning rivojlanishida D.N.Kashkarovning ilmiy tadqiqotlari alohida ahamiyatga ega. Uning "Muhit va jamoa" mavzusida Markaziy Osiyo universitetida o'qigan ma'ruzalarida, keyinchalik "Hayvonlar ekologiyasi asoslari" nomi bilan dastlabki darslik sifatida bosmadan chiqdi va "ekologiya maktabi" shakllandi.

1930-yillarda ekologiyaning yangi bir tarmog'i sifatida populyatsiyalar ekologiyasi yuzaga keldi. Ingliz ekologi Ch. Elton "Hayvonlar ekologiyasi" kitobida populyatsiya ekologiyasining asosiy yo'nalishlari, maqsad va vazifalarini ta'riflab berdi.

Populyatsiya ekologiyasiga oid tadqiqotlar ko'lamining kengayishida S.A. Severtsov, S.S. Shvarts, N.P. Naumov, G.A. Viktorov xizmatlari alohida o'rin tutadi.

Populyatsiyalarni batafsil o'rganish har bir turning biotsenozdagi o'rni 
va ahamiyatini talqin etishga imkon berdi. Ushbu yo'nalishda olib borilgan izlanishlarning samarasi sifatida "ekologik tokchalar" kontseptsiyasi yaratildi (J.Grinnel, Ch.T. Elton, R. Makartur, D.Xatchinson, G.F.Gauze).

Hayvonlarning morfologik va evolyutsion ekologiyasini rivojlanishida M.S.Gilyarov, S.S.Shvartslarning qo'shgan hissasi katta bo'ldi.

40-yillarda tabiiy ekotizmlarni o'rganishda yangi, o'ziga xos yo'nalish shakllandi. Ingliz ekologi A.Tensli (1935) ekotizim tushunchasini fanga kiritdi, V.N.Sukachev (1942) esa biogeotsenoz haqidagi ta'limotni asosladi.

50-yillarning boshlarida G.Odum, Yu.Odum, R.Uitekker, R.Margalef va boshqa olimlar biologik mahsuldorligining nazariy asoslarini yaratdilar.

Ekotizmlar va biogeotsenozlarni o'rganish borasidagi tadqiqotlarning kengayishi XX asrda biosfera haqidagi ta'limotni vujudga kelishi uchun asos bo'ldi. Bu o'rinda rus olimi V.I.Vernadskiyning xizmatlari beqiyosligi bilan ajralib turadi.

Hozirgi davrda ekologiya eng jadal rivojlanayotgan fanlardan biri, zero, u tabiatdan oqilona foydalanish va uni muhofaza qilishning nazariy asosi sifatida muhim amaliy ahamiyat kasb etadi.

\section{Markaziy Osiyo olimlarining tabiat va ekologiyaga oid fikrlari}

Tabiat bilan jamiyat o'rtasidagi ekologik bog'liqlik tarixi uzoq o'tmish bilan bog'liq. Markaziy Osiyo xalqlari, jumladan o'zbek xalqi juda qadimdan ekologik madaniyat merosiga ega.

Markaziy Osiyoning tabiati, rang-barang o'simlik va hayvonot dunyosi haqidagi ma'lumotlar qoyatosh sur'atlarida, "Avesto" kitobida, yozma yodgorliklarda va qomusiy olimlarimizning durdona asarlarida o'z ifodasini topgan. Ushbu manbalar tabiatni asrash va e'zozlash haqidagi qimmatli fikrlarni o'zida mujassamlashtirgan.

Zardo'shtiylik dinining muqaddas kitobi «Avesto»da hikmatli giyohlar, turli kasalliklarni davolashda ulardan foydalanish, tana tuzilishi, uni parvarishlash va gigienasi, issiq sharoitda murdaning buzilishi, bu esa atrofmuhitga, odamlarga xavf tug'dirishi haqida fikrlar bayon etiladi. Uning alohida boblarida go'dak, hayvon va itlarga ozor bermaslik, o'ldirmaslik, ularni asrash to'g'risida ma'lumotlar berilgan. 
Markaziy Osiyo hududida yashagan ibtidoiy odamlar ko'plab yodgorliklar qoldirganlar. G'orlar va qoyatoshlarga ular tomonidan mahorat bilan ishlangan rasmlar ushbu mintaqaning tabiati, o'simlik va hayvonot dunyosi haqidagi qadimda yashagan ajdodlarimizning tasavvurlari ancha keng qamrovli bo'lganligidan dalolat beradi. Qoyatoshlarga tushirilgan sur'atlar ichida yovvoyi ho'kiz, kiyik, tog' echkisi, arxar, ohu, to'ng'iz, bo'ri va it tasvirlari bilan bir qatorda, Markaziy Osiyoda bir vaqtlar yashagan hozirda esa uchramaydigan yoki kamayib ketgan yo'lbars, qulon, yovvoyi ot, mamontlarning tasvirlari ham bor. Bunday sur'atlar Molguzar, Nurota, Chotqol tog'lari, So'x vohasida, Afrosiyob, Shoxruhiya va Xorazmning qadimiy arxeologik ob'ektlarida ko'plab uchraydi. Mazkur ibtidoiy sarhat obidalari o'sha davrda yashagan kishilar hayoti va Markaziy Osiyo hayvonot dunyosi tarixini o'rganishda katta ahamiyatga ega.

Ajdodlarimiz qoldirgan qadimiy yozma yodgorliklar bilan tanishar ekanmiz, ular bizga tabiat hodisalari haqida batafsil ma'lumotlar qoldirganligini ko'ramiz. Qadimgi mualliflar tabiat voqea-hodisalari, o'simlik va hayvonlar hayotidagi mavsumiy o'zgarishlar, o'z davrida Markaziy Osiyo va unga qo'shni mamlakatlarda uchragan qizil bo'ri, qoplon, yo'lbars, qulon, yovvoyi ot, ohu-kiyik, arxar, tog' echkilari, to'ng'iz kabi hayvonlar haqidagi boy ma'lumotlarni yozib qoldirganlar.

O'rta asrlarda yashab ijod etgan Markaziy Osiyo allomalari Muhammad Muso al-Xorazmiy, Abu Nasr Forobiy, Abu Rayhon Abu Rayxon Beruniy, Abu Ali Ibn Sino, Zahiriddin Muhammad Bobur va boshqa olimlar o'z asarlari bilan jahon tabiatshunoslik fanlari rivojlanishiga ulkan hissa qo'shganlar.

Abu Abdullo ibn muhammad ibn Muso al-Xorazmiy (782-847) jahon matematika fanining asoschisi, tabiatshunoslik sohasida ham qomusiy bilimga ega bo'lgan olim. Uning "Er tasviri kitobi"da butun dunyo, qit’alar, okeanlar, qutblar, ekvator, sahrolar, ko'llar, o'rmonlar, barcha mamlakatlar, o'lkalar, ulardagi hayvonot va o'simlik dunyosi, tabiiy resurslari, aholi haqidagi ma'lumotlar keng o'rin olgan. Xorazmiy tabiat va odamlar bir-birini tushunishlari, tabiatdagi mutanosiblik saqlanishi lozimligini ta'kidlagan.

Abu Abdulloh Muhammad ibn Ahmad ibn Nasr Jayhoniy (870-912) yirik davlat arbobi, tabiatshunos, mashhur va bilimdon tabib sifatida shuhrat qozongan. Jayhoniy asarlarida Markaziy Osiyo, Hindiston, Xitoy, Tseylon va 
Eronning qazilma boyliklarini, o'simliklari, hayvonlari va boshqa tabiiy resurslarini batafsil ta'riflab, hayvonlar va o'simliklarning tarqalish xaritasini tuzgan. Uning Markaziy Osiyo tabiati, tabiiy resurslari va geografiyasiga oid to'plagan materiallari o'ta aniqligi va betakrorligi bilan hozirgi kunda ham o'z ahamiyatini yo'qotmagan. Jayhoniy qo'shni mintaqalarning barchasi uchun umumiy bo'lgan o'simlik va hayvonlardan tashqari alohida hududlargagina xos bo'lgan turlarning ham uchrashligini alohida ta'kidlaydi. Bu borada $\mathrm{u}$ «Endemik turlar» iborasini qo'llamagan bo'lsada, lekin ayni tushunchani ilk bor talqin etganligi shubhasiz. U turli hududlardagi yashash muhitlarini tahlil etar ekan, baland tog'li o'lkalarda havo (kislorod) siyrak bo'lishi tufayli organizmlar yashashi qiyinlashishi kabi hodisalarni ham keng sharhlaydi. Jayhoniyning yuqumli kasalliklarning tarqalish yo'llari va vositalari, bunday kasalliklarning keng tarqalib ketmasligini oldini olish haqidagi ma'lumotlari qadrlangan.

Abu Nasr Muhammad ibn Uzlug' ibn Tarxon Forobiy (873-910) o'z davrining yirik allomasi bo'lgan. Uning ilmiy-falsafiy merosi nihoyatda boyligi va sermazmunligi bilan ajralib turadi. Forobiyning tabiatshunoslikka oid «Hayvon a'zolari to'g'risida so'z», «Insoniyatning boshlanishi haqida kitob» asarlarida inson va hayvonlar organlari, ulardagi o'xshashlik va farqlar, anatomiyasi, fiziologiyasi, oilaning kelib chiqishi, odamning paydo bo'lishi va boshqa masalalarni yuritishga katta e'tibor berdi. U hayvonot dunyosini fikrlovchi, aqlli va noaql, fikrlamaydigan hayvonlardan tashkil topgan deb ta'kidlaydi. Uning talqiniga ko'ra odam organizmi yaxlit bir sistemadan iborat bo'lib, undagi jarayonlar birlamchi va ikkilamchi signallar sistemasi orqali boshqariladi. Forobiy dunyoda birinchi bo'lib organizmlardagi o'zgaruvchanlik, o'simlik va hayvon turlarining kelib chiqishida tanlanishning ahamiyatini ilmiy asoslab berdi. Uning talqiniga ko'ra, tabiatshunoslikdagi tanlanish inson yordamida va inson aralashuvisiz sodir bo'ladi, ya'ni sun'iy va tabiiy tanlanishlar bo'lishligini Ch. Darvindan oldin e'tirof etgan.

Forobiyning Evropa olimlariga qadar 1000 yil avval yaratgan ta'limoti, uning asarlari anatomiya, fiziologiya, tibbiyot va biologiyada evolyutsion qonuniyatlarni asoslashda ilmiy-nazariy dalil bo'lib xizmat qildi.

«Hudud ul-olam min al-mashriq il-mag'rib» («Sharqdan G’arbga dunyo chegarasi») asari noma'lum muallif tomonidan yozilgan va $\mathrm{X}$ asr 
tabiatshunoslik tarixida eng noyob manbalardan biri sanaladi. Mazkur asar fan tarixida qisqartirilgan «Hudud ul-olam» nomi bilan mashhurdir. «Hudud ul olam»da Er shari, qutblari, ekvator, mamlakatlar, qit'alar, ko'llar, orollar, dengizlar, sahrolar, tog'lar, daryolar, okeanlar, shaharlar, chegaralar, qo'shni mamlakatlar, ularning tabiati mufassal bayon etilgan. Muallif ayniqsa, Markaziy Osiyo o'lkalari va tabiati haqida batafsil to'xtalgan va qiziqarli materiallarni bayon etgan. Uning ta'kidlashicha, talas vodiysida samur (tiyin), sanjob (olmaxon), ohu mushruk (muskusli bug'u) ko'pdir, beshpaq dalada esa, qulonlar, yovvoyi otlar va sayg'oqlar uchraydi. Aytish joizki, bu asar Markaziy Osiyo tabiati fanlarining rivojlanishida muhim ahamiyatga molik tarixiy manba hisoblanadi.

Abu Rayhon Muhammad ibn Ahmad al-Abu Rayxon Beruniy (973-1048) buyuk o'zbek qomusiy olimi, u o'rta asrning ulkan mutafakkirlari sirasiga kiradi. Allomaning kuzatishlarida tabiatga oid ma'lumotlar mukammalligi bilan ajralib turadi. Uning asarlarida Markaziy Osiyo, Eron, Hindiston, Afg'onistonning qazilma boyliklari, dorivor o'simliklari, hayvonlari haqida aniq ma'lumotlar berilgan.

«0’tmish avlodlardan qolgan yodgorliklar» asarida Abu Rayxon Beruniy Eron shimolidagi o'simlik va hayvonlarni batafsil ta'riflaydi. Kaspiy dengizida uchrovchi qoravoy qushlarning ekologiyasiga oid o'z kuzatishlari natijalarini bayon etadi. Shu kitobda, u o'simlik va hayvonlar hayotidagi mavsumiylik va biomaromlarni keng tahlil etadi. Jumladan, u shunday yozadi: «Agar qish qattiq kelsa, yirtqich qushlar inlariga berkinadi. Bahorda ilonlar uyg'onadi, baqalar ovoz chiqaradi. Bu hodisalarning barchasi o'rtasida uzviy bog'liqlik mavjud». Abu Rayxon Beruniy o'z davrida Forobiy tomonidan talqin etilgan tabiiy va sun'iy tanlanish haqidagi fikrlarni mukammallashtirib, atroflicha kengaytiradi. Agar, deb ta'kidlaydi Abu Rayxon Beruniy, Er yuzini bir xil daraxt yoki bir xil hayvon butunlay qoplab olsa, bu holda hayvonning ko'payishiga ham, daraxt o'sishiga ham o'rin qolmaydi. Shu sababdan dehqon ekinlarni o'toq qilib, keraksizlarini yulib tashlaydi. Bog'bonlar daraxtlarga shakl beradi, keraksizlarini kesib tashlaydi. Asalari ishlamaydiganlarini yo'q qiladi. Tabiat ham, o'z yo'lida shunday ish qiladi. Abu Rayxon Beruniy fikricha, erdagi o'simlik va hayvonlar cheksiz ko'payishga intiladi, shu maqsadda kurashadi, zero, «ekin va nasl qodirish bilan dunyo to'lib boraveradi». Garchi 
dunyo cheklangan bo'lsa-da, vaqt o'tishi bilan bu ikki o'sish natijasida ko'payish cheklanmaydi. Agarda o'simliklardan yoki jonivorlardan biror xilining o'sishiga sharoit bo'lmay, o'sishdan to'xtasa ham, boshqalarida bu ahvol bo'lmaydi. Ular birdaniga paydo bo'lib, birdaniga yo'qolib ketmaydi, ularning biri yo'qolsa ham, u o'z o'xshashini qoldirib ketadi. Abu Rayxon Beruniy «Saydana» kitobida zoologiya, botanika, zoogeografiya, fitogeografiya fanlarining maqsad va vazifalarini izohlashga alohida e'tibor qaratdi.

Abu Rayxon Beruniy, yer yuzini o'zgarishi o'simlik va hayvonot dunyosining o'zgarishiga sabab bo'lishi, mumkinligini qator misollar bilan isbotlashga harakat qiladi. Abu Rayxon Beruniyning «Hindiston» asarida jirafa, karkidon, fil, kiyik, delfin va boshqa sut emizuvchilar, shuningdek, qushlarning morfologiyasi, tarqalishi va ekologiyasiga oid g'oyat qimmatli ma'lumotlar o'rin olgan.

Abu Rayxon Beruniy asarlarida hayvonlar, o'simliklar organizmning tarkibi, mazkur organizmlarni tashqi muhit bilan mustahkam bog'liqligining natijasi ekanligi ta'kidlanadi. U olimlar orasida birinchi bo'lib organik dunyoning evolyutsion taraqqiyoti, olamning oddiydan murakkabga tomon takomillashib borishi to'g'risida fikr yuritib, yashash uchun kurash va tabiiy tanlanish masalalarini asosladi. Shuning bilan birga, Er o'z o'qi va Quyosh atrofida aylanadi deb geliotsentrik ta'limotni ilgari surgan.

Abu Ali ibn Sino (980-1097)ning falsafiy va tabiiy-ilmiy qarashlari uning mashhur "Kitob ash-shifo" ("Davolash kitobi") asarida mujassamlashgan. Mazkur asarda kishilar organizmiga tashqi muhitning ta'siri, uning ahamiyati, kasalliklarining tarqalishida suv, havo va shamolning ahamiyati bayon etilgan. Shuningdek, asarda odamlarning ichagida parazitlik qiluvchi chuvalchanglar keltirib chiqaradigan kasalliklar va ularni davolash to'g'risida, hamda chuvalchanglar turlari haqida ham qator ma'lumotlar berilgan. Uning “Tib qonunlari" shox asari tibbiyot ilmining qomusi hisoblanadi.

Zahiriddin Muhammad Bobur (1483-1530)ning shoh asari «Boburnoma»da Markaziy Osiyo, Afg'oniston mamlakatlarining tarixi, tabiati, etnografiyasi, geografiyasi va tibbiyotiga oid boy materiallar o'rin olgan. Bobur Toshkent, Farg'ona vodiysi, Afg'oniston va Hindiston hayvonlarini mohirona ta'riflagan. U Hindistonda uchrovchi 60 dan ortiq umurtqali hayvonlarni batafsil bayon etadi va mazkur hayvonlarni yashash muhitiga 
qarab 4 guruhga ajratadi: daraxtda yashovchi hayvonlar, quruqlik hayvonlari, suv yaqinida uchraydigan hayvonlar va suv hayvonlari.

Boburning fikricha, Hindistonning o'simlik va hayvonlari o'ziga xos endemikdir. Mazkur mintaqa hayvonlarining rangi qora yoki qoramtir, hatto ularning ko'zi ham qora, ehtimol bu Hindistonning geografik joylanishi va iqlimiy sharoitlariga bog'liqdir. Hindiston hayvonlari shu xususiyatlari bilan Markaziy Osiyo faunasidan keskin farq qiladi.

\section{O'zbekistonda hayvonlar ekologiyasi fanining qisqacha rivojlanish tarixi}

O'zbekistonning hayvonot dunyosini o'rganish uzoq muddat davom etgan uzilishdan so'ng, XIX asrning ikkinchi yarmida qaytadan boshlandi. Bu boradagi dastlabki tadqiqotlar N.A.Severtsov, A.P.Fedchenko va V.F.Oshaninlar nomi bilan bog'liq.

N.A.Severtsov (1827-1885) o'z sayohatlarida Orol dengizi, Ustyurt, Qizilqumning shimoliy hududlari, Sirdaryo, Tyonshon va Pomir tog'lari tabiati va hayvonot dunyosini o'rganishga oid qimmatli ma'lumotlarni yig'di. Uning tadqiqotlari natijalari aks etgan "Turkiston hayvonlarining vertikal va gorizontal tarqalishi" asari o'z qimmati bilan muhim ahamiyat kasb etadi. 1868-1871 yillarda Oloy va Zarafshonga katta ekspeditsiya safari uyushtirildi. Unda faoliyat ko'rsatgan A.P.Fedchenko mazkur mintaqada uchrovchi rishtaning bioekologik xususiyatini o'rgandi. V.F.Oshanin (1844-1945) esa Oloy vodiysi, Zarafshon, Turkiston va Pomir tog'lari tabiatini tekshirdi, Amudaryo yuqori havzasi hasharotlarini tadqiq etdi. U o'zining asarlarida hasharotlarning 700 dan ortiq, birgina saratonlarning 200 dan ortiq turi haqida ma'lumotni taqdim etdi.

XX asrning 20-yillaridan boshlab sobiq O'rta Osiyo hozirgi O'zbekiston Milliy universiteti ekologik tadqiqotlar markaziga aylandi.

A.L.Brodskiy (1882-1943) Qizilqum faunasini o'rganib, chig'anoqli soxta oyoqlilarning bir qancha yangi turlarini topdi. Qizilqum cho'li soxta oyoqlilarini dengiz soxta oyoqlilari bilan morfologik bir muncha yaqinligini isbotlab, Markaziy Osiyo faunasining genezisi haqidagi fikrlarini bildirdi.

D.N.Kashkarov (1878-1941) esa O’zbekistonda keng ma'nodagi ekologik 
tadqiqotlarni boshlab, o'zining ekologik maktabini shakllantirdi. Olim kemiruvchi hayvonlarning biologiyasi, sistematikasini o'rganish bilan birga, hayvonlar ekologiyasi bo'yicha ham qator ilmiy izlanishlar olib bordi. Natijada, u birinchilardan bo'lib Markaziy Osiyoni zooekologik tavsifladi, cho'l biotsenozi va undagi trofik aloqalarni talqin etdi. Uning asarlarida turli landshaftlarning tuzilmalari, biotsenozlarning ozuqa zanjiri, tuzilmalari va ulardagi trofik aloqalar, shuningdek, landshaftlar genezisi va evolyutsiyasiga oid ilmiy ma'lumotlar tahlil etilgan omillar majmuasi, ular ta'sirida landshaftlarning o'zgarishi, ayniqsa bu jarayonga antropik omil ta'siri kuchli ekanligi D.N.Kashkarov ishlarida alohida ta'kidlanadi. Uning tadqiqotlari asosida tabiatni muhofaza qilish, undan oqilona foydalanish muammolarning qator nazariy va amaliy masalalari o'z echimini topdi.

O’zbekistonda ekologik yo'nalishdagi ishlarning asoschilari D.N.Kashkarov va E.P.Korovinlar hisoblanib, ularning hamkorlikdagi ilmiy faoliyati mahsuli sifatida ekologiya va geografiya maktablari shakllandi. Mazkur maktab sobiq Ittifoq miqyosida ham etakchi va alohida mavqega ega bo'lib, ekologiya fanining taraqqiyotidagi o'rni beqiyos bo'ldi.

1930 yillar davomida D.N.Kashkarov va E.P.Korovinlar tomonidan "Cho'ldagi hayot", "Muhit va jamoa", "Markaziy Osiyo va Qozog'iston cho'llarining turlari va ulardan xo'jalikda foydalanish istiqbollari", "Uy hayvonlari ekologiyasi" va boshqa ko'plab ilmiy asarlar chop etildi. Ushbu asarlarda ekologiya fanining maqsad va vazifalari, tadqiqot uslublari, organizm va muhit o'rtasidagi munosabatlar, organizmlarning yashash muhitiga moslanish xususiyatlari, shuningdek ekologiyaning boshqa dolzarb muammolari talqin etilgan.

1920-40 yillarda O'zbekistonda ekologik tadqiqotlar jadal rivojlana bordi. Bu borada D.N. Kashkarov izdoshlari V.A. Selevin, T.Z. Zoxidov, I.I. Kolesnikovlarning faoliyati samarali bo'ldi.

1950 yilda O'zbekiston Fanlar Akademiyasining Zoologiya va parazitologiya instituti tashkil etildi. Yangi ilmiy-tadqiqot markazi oldiga O'zbekiston hayvonot dunyosini o'rganish, muxofaza qilish va ulardan oqilona foydalanish muammolari echimini topishga qaratilgan batafsil tadqiqotlar olib borish vazifasi qo'yildi. O'zbekistonda zoologik - ekologik tadqiqotlarning samarali rivojlanishida T.Z.Zoxidov, A.M.Muxammadiev, V.V.Yaxontov, 
M.A.Sultonov, R.O.Olimjonov, A.T.To'laganov kabi olimlar o'zlarining munosib hissalarini qo'shdilar.

Zooekologik yo'nalishdagi keng qamrovli ishlarning rivojlanishi T.Z.Zoxidov nomi bilan bog'langan. Olim 1971 yilda Qizilqum faunasini o'rganish bo'yicha olib borgan tadqiqotlari asosida “Qizilqum cho'lining biotsenozlari" monografiyasini yaratdi. Muallif o'z asarida Qizilqum cho'llarining o'ziga xos hayot makoni ekanligini ta'riflab, undagi qumli, sho'rhok, loyli va toshloqli cho'llarni mustaqil biotoplarga ajratadi. Har bir biotop mayda hududiy birliklar - fastsiyalardan tashkil topishi mumkinligini ta'kidlaydi. T.Z.Zoxidov talqiniga ko'ra, har bir mintaqa, undagi biotop va fastsiyalar alohida ekologik faunistik tahlil etilishi va mintaqaviy rayonlashtirishda bu holatni inobatga olish zarur.

V.V.Yaxontovning "Hasharotlar ekologiyasi" (1963) nomli risolasi mazmunli darslik sifatida hozirgi kunda ham o'z ahamiyatini yo'qotmagan.

M.A.Sultonovning “O'zbekiston qushlarining gelmintlari” (1963), A.M.Muxammadievning "Suv havzalari gidrobiologiyasi" sohaga oid ilmiy tadqiqot ishlarining kengayishida muhim rol o'ynaydi. 1970-80 yillarda O'zbekistonda entomologik (R.O.Olimjonov, S.N.Alimuxammedov, M.N.Narziqulov, A.G.Davletshina va ularning shogirdlari), gelmintologik (A.T.To'laganov, M.A.Sultonov, I.X.Ergashev, J.A.Azimov va boshqalar) va gidrobiologik-ixtiologik (A.M.Muxammadiev, G'.K.Komilov va boshqalarning) tadqiqotlari etakchi o'rinni egalladi.

Keyingi yillarda O'zbekiston zoologlari "Hayvonot olamini qo'riqlash va ulardan oqilona foydalanish" dasturi bo'yicha "Ekologik xilma-xillikni saqlash milliy strategiya va harakat rejasi" doirasida tadqiqotlar olib bormoqdalar. Mazkur dasturlarga muvofiq O'zbekiston Respublikasi Fanlar akademiyasining zoologiya instituti, oliy o'quv yurtlari va boshqa ilmiy tadqiqot institutlarida olib borilayotgan zoologo-ekologik izlanishlar akademik J.A.Azimov rahbarligida yagona tizimga keltirilib, aniq maqsadga yo'naltirilgan.

\section{Hayvonlar ekologiyasi fanining vazifalari}

Hayvonlar ekologiyasi fani har bir tarmoq yo'nalishida muayyan 
maqsadga yo'naltirilgan muammolar echimini topishi zarur.

Hayvonlar ekologiyasi fanining amaliy vazifasi, eng avvalo, O'zbekiston hayvonot dunyosini o'rganish, ulardan va biologik xilma-xillikni saqlash borasidagi umumbashariy, mintaqaviy va mahalliy muammolarni hal etishda o'z ifodasini topadi.

Chorvachilikda qishloq xo'jalik hayvonlarini sermahsul zotlarini yaratish va iqlimlashtirish, ularni turli kasalliklardan himoya qilishning ilmiy asoslarini ishlab chiqish, chorvachilik maxsulotlariga bo'lgan inson ehtiyojlarini to'laroq qondirish borasidagi tadqiqotlarni kengaytirish zarur.

Yovvoyi va uy hayvonlarining tabiiy biotsenozlarning ta'sirini o'rganish, biotsenozlar mahsuldorligi va barqarorligini saqlash eng dolzarb vazifalardan sanaladi.

Qishloq xo'jalik zararkunandalarining tur tarkibini, ularning turli mintaqalar sharoitidagi ekologik xususiyatlarini qiyosiy tahlil etish, zararkunandalar populyatsiya zichliklarining mavsumiy va ko'p yillik o'zgarishlarini o'rganish, populyatsiya sur'atlari o'zgarishdagi o'ziga xos davriylik qonuniyatlarini o'rganish asosida yillik va kelajak bashoratini, ularga qarshi atrof-muhit va insonlar uchun xavfsiz kurash choralarini ishlab chiqish, biologik kurash usulini keng joriy etish amaliy ekologiyaning kundalik vazifalaridandir.

Baliqchilikni rivojlantirishda baliqlarning rivojlanishi, o'sishi va davriy ko'chishlarini o'rganish, suv havzalari va ayniqsa, hovuz baliqchiligi mahsuldorligini oshirishning ekologik echimini topish va amaliyotga tadbiq etish zarur.

Ovchilikni xo'jalik sohasiga o'tish, ovlanadigan hayvonlarning tabiiy zahiralarini o'rganish, miqdor zichliklaridagi mavsumiy va yillik o'zgarishlarni aniqlash asosida, rejali ovlashga oid ekologik qoidalarni ishlab chiqish hamda, ularni amalga oshirish taqozo etiladi.

Biologik xilma-xillikni saqlash va boyitish maqsadida ayrim turlarni iqlimlashtirish masalalarini hal etish zarur.

Tibbiyot va veterinariya zoologiyasi (ekologiyasi) oldida turli kasalliklarni keltirib chiqaruvchi hayvonlarni o'rganish va ularga qarshi samarali kurash usullarini ishlab chiqish vazifasi turadi.

Keyingi yillarda atrof-muhitning texnogen va geokimyoviy ifloslanishi 
darajasini bioindikatsiyasiga alohida e'tibor berilmoqda. Unga muvofiq atrofmuhit ifloslanishiga o'ta sezgir hayvon turlarini aniqlash, ularda ro'y bergan morfologik, fiziologik va ekologik o'zgarishlar asosida ifloslanish darajasini tahlil etish, bashorat qilish qonuniyatlarini ishlab chiqish hozirgi kunning eng dolzarb muammolari sirasiga kiradi. Yangi erlarni o'zlashtirish antropogen ta'sir natijasida faunaning o'zgarishi, agrotsenozlarni tiklanishi va shakllanishi xususiyatlarini tadqiq etish asosida ushbu masalalarning nazariy va amaliy echimlarini topish taqozo etiladi. 


\section{2-BO'LIM. AUTOEKOLOGIYA.}

\section{HAYVONLARNING YASHASH MUHITLARI VA ADAPTATSIYALAR.}

Hayvonlarning yashash muhitlari hilma-xil bo'lib, undagi shartsharoitlar o'ziga xos va o'zgaruvchanligi bilan ajralib turadi. Barcha mavjudotlar kabi hayvonlar ham nafaqat o'zaro, balki muhit omillari bilan doimiy mustahkam aloqada bo'ladi. Hayvonlar hayoti davomida muhitning o'zgaruvchan sharoitlariga doimo moslashib boradi, va o'zining hayotiy jarayonlarini muvofiqlashtiradi.

Hayvonlar shart-sharoitlari bilan keskin farqlanadigan to'rt xil yashash muhitida tarqalgan. Ular dastlab suvda, keyinchalik quruqlik va havoda yashashga moslashgan, sekin asta tuproqni ham egallagan, ma'lum bir guruhlari esa, organizm tanasida parazitlikka yoki simbiotik hayot kechirishga ixtisoslashgan.

Hayvonlarning muhit omillariga moslanishi ularning adaptatsiyasi deb nomlanadi. Adaptatsiya tiriklik uchun xos bo'lgan murakkab xususiyatlardan biri. Uning mahsuli sifatida hayvonlarning yashovchanligi ko'payishi va rivojlanishlari turli darajada yuzalanadi. Adaptatsiya hujayra darajasidan boshlab, hayvonlarning fe'l-atvorida, jamoalar, ekosistemalarning shakllanishi va barqarorligida o'z ifodasini topadi. Evolyutsiya jarayonida organizmlar adaptatsiya xususiyatlari yuzalanibgina qolmay, balki u o'zgarib ham boradi.

Organizmlarga ta'sir etuvchi muhitning bir qismi yoki xususiyati ekologik omillar deb nomlanadi. Ekologik omillar turlicha bo'lishi va ular organizmlarga ijobiy yoki salbiy ta'sir etishi mumkin. Ularga,

\section{Abiotik}

2. Biotik

3. Antropogen omillarni kiritish mumkin.

Abiotik omillarga yorug'lik, harorat, namlik, iqlim joyining rel'efi, tuproqning mexanik, kimyoviy va fizik xossalari, radioaktiv nurlanish, bosim, suvning sho'rlanganligi, shamol va oqim kiradi. Bularning barchasi hayvonlarga to'g'ridan-to'g'ri yoki bevosita ta'sir etadi.

Biotik omillar barcha mavjudotlar o'rtasidagi murakkab munosabatlar va o'zaro aloqalarning majmuidir (mutualizm, parazitizm, kommensalizm va 
boshq.). Har bir oranizmga boshqa mavjudotlar bevosita va bilvosita o'z ta'sirini o'tkazadi. Organizmlar o'rtasidagi biotik munosabatlar biotsenoz va populyatsiyalarning barqarorligini ta'minlovchi eng asosiy omil hisoblanadi.

Antropogen omil - inson faoliyatining barcha yo'nalishidagi ko'rinishi. Insoniyat ijtimoiy-tarixiy taraqqiyoti davomida tabiatga o'zining ijobiy yoki salbiy ta'sirini o'tkazib kelgan. Bu jarayon keyingi yuz yillikda kuchayib borib, tabiatning butunlay o'zgarishiga sabab bo'ldi. Hozirgi kunda zamin tabiati, o'simlik va hayvonot dunyosining taqdiri kishilik jamiyati - antropik omil faoliyatiga bog'lanib qolgan.

Alohida olingan omilning har biri turli hayvonlarga o'ziga xos ta'sir ko'rsatadi. Masalan, kuchli shamol ochiqlikda yashovchi yirik hayvonlarga noqulaylik tug'dirsa-da, lekin uning kuchi uyalarida yashirinib yotgan mayda jonivorlar uchun umuman sezilarsiz bo'lishi mumkin.

Qator omillar uzoq davr mobaynida o'zgarmaslik xususiyatiga ega. Jumladan, erning tortishish kuchi, quyosh, dengiz va okeanlarning tuz tarkibi, atmosfera xossasi turlarning uzoq muddatli tarixiy-evolyutsion jarayonida ham nisbatan doimiylik xususiyatini saqlab qolgan. Harorat, namlik, yorug'lik kuchi, yong'inlar, shamol kabi omillar esa vaqt va fazoda o'zgarishda bo'ladi. Har bir omilning o'zgaruvchanlik darajasi hayvonlarning yashash muhitiga bog'liq. Masalan; harorat kuchi quruqlikda o'zgarib tursada, okean va dengizlar tubi, g'orlarda deyarli o'zgarmaydi. Parazitlar uchun xo'jayin organizmi kafolatli ozuqa manba hisoblansa, yirtqichlar hayoti, aksariyat hollarda miqdor zichligiga bog'liq bo'ladi. Muhit sutkalik, mavsumiy obhavoning o'zgaruvchanligi, shuningdek, tabiiy ofatlar - bo'ron, er silkinishlari va siljishlari, suv toshqinlari natijasida muhit omillari kutilmagan holda tartibsiz o'zgaradi.

Muhit omillari ma'lum vaqt davomida, ba'zida uzoq davr mobaynida ma'lum yo'nalishdagi o'zgarishda bo'ladi. Jumladan, keyingi yillarda atmosfera tarkibidagi karbonat angidrid ulushi ortib bormoqda, natijada Er yuzasining harorati 100 yil ichida $0,5-1^{\circ} \mathrm{C}$ ga ortdi. Ushbu holat shu zaylda davom etsa, XX asrga kelib, bu ko'rsatkich yana $1,5-4,5^{\circ} \mathrm{S}$ ga ko'tarilishi mumkin. Muhit omillari hayvonlarga qo'zg'atuvchi, chegaralovchi, muvofiqlashtiruvchi va ogohlantiruvchi kuch sifatida o'z ta'sirini o'tkazadi. Muhit natijasida hayvonlar organizmida borayotgan fiziologik va biokimyoviy jarayonlar sharoitga mos 
ravishda o'zgarib bir maromda mutanosiblashadi. Omillarning chegaralovchanlik kuchi ayni sharoitda hayvonlar yashashi uchun noqulay vaziyatni yuzaga keltiradi. Hayvonlardagi anatomik va morfologik moslanishlar muhit omillarining muvofiqlashtiruvchi modifikatsiyalovchi ta'sirining mahsulidir. U yoki bu omil signal sifatida boshqa omillarning o'zgarishidan darak beradi, ogohlantiradi.

\section{Muhit omillarining ta'sir etishini umumiy qonuniyatlari}

Ekologik omillar qanchalik xilma-xil va ularning organizmlariga ta'sir etish yo'nalishlari turlicha bo'lishiga qaramay muhit omillarining mavjudotlarga ta'sir etish nuqtai nazaridan ushbu jarayonlarning umumiy qonuniyatlari farqlanadi.

Optimum qonuni. Har qanday omilning mavjudotlarga bo'lgan ijobiy yoki salbiy ta'siri, uning kuchiga bog'liq bo'ladi. Omilning mavjudot yashashi va rivojlanishi uchun eng qulay miqdor-kuchi optimum chegarasi yoki normal hayot zonasi deb nomlanadi. Optimumdan qanchalik chetga chiqila boshlansa, tur uchun xos bo'lgan yuqori va quyi kritik nuqtalari ta'sir ko'rsata boshlaydi. Omil jadallashuvini ushbu nuqtalardan chetga chiqishi natijasida mavjudot nobud bo'ladi (2-rasm).

Kritik nuqtalar chegarasi turning yashovchanlik va moslanuvchanlik darajasini belgilab, uning ekologik valentligini ifodalaydi. Turlar optimum talab darajasi va ekologik valentligi bilan bir-biridan farqlanadi. Masalan, tundradagi oq tulkilar havo haroratining $80^{\circ} \mathrm{S}$ ga $\left(\mathrm{Q} 30^{\circ} \mathrm{S}\right.$ dan $-55^{\circ} \mathrm{S}$ gacha) qadar bo'lgan o'zgarishlariga moslashgan.

Issiq buloqlarda yashovchi Copilia mirabilis qisqichbaqasi suv haroratining $6^{\circ} \mathrm{S}\left(23^{\circ} \mathrm{S}\right.$ dan $2^{\circ} \mathrm{S}$ ga qadar) chegarasidagi o'zgarishlarga chidaydi xolos.

Keng ekologik valentlikka ega bo'lgan hayvonlar nomlanishiga "evri" old qo'shimchasi qo'yib aytiladi. Masalan, evriterm, evribiont, evrigidrid, evrigal, evribatlar va boshqalar. Aksincha past ekologik valentlikka ega bo'lganlariga "steno" old qo'shimchasi qo'llaniladi, jumladan: stenobiontlar, stenoterm, stenobat, stenogidrid, stenogallar.

Ekologik omilning jadalashuviga bog'liq holda turning chidamlilik 
chegarasini ifodalovchi sxema quyida ifodalandi.

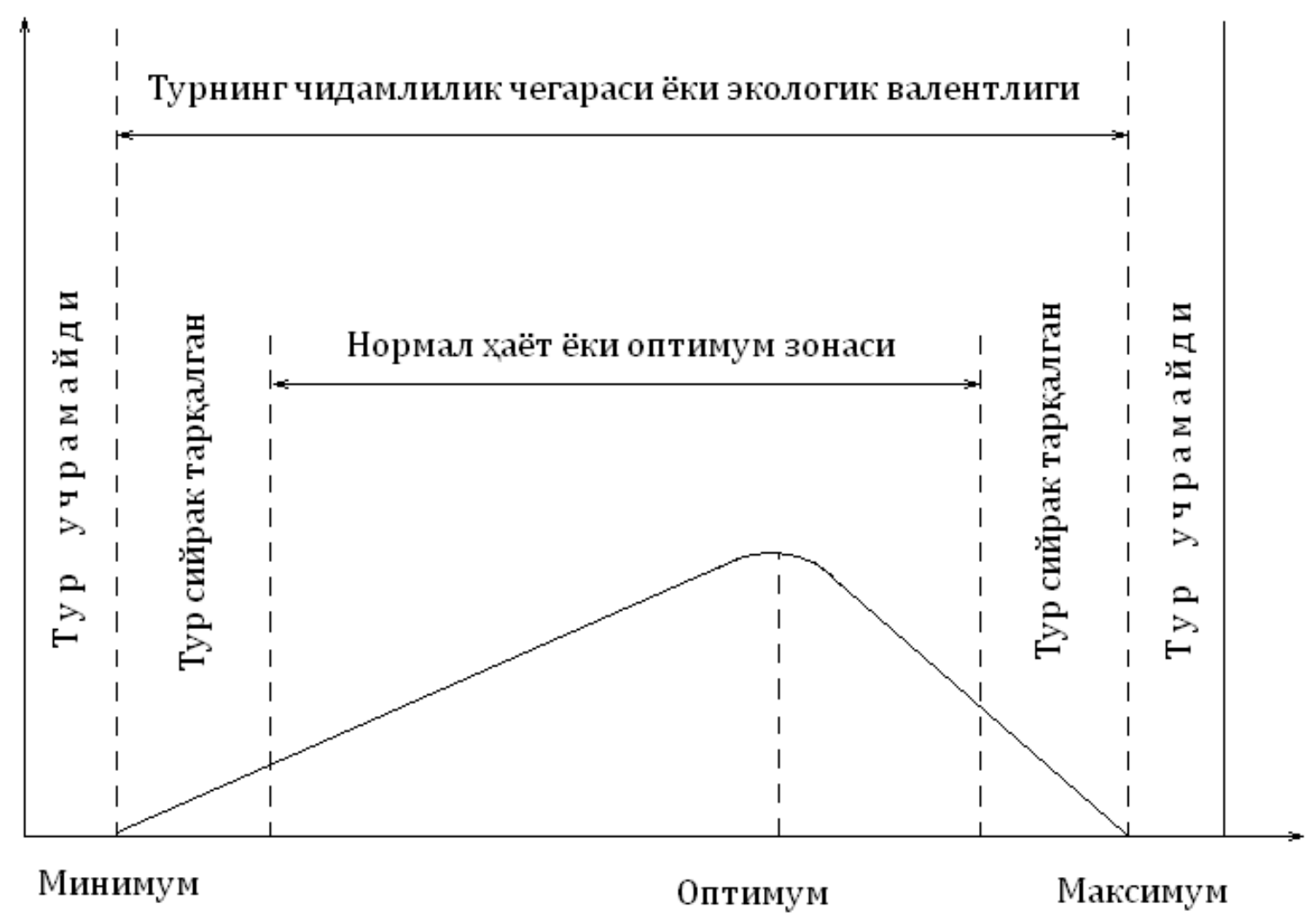

2-rasm. Ekologik omilning jadallashuvi

Turning valentligi ekologik sharoitlari o'zgaruvchan yashash muhitlarini ishg'ol eta olish darajasini ham belgilaydi. Past ekologik valentlikka ega bo'lgan turlar omillarning o'ta chegaralangan darajadagi o'zgarishlariga moslashgan bo'ladi va ularni stenotoplar deb nomlanadi. Agar tur xilma-xil va o'zgaruvchan ekologik sharoitda yashashga moslashgan bo'lsa, ulardagi ekologik valentlik chegarasi bir muncha kengligini bildiradi va bunday hayvonlar evritoplar guruhini tashkil etadi.

Ekologik omillarni hayvonlarning turli funktsiyalariga bir xil ta'sir etmasligi qoidasi. Har bir omil hayvonlarning u yoki bu funktsiyalariga turlicha ta'sir etadi. Muayyan bir hayotiy jarayon uchun optimum bo'lgan omil kuchi boshqasi uchun pessimum bo'lishi mumkin. Masalan, Q40-4505 li harorat sovuqqonli hayvonlardagi modda almashinuvi jarayonini tezlashtirsada, lekin ulardagi faollikni keskin pasaytiradi. Bunda hayvonlar tinim holatiga o'tadi. Baliqlar tuxumlarini (uvildirig'ini) etilishi uchun muvofiq bo'lgan harorat uni tashlash uchun etarli bo'lmasligi mumkin. 


\section{Muhit omillari ta'siriga tur individlari javob reaktsiyalarining}

o'zgaruvchanligi va xilma-xilligi qoidasi. Tur individlarining muhit omillariga optimal va pessimal chegaralari o'ziga xosligi bilan ajralib turadi. $\mathrm{Bu}$ farqlar individlarning nasliy, jinsiy, yosh, fiziologik xususiyatlari bilan bog'liq bo'lishi mumkin. Don mahsulotlari zararkunandasi - parvona kapalagi qurti uchun minimal kritik harorat $-7^{0} \mathrm{~S}$, etuklari uchun $-10^{0} \mathrm{~S}$ da qurtlar nobud bo'lsa-da, lekin zararkunandaning kapalaklari va tuxumlari omon qoladi.

Ko'sak qurtiga qarshi qo'llanilgan kimyoviy zaharlar g'o'za tunlami kapalagining tuxumlariga deyarli ta'sir etmaydi.

Har bir omilga hayvonlarning nisbiy mustaqil moslanish qoidasi. Muhitning xar bir omiliga nisbatan hayvonlarning ekologik valentligi o'ziga xos bo'ladi. Biror omilga chidamli bo'lgan hayvon boshqa omillarga ham bir xil darajada bardoshli bo'lmasligi mumkin. Masalan, cho'l va tog' oldi mintaqalarida uchrovchi hasharotlar quruq va issiq iqlimga moslashgan, ular yuqori namlik sharoitida yashay olmaydi.

Turlar ekologik spektrining o'ziga xosligi qoidasi. Tashqi muhitning barcha omillariga nisbatan bo'lgan talabi - ekologik spektri o'ziga xos bo'lib, hatto bir xil sharoitda yashaydigan turlarning "ekologik spektri" bir-biridan farq qiladi.

Omillarning o'zaro ta'sir etish qoidasi. Omilning hayvonlarga bo'lgan ijobiy yoki salbiy ta'siri uning jadallik darajasiga, shuningdek bir vaqtning o'zida ta'sir etayotgan boshqa omillarga ham bog'liq bo'ladi. Hayvonlar uchun optimal bo'lgan omil boshqa omillar ta'sirida salbiy tomonga o'zgarishi mumkin. Masalan, hayvonlarga yuqori haroratning namlik bilan bo'lgan ta'siri kuchli bo'ladi. Past haroratning ta'sir kuchi shamol bilan yanada ortadi.

Cheklovchi omillar qoidasi. Muhit omillari hayvonlar uchun qulay sharoitni yaratish bilan birga, ularning yashashini cheklab ham qo'yish mumkin. Turning ekologik spektridagi birgina omilni kritik chegaradan chiqishi uni nobud qiladi. Cheklovchi omillar turning arealini belgilaydi. Turlarning shimolga tomon siljishiga past harorat ta'sir etsa, qurg'oqchil va cho'l mintaqalarida esa namlik va yuqori haroratning ta'siri cheklovchi hisoblanadi. Sebarganing changlanishi va urug' berishi paxmoq arilarga bog'liq bo'ladi. Dengiz suvi tarkibidagi fosfatlar miqdori ham cheklovchi 
ahamiyatga ega. Uni me'yorida bo'lishi planktonlarning mo'lligi va mahsuldorligini belgilaydi.

\section{Abiotik omillar va ularga hayvonlarning moslanish xususiyatlari}

Populyasiyalardagi, demak, tur ichidagi individlarning o'zaro munosabati xilma-xil, murakkab va ziddiyatli bo'ladi. Pirovard natijada ular populyasiya va turning saqlanib kolishiga xizmat kiladi. Butun populyasiya uchun foydali bo'lgan turga xos moslanishlar ayrim individlar uchun zararli va ularning nobud bo'lishiga sabab bo'lishi mumkin. Masalan, baliqchi qushlar bolalari juda ko'payib ketganida ularning bir qismini yo'qotadi, ya'ni yeb qo'yadi yoki boshiga urib, yegan ozig'ini qaytarib chiqarishga majbur etadi, shunda chiqarib tashlangan oziqni tezda voyaga yetgan qushlar yeb ketadi. Ba'zan chittaklar 1-2 ta bolasini uyasidagi to'shamaga bosib o'ldiradi.

Bir populyasiya individlari bir-biri bilan bevosita to'qnash kelmasligiga yordam beradigan bir qancha moslanishlar bor. Masalan, qo'ng'ir ayiq daraxtlarni tirnab o'ziga oziq topadigan joy chegarasini belgilaydi. Bo'ri o'zi oziqlanadigan maydonni siydigi bilan belgilaydi. Chittak, qizilto'shning erkaklari sayrab, o'zi oziqlanadigan joynn ma'lum qilib turadi. Hayvonlar boshqalar joyining chegarasini buzishdan ehtiyot bo'ladi. Bir turdagi hayvonlar populyasiyasida o'zaro yordam va hamkorlik mavjudligini kuzatish mumkin: naslni birgalashib boqish, tarbiyalash va asrash shular jumlasidandir (masalan, asalari oilalarida, yilqilar podasida). Shunday kilib, tur ichidagi kurash tur individlari serpushtligining kamayishi va bir qismining nobud bo'lishi bilan birga davom etib boradi. Biroq, bu - umuman olganda, turning yashash muxitiga, shu kurashni keltirib chiqaradigan faktorlarga ko'proq moslanish tomonga qarab avlodlar davomida takomillashib borishiga yordam beradi.

Moslashganlikning xilma-xilligi. Hayvonlarning tashqi va ichki tuzilishi, instinktlari, xatti-harakati xayot sharoitiga moslashib borishi bilan 
xarakterlanadi. Bir muhitning o'zida har xil hayvonlar turlicha moslashgan bo'ladi. Krot oyoqlari bilan yerni kovlaydi, ko'rsichqon esa boshi va kuchli kurak tishlari bilan yer tagidan yo'l ochadi. Tyulen kurak oyoqlari, delfin esa dum suzgich kanotlari yordamida suzadi. Har xil moslamalari borligi uchun o'simliklar chetdan ham changlanadi. Hujayra shirasida konsentrlangan qand eritmasi borligi tufayli qarg'atuyoq bilan ko'k pereleskaning bahorgi nozik nihollari noldan past temperaturaga ham chidaydi. Daraxt va butalarning past bo'yli va mayda bargli bo'lishi, ildizlarining yuza joylashishi, bahor bilan yozda o'simliklarning juda tez rivojlanishi, bularning hammasida tundrada hayot kechirishga moslashishning ta'siri bor. Ko'payish intensivligining har xil bo'lishi tur, uning populyasiyalari saklanishiga xizmat qiladigan muhim moslanishdir. Nasli ko'plab nobud bo'ladigan turlar (parazit chuvalchanglar, baliqlarning ko'p turlari) eng ko'p sonli nasl bersa, nasl uchun qayg'urish instinkti rivojlangan turlar kam sonli nasl beradigan bo'ladi. Kolyushka degan mayda baliq erkagi quradigan uyasiga atigi 120-150 dona uvildiriq tashlaydi, urug'langan uvildiriq va undan chiqqan chavaq baliqlarni erkagi qo'riqlaydi. Treska degan baliq esa $4 \mathrm{mln}$ ga yakin uvildiriq tashlaydi, lekin naslini qo'riqlamaydi.

Organizmlarda moslanishlar paydo bo'lishi. Atrof-muhitning konkret sharoitiga murakkab va xilma-xil moslanishlar paydo bo'lishini Darvin nazariyasi materialistik nuqtai nazardan tushuntirib beradi. Yashil barglarda yashaydigan biror populyasiyaga mansub qurtlar tanasining himoya rangi qanday paydo bo'lganini ko'rib chiqamiz. Ularning ajdodlari boshqa rangda va barglar bilan oziqlanmagan bo'lishi mumkin. Qandaydir biror hodisa tufayli ular yashil barglar bilan oziqlanishga majbur bo'lgan, deb faraz qilaylik. Doim ro'y berib turadigan mutasiyalar tufayli bu hasharotlar populyasiyalarining rangi bir xil bo'lmagan, shuning uchun juda ko'p kurtlar ichida barglar orasida kamrok ko'zga tashlanadigan och yashil tusli individlar ham bo'lgan. Yashash uchun kurashda tabiiy tanlanish ta'sirida asosan och 
yashil tusli individlar yashab qolgan va nasl qoldirgan. Keyingi avlodlarda bu prosess davom etgan, qurtlar tanasining rangi atrof-muhitning asosiy rangiga tobora ko'proq moslashib borgan. Mimikriya hosil bo'lishini ham xuddi shunday tushuntirish mumkin. Tanasining shakli, rangida, xatti-harakatida himoyalangan hayvonlarga o'xshashlikni kuchaytiradigan kichik bir irsiy o'zgarishlari bo'lgan individlar yashab ketish va nasl qoldirish uchun foydali o'zgarishlari bo'lmagan individlarga karaganda ko'prok imkoniyatga ega bo'lgan. Foydali o'zgarishlar yashash uchun kurashda tabiiy tanlanish ta'sirida avloddan-avlodga kuchayib borib, takomillashgan va himoyalangan hayvonlarga o'xshashlik belgilarining to'planishiga olib kelgan. Har bir moslanish va ularning butun kompleksi bir kancha avlodlardagi yashash uchun kurash va tanlanish prosessida irsiy o'zgaruvchanlik asosida yuzaga keladi. Organizmlarning moslashganligi evolyusiyani harakatlantiruvchi kuchlarning mazkur yashash sharoitidagi ta'siri natijasidir. Moslanishlar paydo bo'lishini tushuntirish uchun Darvin bergan izoh shu masala xususidagi Lamark tushunchasidan tubdan farq qiladi. Hayvonlar tanasining rangi yoki shaklini o'zgartirishni «mashq qilgan» va shuning natijasida moslashib olgan, deb taxmin kilib bo'lmaydi. Organizmlarning bir-biriga o'zaro moslashganligini, masalan, ishchi asalarilar xartumining ular changlaydigan gul tuzilishiga mos bo'lishini ham bu bilan tushuntirib bo'lmaydi: ishchi asalarilar ko'paymaydi, ona asalarilar esa garchi nasl bersa ham xartumini «mashq qildira olmaydi», chunki ular guldan chang yig'maydi. Organizm ularda faqat foydali irsiy o'zgarishlar paydo bo'lishi yo'li bilan bevosita moslashib oladi, degan nazariya pirovard natijada azaldan maqsadga muvofiqlik to'g'risidagi tushunchalarga borib taqaladi. $\mathrm{Bu}$ nazariyaning idealistik xarakterini Darvinning evolyusiyani harakatlantiruvchi kuchlar to'g'risidagi ta'limoti asosida ochib berish mumkin.

Organizmlardagi moslanishlarning nisbiyligi. Organizmning tuzilishi va funksiyalarining maqsadga muvofiqligi uning faqat konkret tashqi muhit bilan munosabatlarida ifodalanadi. Xar qanday moslanish u evolyusiyani 
harakatlantiruvchi kuchlar ta'sirida qanday sharoitda yuzaga kelgan bo'lsa, xuddi shunday sharoitdagina organizmlar omon qolib, yashab ketishi uchun imkon beradi. Lekin bunday sharoitda ham u nisbiy bolladi. Oftob charaqlab turgan qish kunida oq kaklik qorga tushgan soyasi bilan o'zini sezdirib qo'yadi. O'rmonda qorda ko'zga chalinmaydigan oq quyon daraxtlar tanasining qora fonida ko'rinadigan bo'lib qoladi. Hayvonlardagi instinktlarni kuzatish bir qancha hollarda ular nisbiy ekanligini ko'rsatadi. Tungi kapalaklar, garchi nobud bo'lsa ham, o'zini olovga uradi. Ularni instinkt olovga jalb qiladi: ular asosan tunda yaxshi sezilib turadigan ochiq rangli gullardan nektar yig'adi. Organning tor doirada ixtisoslashganligi organizmning halokatiga sabab bo'lishi mumkin. Uzun qanot qaldirg'och tekis joydan ko'tarilib, uchib keta olmaydi, chunki uning qanotlari uzun va oyoklari juda kalta bo'ladi. U biror narsaning chetidan, xuddi tramplindan sakragandek sakrasagina, uchib keta oladi. O'simliklarning xayvonlarga yem bo'lishiga to'sqinlik qiladigan moslanishlari nisbiydir Tikanaklar bilan ximoyalangan o'simliklarni, odatda, mollar chetlab o'tadi, ammo yantoqni tuya, echkilar, och qolgan qoramollar xush ko'rib yeydi. Xar xil turlarga mansub organizmlarning, masalan, suvo't bilan zamburug'ning lishaynikda birga yashashi-simbiozning foydasi ham nisbiydir. Ba'zan lishaynikning zamburug' iplari o'zi bilan birga yashaydigan suvo'tlarni nobud qiladi. Organizmlarda foydasiz organ va belgilar uchraydi, masalan, otlarning grifel suyaklari, hech qachon suvga tushmaydigan tog' g'ozlarining barmoqlari orasidagi pardalar, maymun va odamda boladigan uchinchi qovoq qoldiqlari shular jumlasidandir. Mana shu va boshqa ko'pgina faktlar moslanishlar mutlaq bo'lmay, nisbiy ekanligini ko'rsatadi.

Yorug'lik. Sayyoramizdagi barcha organizmlar yorug'lik energiyasini Quyoshdan oladi. Erga etib kelayotgan yorug'lik oqimi ming angestremdan (1Ang10-8 sm) qisqa to'lqin uzunligidan tortib bir necha ming metrlar bilan o'lchanadi. Er yuzasiga yorug'likning 3900 dan 7700 angestremga qadar to'lqin uzunligidagi qismi yoki Quyosh radiatsiyasining $50 \%$ ga yaqini etib keladi. Faqatgina ultrabinafsha va infraqizil nurlar hamda spektrning ko'rinadigan etti xil nurlari hayvonlar hayotida muhim rol o'ynaydi. Hayvonlar yorug'lik energiyasini to'g'ridan-to'g'ri va bog'langan holda oziqlanishi orqali qabul qiladi (To'lqin uzunligi: 0.29-0.40 Nm li ultrabinafsha 
nurlar, $0.40-0.75 \mathrm{Nm}$ li ko'rinadigan oq nurlar va $0.75-1.0 \mathrm{Nm}$ li infraqizil nurlar).

Yorug'lik hayvonlarning ko'rishi, tevarak atrofni ilg'ashi, fazoda mo'ljal olishida asosiy rolni o'ynaydi. Hayvonlar ko'zlari orqali tashqi olam haqidagi axborotni qabul qiladi va unga tegishli javob reaktsiyasini qaytaradi. Hayvonlardagi ko'rish organlarining mukammallashuvi evolyutsiya jarayoni bilan hamohang ketgan. Ko'rish va uning qamrovi ayni guruh hayvonlarning evolyutsion rivojlanish darajasiga bevosita bog'liq bo'ladi. Umurtqasizlardagi eng sodda ko'zlar yorug'likni oddiygina ilg'ash organlari bo'lsa, sodda hayvonlarda bu tsitoplazmaning bir qismi sifatida shakllangan. Yorug'likni qabul qilish ko'rish pigmentlarining o'zgarishi, ya'ni fotokimyoviy reaktsiyalar hisobiga amalga oshadi. Ayrim sodda tuzilgan ko'zlar yorug'lik to'lqinlarining o'zgarishlaridangina (masalan, kun va tun almashinishi) dalolat beradi.

Mukammal ko'rish jarayoni murakkab darajada tuzilgan ko'zlargagina xos xususiyatdir.

Nisbatan sodda tuzilganligi bilan ajralib turuvchi o'rgimchak ko'zlari atrofidagi narsalarni 1-2 sm masofadangina payqay oladi. Eng yuqori darajada tuzilgan ko'zlar umurtqalilarda, boshoyoqli mollyuskalarda va hasharotlarda bo'ladi. Hasharotlardagi murakkab ko'z va uning ahamiyati orqali predmetlarning shaklini, rangini, joylanish uzoqligini farqlay oladi. Baliqlarda teleskopik ko'rish, amfibiyalarda harakatdagi narsani ko'rish, qushlarda $1 \mathrm{~km}$ dan ham, 1 sm dan ko'rish orqali narsa va buyumlarni farqlash mavjud.

Mukammal ko'rish insonlarga, primatlarga, ayrim qushlar - lochin, boyo'g'li, burgutlar va tasqaralarga xos. Odam va primatlarda:

- bir vaqtda 2 ko'z bilan qarash;

- predmet rangini, shaklini aniq ajratish;

- ungacha bo'lgan masofani aniq hisoblash xos.

Murakkab darajada tuzilgan ko'zlarning sezgirlik imkoniyati ham yuqori bo'ladi. Qorong'ulikka moslashgan inson ko'zlari 5 kvant jadallikdagi yorug'likni qabul qila oladi, bu esa ulardagi fizikaviy imkoniyat chegarasini ham belgilaydi. Hayvonlarning yorug'lik spektridagi ranglarni ajrata olish imkoniyati turlicha. Insonlar qizil, zarg'aldoq, yashil, ko'k-yashil, ko'k-binafsha ranglarni ajratadi. Ular ultrabinafsha va infraqizil nurlarni payqay olmaydi. Ayrim hayvonlar (kalmarlar, ilonlar) yorug'lik spektridagi infraqizil nurlarni 
osonlik bilan ajratadi va qorong'ulikda ham o'z o'ljasini bemalol topa oladi. Asalarilar ko'zga ko'rinmas ultrabinafsha nurlarni payqasa-da, qizil ranglarni ajrata olmaydi.

Ko'pchilik hasharotlarning tinim davridan chiqib faol hayot kechirishiga o'tishi yorug'lik kuchiga va haroratga bog'liq bo'ladi. May qo'ng'izi (Melolontha hippoicastani)ning g'umbakdan chiqishi quyoshning botishi soatiga to'g'ri keladi va bu muddat bahordan yozga tomon sekin asta kechikib boradi. Kunlik hasharotlardan Oligoneuriella rhenana havo harorati Q15오 bo'lib, kun botayotgan paytda uchib chiqadi. Uning rivojlanishida yorug'lik va harorat o'rtasidagi mutanosiblik muhim ahamiyatga ega. Yorug'lik kuchining o'zgarishi hayvonlarning fe'l-atvor reaktsiyalariga ham ta'sir qiladi. Masalan, kun tig'iga kelganda cho'l agamasi yulg'un, saksovul kabi butalarining yuqori yaruslariga chiqib olib jon saqlaydi. Harakatda bo'ladigan hayvonlar o'ziga joy tanlashda yoritilish darjasini ham hisobga oladi. Kecha-kunduz davomidagi faollik uchun ma'lum darajadagi yorug'lik talab etiladi. Hayvonlar kunduzgi, tungi, g'ira-shirada faol hayot kechiruvchilar ekologik guruhlariga ajraladi. Ularning faolligi mavsumga, iqlim sharoitlari va yorug'lik jadalligiga qarab o'zgarishi mumkin. Masalan, cho'l va chala cho'l mintaqalarida yashovchi yumronqoziqlar ertalab va kechki paytlarda aktiv faoliyatda bo'ladi, kunduzgi paytda uyalarida yashirinib yotadi. Sharoitga bunday moslanish ushbu mintaqalarda yashayotgan hasharotlar, sudralib yuruvchilar va sut emizuvchilarga ham xos.

Yorug'likning kunlik va mavsumiy o'zgarishlari hayvonlarning urchishiga, migratsiya va boshqa biologik maromlariga o'z ta'sirini o'tkazadi. Ba'zi hasharotlar kun qisqarishi natijasida ko'payish jarayonini to'xtatadi, kun uzayishi bilan esa ko'payish qayta tiklanadi.

Namlik. Suv - tiriklikning eng muhim va ajralmas tarkibiy qismi. Hayvonlar tsitoplazmasining 70-90\%, qo'ng'izlar va kapalaklar lichinkalari og'irligining $50 \%$ va ayrim meduzalar tanasining $90 \%$ ini suv tashkil etadi. Biokimyoviy jarayonlarning borishida suv muhit vazifasini bajaradi. Shuning uchun ham namlikning me'yorida saqlanishi barcha organizmlar uchun birinchi darajali ahamiyat kasb etadi.

Sovuq harorat, qor qoplami, namlik bilan birga yorug'likning jadalligi, ayniqsa ultrabinafsha nurlar hayvonlar rangiga jiddiy ta'sir etadi. To'q rang 
hayvonlarni ultrabinafsha nurlardan himoya qiladi, boshqa nurlarni o'ziga yig'ib tana haroratini me'yorida saqlanishiga yordam beradi.

Namlikka bo'lgan ehtiyoji va yashash joylari bo'ylab taqsimlanishiga muvofiq hayvonlar quyidagi ekologik guruhlarga ajraladi:

Gidrofillar - hayoti doimo suvda o'tuvchi bu guruhga gidrobiontlar kiradi.

Gigrofillar - yashash joylaridagi, havodagi nisbiy namlik yuqori yoki shu darajaga yaqin bo'lgan sharoitda uchraydigan hayvonlar guruhi. Bu guruhga qator amfibiyalar, ko'pchilik qorin oyoqli mollyuskalar, yomg'ir chuvalchanglari, tuproq faunasining asosiy vakillari, g'orlarda yashovchi hayvonlar va baliqlar mansub.

Mezofillar - o'rtacha namlik sharoitida yashovchi hayvonlar. Ular mo"tadil va o'rta tog' mintaqalarida, madaniy landshaftlarda yashaydi. Mezofillar namlikni mavsumiy o'zgarishlariga birmuncha bardoshli bo'ladi.

Kserofillar - qurg'oqchil sharoitda yashashga moslashgan hayvonlar. Cho'l, chala cho'l va tog'oldi hududlarda uchraydigan ko'pchilik hasharotlar va sut emizuvchilar mazkur ekologik guruh vakillaridir. Kserofillarning barchasi quruq va qurg'oqchil iqlim sharoitlariga keng ma'noda adaptatsiyalangan. Masalan, Helix desertorum shilliqurti noqulay sharoitda yozgi tinim davriga o'tadi va uning "yozgi uyqusi" 4 yilga qadar davom etishi mumkin. Toshbaqalarda yozgi va qishki uyqu mavjud bo'lib, 6-7 oy uxlaydi.

Hayvonlar suvga bo'lgan talabini 4 xil yo'l bilan qondiradi.

1. Bevosita suvni ichish orqali. Ayrim reptiliyalar, sutemizuvchilar va qushlar suvni ko'plab istemol qiladi. Ayrim qushlar, tuyoqlilar, yirtqichlar, ko'rshapalaklar uzoq muddatli suvsizlikka chiday olmaydi va suv topish maqsadida uzoq masofalar bo'ylab uchib harakatda bo'ladilar. Qaldirg'och va jarqaldirg'ochlar suv havzasi bo'ylab uchib o'tadi va pastlab suvni qabul qiladi. Tuyalar 2-3 haftagacha suvsiz yura oladi, lekin bir suv ichganda 200 l gacha suv ichishi mumkin.

2. Ovqat orqali. Cho'l mintaqalarida yashovchi ko'pchilik hayvonlar (kemiruvchilar, antilopalar va boshqalar) hech qachon suv ichmaydi, ular uchun iste'mol qilinayotgan ozuqa suv manbai hisoblanadi (metabolitik suv deyiladi). Gul sharbati, qon bilan oziqlanuvchi hasharotlar ovqat bilan hatto ehtiyojidan ortiq suvni oladi. 
3. Teri orqali suvni qabul qilish. Amfibiyalar, ayrim hasharotlar va kanalar namlikni butun tanasi bo'ylab qabul qiladi. Kolorado qo'ng'izi, taxtakana havodagi nisbiy namlik minimal darajada bo'lgan sharoitda ham suvga bo'lgan ehtiyojini tanasi bo'ylab suvning adsorbtsiyalanishi ularning hayotida muhim ekologik ahamiyatga ega.

4. Metabolitik suv hisobiga yashash. Ya'ni organizmlardagi yog'lar, oqsillar, karbon suvlarning parchalanishidan ajralgan suvning assimilyatsiyalanishi. Qurg'oqchil iqlim sharoitida yashaydigan kemiruvchilar turli hasharotlar va tuyalar suvni shu usul bilan o'zlashtiradi. Qulay sharoitda cho'ldagi sut emizuvchilar va qushlarning tanasida yog' ko'plab to'planadi, noqulay mavsum boshlanganda esa, u sarflanadi. Yog'lar hayvonlar uchun nafaqat energiya manbai, balki suvga bo'lgan ehtiyojini qondiruvchi omil vazifasini ham bajaradi, chunki yog' oksidlanib parchalanganda ko'p suv ajraladi. Don, un, yog'och bilan oziqlanuvchi hasharotlar hayoti ham metabolitik suvga bog'liq.

Namlik teri orqali, nafas olish jarayonida, siydik, ter va ekskrementlar bilan transpiratsiyalanishi va bug'lanish mumkin. Namlikni yo'qotishga nisbatan hayvonlardagi moslanishlar xilma-xil bo'lsada, ularni 3 guruhga ajratish mumkin.

1. Suv sarfining kamayishi hayvonlardagi morfologik va fiziologik moslanishlar bilan bog'liq. Qurg'oqchil sharoitda yashaydigan hayvonlarning tanasi namlikni saqlashga yordam beradigan chig'anoqlar, tangachalar bilan qoplangan yoki himoya kosachasi bo'ladi, mollyuskalar esa chig'anoqlar bilan himoyalangan, cho'l hasharotlari tanasi qalin xitindan iborat. Lekin cho'lda yashovchi hayvonlarning barchasida ham shu singari morfologik moslanish kuzatilmaydi. Masalan, tekis oyoqli qisqichbaqasimonlarning vakili sanalgan "eshakqurt" quruqlikda yashaydi, tanasi yupqa-nozik xitin bilan himoyalangan. Ular Qoraqum va Qizilqum cho'llaridan tortib adir zonalarni ham egallagan.

Evolyutsiya jarayonida ichki nafas olish organlarining shakllanishi hayvonlarga suv sarfini birmuncha kamayishiga sabab bo'lgan. Quruqlik hayvonlarida jabralarni yo'qligi va hasharotlarda, ko'poyoqlilar va o'rgimchaksimonlar nafas olish organlari sifatida traxeyalar tizimini paydo bo'lganligi, mollyuskalarda "o'pkalar", amfibiyalarda o'pka va teri orqali nafas 
olish, reptiliyalar, qushlar va sut emizuvchilarda haqiqiy o'pkalarning taraqqiy etganligi unga misol bo'ladi.

Siydik va ter hisobiga suv sarfini kamaytirishda organizmlardagi azot almashinuvini muvofiqlashtirish muhim ahamiyatga ega. Oqsillar parchalanishi bilan ajralib chiqqan ammiakning juda kam miqdori ham organizm uchun zaharli ta'sirini ko'rsatadi. Uni zararlantirish uchun esa yuqori hajmdagi suv sarfi talab etiladi. Hayvonlardagi o'ziga xos moslanishlar ayni holatni oldini olishga xizmat qiladi. Jumladan, yuqori kontsentratsiyadagi siydikni ajratish ko'pchilik hayvonlardagi namlikni me'yorida ushlab turishiga nisbatan adaptatsiyalanish usullaridan biri. Masalan, reptiliyalar, hasharotlar va quruqlik mollyuskalarining ayrimlari quruq uratlarni ajratadi. Namlik etarli bo'lgan sharoitda yashaydigan hayvonlar siydigi tarkibidagi ammiakning umumiy ulushi sezilarli bo'ladi. Hasharotlar (shiralar) ozuqasi tarkibidagi suvning mo'lligi ular ajratayotgan suyuqlikda ammiak miqdorining yuqoriligi sabab bo'lgan. Sut emizuvchilar siydigida nisbatan zararsiz mochevina bo'ladi. U dissimilyatsiya mahsuloti sifatida qon zardobida va tana bo'shlig'ida yig'ilib, nisbatan yuqori kontsentratsiyada ajralib chiqadi. Siydik bilan birga zararli tuzlar ham chiqarib tashlanadi. Siydik va qon zardobi kontsentratsiyalari o'rtasidagi mutanosiblik suvni tejalish darajasini ham ifodalaydi. Masalan, siydikning plazmaga nisbatan kontsentratsiyasi odamlarda 4,2 marta ortiq, qo'ylarda bu ko'rsatkich 7,6 ga, tuyalarda - 8 ga va qo'shoyoqlarda esa 14 ga teng.

Cho'lda yashovchi tangachali sudralib yuruvchilar, toshbaqalar va qushlar, hasharotlar suvda yomon eriydigan siydik kislotasini ajratadi, o'rgimchaklar esa, guaninni chiqarib tashlaydi. Guanin va siydik kislotalari bilan eng minimal darajadagi suv ajraladi. Kemiruvchilar va antilopalarning tanasidagi kam miqdordagi ter bezlari, quruq najas ulardagi suv sarfi bir muncha past darajada ekanligidan dalolat beradi.

Namlikni yo'qotish chegarasi barcha organizmlarda bir xil darajada emas. Masalan, odamlar tanasidagi vaznga nisbatan $22 \%$ gacha suvni yo'qotish mumkin, lekin undan ortiqcha yo'qotganda odam halok bo'lishi mumkin. Bu chegara tuyalarda $27 \%$ ni tashkil etsa, qo'ylarda $23 \%$ va itlarda $17 \%$ ga to'g'ri keladi.

Suvning tejalishida organizmlardagi qator fiziologik moslanishlar ham 
muhim rol o'ynaydi. Jumladan, ayrim hayvonlarning ovqat qoldiqlari va najasi bilan chiqayotgan suv ichaklarda yana so'riladi, tashqariga bir muncha quruq holdagi najas chiqarib tashlanadi. Shuning uchun ham hayvonlarda namlikni iqtisod qilinish darajasi chiqindi tarkibidagi namlik me'yori bilan ham o'lchanadi. Masalan, yaylovdagi qoramol tezagining $100 \mathrm{~g}$ og'irligiga $566 \mathrm{~g}$ suv to'g'ri keladi, tuyalarda bu ko'rsatkich $109 \mathrm{~g} \mathrm{ni}$, suvsiz sharoitda esa $76 \mathrm{~g} \mathrm{ni}$ tashkil qiladi.

Nisbiy namlik past sharoitda yashovchi hasharotlarning ayirish tizimlari - malpigi naylarining oxirgi yo'g'on ichakning orqa devoriga tutashgan bo'lib, undagi suv so'rilishi orqali organizm ehtiyoji uchun qayta sarflanadi, ya'ni reabsorbtsiyalanadi. Namlik etarli bo'lgan sharoitda hayot kechiruvchi va suyuq ozuqa bilan oziqlanuvchi hasharotlarda esa reabsorbtsiya hodisasi kuzatilmaydi. Ular siydik orqali tashqariga har xil tuzlar va mochevina chiqaradi. Bu mazkur hasharotlarning ayirish tizimi bilan bog'lanmagan.

2. Metabolitik suvni o'zlashtirish quruq yoki namligi kam bo'lgan ozuqa bilan oziqlanib hayot kechiruvchi hayvonlarda kuzatiladi. Kunduz kunlari uyalarida jon saqlaydigan mayda kemiruvchilar faqat yuqori haroratdagina himoyalanmay, namlikni ortiqcha sarfini ham oldini oladi. Bu muddatda namlikka bo'lgan ehtiyoj metabolitik suv hisobidan qoplanadi. Afrika cho'llarida yashovchi chumolilarning lichinkalari tanasidagi yog'lar oksidlanishi natijasida ajralgan suv hisobiga yashaydi. Bundan tashqari ularning gemolimfalari tarkibidagi oqsillar yuqori osmotik bosimni ta'minlaydi, bu esa namlikning bug'lanishini sekinlashtiradi.

3. Ekologik va etologik adaptatsiyalar xilma-xil bo'lib, keng tarqalgan moslanishlardan biri cho'l hayvonlarining tungi tarzi yoki qulay yashash joylarini topish maqsadida bir joydan ikkinchi joyga ko'chishi va boshqalar misol bo'ladi.

Namlikning hayvonlarga ta'siri. Namlik hayvonlarning umriga, rivojlanishiga serpushtligiga, fe'l-atvoriga: geografik tarqalishi va yashash joylarini tanlashiga, shuningdek populyatsiya miqdor zichligiga ta'sir ko'rsatadi.

Hayvonlar umrining uzunligi va rivojlanishi namlikka bog'liq bo'ladi. Jumladan, cho'l hayvonlari uzoq muddatli suvsizlikka chiday oladi, lekin baqalar nisbiy namlik past sharoitda tezda nobud bo'lib ketadi. Namlik etarli 
bo'lganda uy pashshasining rivojlanishi jadal ketsada, lekin namlik kamayishi bilan bu jarayon sekinlashib boradi va butunlay to'xtashi mumkin.

Namlik va hasharotlar serpushtligi o'rtasida ma'lum bog'liqlik mavjud. Masalan, Panolis flammea kapalagining 80\% urg'ochilari yuqori namlik sharoitida qo'shila olmaydi, namlikning ko'tarilishi kapalak serpushtligini susaytiradi.

Namlikning o'zgarishi hayvonlarning fe'l-atvorida ham o'z ifodasini topadi. Jumladan, qon so'ruvchi chivinlar havoning nisbiy namligi $40 \%$ dan past sharoitda issiqqonli hayvonlarga "hujum" qilmaydi va qon so'rishdan to'xtaydi. Chiriyotgan to'nkadagi namlik ko'tarilgan taqdirda, unda oziqlanayotgan ksillofag qo'ng'izlar jadallik bilan to'nkani tark etadi va boshqa qulay yashash joyiga "qochadi".

Hayvonlarning geografik tarqalishi va yashash joylarini tanlashi boshqa omillar bilan birga namlikka ham bevosita bog'liq bo'ladi. Jumladan, hayvonlarning vertikal mintaqaviy taqsimlanishi va yashash joylarini tanlashda ushbu holat asosiy ahamiyatga ega.

Namlik va harorat birgalikda hayvonlarning populyatsiya miqdor zichligiga sezilarli ta'sir etadi. Bahor va dastlabki yoz oylarida nisbiy namlik yuqori bo'ladi, bu esa hasharotlarni ko'plab ko'payishiga imkoniyat yaratadi va ularning miqdor zichligi yuqori darajaga ko'tariladi. Yozning ikkinchi yarmida quruq va issiq havo hasharotlar populyatsiya miqdor zichligini keskin pasayishiga olib keladi.

Harorat. Atrof-muhit harorati hayvonlarning ko'payishiga, rivojlanishiga, yashovchanligiga, tana haroratiga va moddalar almashinuvi jarayoniga, shuningdek populyatsiya miqdor zichligi hamda turli yashash joylari bo'ylab taqsimlanishiga bevosita yoki bilvosita ta'sir ko'rsatadi.

Haroratni hayvonlar butun tanasi bo'ylab qabul qilib, ortiqcha issiqlikni atrof-muhitga chiqarib tashlaydi. Bu holat muhit haroratiga bog'liq holda namoyon bo'ladi va organizmning issiqlik darajasini ifodalaydi.

Ma'lumki, Vant-Goff qoidasiga muvofiq, haroratning $10^{\circ} \mathrm{S}$ ga ko'tarilishi kimyoviy jarayonlarni 2-3 martaga tezlashtiradi. Lekin, biologik jarayonlar uchun bu qoida haroratning muayyan chegarasidagina amal qiladi, harorat ko'rsatkichining ushbu chegarasidan chetga chiqishi organizmlarda borayotgan moddalar almashinuvini keskin susaytiradi. Masalan, reptiliyalar 
tuxumining rivojlanishi bilan harorat o'rtasida to'g'ri chiziqli bog'lanish yo'q. Huddi shuningdek, haroratni ko'tarish bilan tovuq tuxumining rivojlanishini tezlashtirish mumkin emas, aksincha, jo'jani rivojlanib voyaga etishi uchun 21 kun davomida doimiy 40-410S harorat zarur.

Hayvonlardagi har bir hayotiy jarayonning borishi uchun haroratning quyi va yuqori pog'onasi, optimum chegarasi zarur. Masalan, gulmoy balig'ining uvildirig'i $0^{\circ} \mathrm{S}$ da, chavaqlar esa Q ${ }^{20} \mathrm{~S}$ da rivojlana boshlaydi. Agar harorat shunday tursa, chavaqlarining chiqishi uchun 205 kun, Q505 da 85 kun va Q10요 da esa 41 kun zarur bo'ladi. Kolorado qo'ng'izi Q $12^{\circ} \mathrm{S}$ da rivojlana boshlaydi, Q 250S haroratda uning lichinkalik davri 14-15 kun davom etadi, Q30으 li sharoitda lichinka bor yo'g'i 5,5 kunda voyaga etadi. Q33오 da u rivojlanishi, fiziologik va biokimyoviy jarayonlari harorat o'zgarishiga monand to'g'ri chiziqli ko'tarilmaydi, aksincha, ularning har biri turli yo'nalishdagi giperbola chizig'ini hosil qiladi. Ikkinchi tomondan, bu jarayonlarning borishi birgina haroratga bog'liq bo'lib qolmay, ularga boshqa ekologik omillar ham ta'sir etadi.

Barcha omillar singari haroratning to'g'ridan-to'g'ri ta'siri jamoadagi boshqa hayvonlar hayotida, yashash joylarini tanlashida va boshqa holatlarda o'z ifodasini topadi.

Harorat hayvonlar uchun signal vazifasini o'taydi. Uning o'zgarishi bilan hayvonlar yashashi uchun qulay joy izlaydi, bir mintaqadan ikkinchisiga ko'chadi, ularning hayotida va geografik taqsimlanishida qator o'zgarishlar ro'y beradi.

Hayvonlardagi issiqlik almashinuvi nerv-gumoral yo'l bilan boshqariladi. Issiqlik almashinuvining poykilotermlik va gomoyotermlik usullari farqlanadi.

Poykiloterm hayvonlarda o'zgaruvchan tana harorati va moddalar almashinuvi kuzatiladi. Muhit haroratiga monand poykilotermlarning tana harorati ham o'zgarib turadi. Gomoyotermlardagi moddalar almashinuvi bir me'yorda ketadi va ular doimiy tana haroratiga ega bo'ladi.

Poykiloterm va gomoyotermlardan tashqari oraliq guruh vakillari geterotermlar ham kuzatiladi. Geterotermlar mavsumiy faol hayot kechirganda, ular gomoyotermlar singari tana haroratini doimiy saqlaydi, lekin qishki (yumronqoziqlar, olmaxonlar, qo'shoyoqlar, ayrim qushlar) yoki 
chuqur uyquda (ko'rshapalaklar, bo'rsiqlar, ayiqlar, kolibri qushlari, jarqaldirg'och va boshqalar) ulardagi modda almashinuvi susayadi, tana harorati muhit haroratidan biroz farq qiladi, xolos.

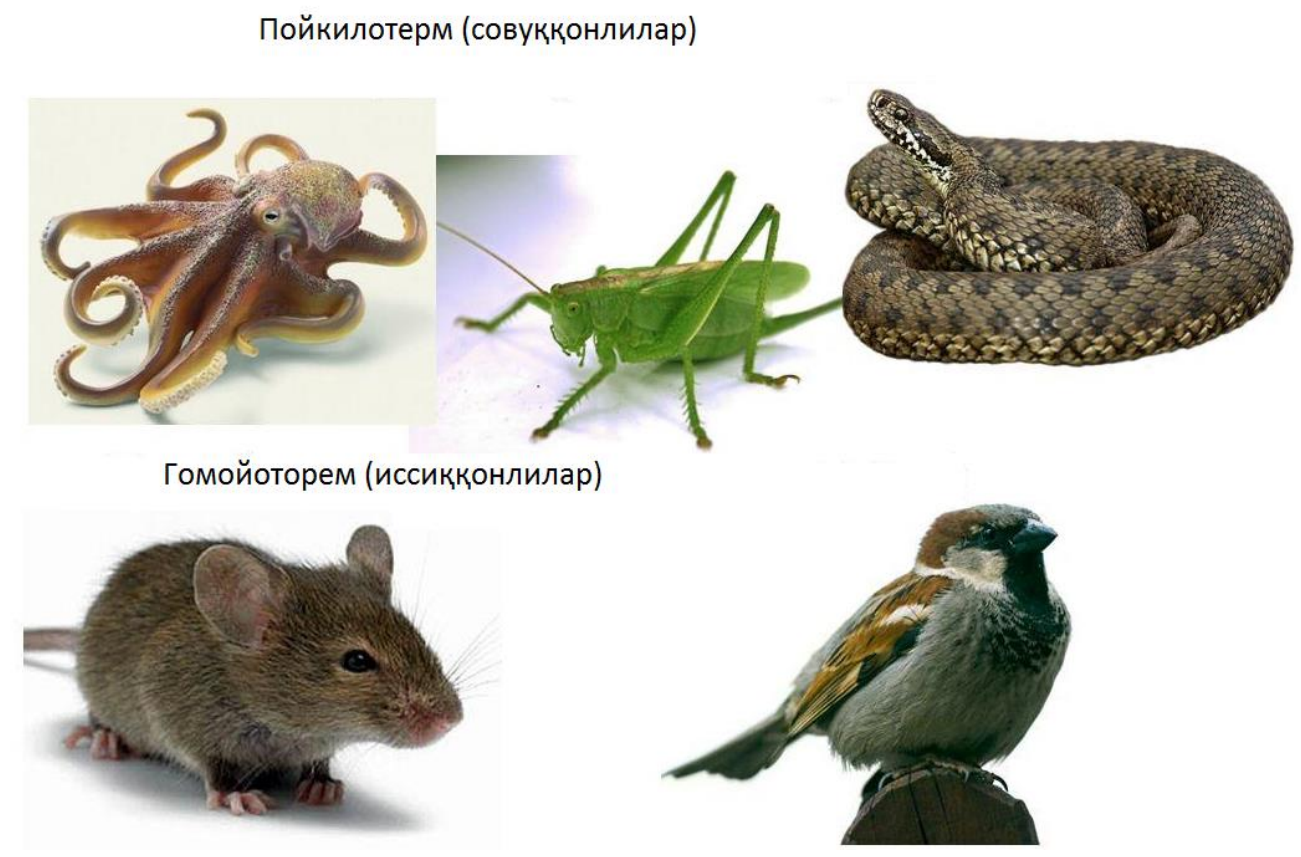

3-rasm. Issiqqonli va sovuqqonli hayvonlar

O'simliklardan farqliroq hayvonlar muskullar bilan ta'minlangan, shu sababdan ular o'zining ichki haroratiga nisbatan ham yuqoriroq darajadagi issiqlikni ishlab chiqara olish imkoniyatiga ega. Muskullar qisqarishida hosil bo'lgan energiya boshqa organ yoki to'qimalar harakati davomida ajaralayotgan issiqlikdan bir necha barobar yuqori bo'ladi, muskullar qanchalik tez harakat qilar ekan, ajaralayotgan issiqlikning miqdori ham shuncha yuqori bo'ladi. Hayvonlar tana haroratini vaqtincha yoki doimiy bir me'yorda boshqara olishi mumkin.

Hayvonlardagi haroratga adaptatsiyalanishning asosiy yo'llari kimyoviy va fizikaviy termoregulyatsiyadan iborat, misol tariqasida kunduzgi va tungi aktiv hayvonlarni olish mumkin.

Hayvonlarning fe'l-atvor harakatlari asosida tana haroratini idora etilishi. Tashqi muhit haroratining pasayishiga javoban faol ravishda tanadan issiqlik ajralishi yoki issiqlik ajratishni atrof-muhit haroratining o'zgarishiga qarab muvofiqlashishi kimyoviy termoregulyatsiya deyiladi.

Fizikaviy termoregulyatsiya tanadan issiqlik ajratishning o'zgarishi, ya'ni tana issiqligining yig'ilishi yoki aksincha ortiqcha bo'lsa, chiqarib yuborishdir. 
Fizikaviy termoregulyatsiya organizmlarning morfologik va anatomik tuzilishlari bilan ifodalanadi. Hayvonlarning yung qoplamlari, qon-tomir tizimining joylashish xususiyati, yog' qatlamlari va zahiralari, teri yoki nafas yo'li orqali suv bug'latishni boshqarish fizikaviy termoregulyatsiyani belgilovchi omillardan hisoblanadi.

Hayvonlar bir mintaqadan boshqasiga siljishi, yashash joylarini o'zgartirishi va boshqa murakkab fe'l-atvor harakatlari asosida noqulay sharoitlarga moslashadi. Ko'pchilik hayvonlar uchun fe'l-atvor harakatlari asosida haroratni idora etilishi noqulay sharoitlarga moslanishning yagona va samarali usulidir.

Poykiloterm hayvonlar hozirda harorat kam o'zgaradigan suv havzalaridagina emas, balki harorat tez-tez o'zgaruvchan mintaqalar quruqlik, mo"tadil iqlim sharoitida va hatto Arktikada ham uchraydi.

$\mathrm{Bu}$ holat poykilotermlarni noqulay iqlim sharoitlariga ham moslashganligidan dalolat beradi. Poykilotermlarning hayoti atrof-muhit haroratiga mos ravishda o'zgarib turadi. Masalan, Bodenxeylir Shistocerea gregaria chigirtkasining faolligi va haroratning o'zgarishi o'rtasidagi bog'liqlikni tadqiq etib, quyidagi bosqichlarni farqlagan:

- past haroratli sharoitdagi karaxtlikdan chiqish;

- mo'ylovlar va panjalarning sust harakati;

- ishonchsiz o'rmalash;

- me'yoridagi harakatlar;

- o'ta faollik;

- kuchli qo'zg'alish;

- yuqori harorat ta'siridagi karaxtlik;

- issiqdan nobud bo'lish.

ushbu bosqichlarning harorat ko'rsatkichlari 1-jadvalda ifodalangan.

2-jadval

Schistocerea gregaria chigirtkasi faolligining turli bosqichlari mos harorat ko'rsatkichlari (Bodenheimer, 1938)

\begin{tabular}{|l|l|l|c|c|c|c|c|c|}
\hline \multirow{2}{*}{ Tur nomi } & \multicolumn{7}{|c|}{ Faollik bosqichlari } \\
\cline { 2 - 9 } & 1 & 2 & 3 & 4 & 5 & 6 & 7 & 8 \\
\hline Etuk erkagi (mart oyida) & 4,9 & 10,5 & 18,0 & 20,1 & 32,6 & 41,8 & 48,4 & 50,5 \\
5 yoshli lichinkasi & 5,5 & 7,6 & 20,7 & 26,0 & 36,9 & 45,3 & 49,2 & 51,0 \\
\hline
\end{tabular}


Yuqori haroratga bardoshlilik hayvonlardagi ekologik va evolyutsion moslanishlardan biri. Masalan, dengiz umurtqasizlari chuchuk suv hayvonlariga nisbatan yuqori haroratga chidamsiz bo'ladi. Jumladan, ko'k baqa Q41-440S ga bardosh bera oladi, lekin dengiz umumrtqasizlarining ko'pchiligi Q30-3200 li sharoitga chidaydi xolos, birgina aktiniyalar Q3805 ga qadar bo'lgan haroratni osonlik bilan enga oladi.

Quruqlikda yashovchi ayrim hasharotlar esa Q45-50²S li sharoitda ham ma'lum muddat tirikligini saqlaganligi aniqlangan. Poykilotermlar sovuqqa chidamliligi ularning yashash muhiti va tarzi, rivojlanish bosqichlariga bog'liq bo'ladi. Sodda hayvonlar $-15^{\circ} \mathrm{S}$ da ham tirikligini saqlaydi. Jumladan, infuzoriya tufelkaning kipriklari $-12^{\circ} \mathrm{S}$ da ham harakatda bo'ladi. Kapalkalar -8 dan $-20^{\circ} \mathrm{S}$ ga qadar bo'lgan sovuqqa bardosh bera oladi. Osiyo chigirtkasining tuxumlari $-30^{\circ} \mathrm{S}$ da ham nobud bo'lmaganligi aniqlangan. Hayvonlarning sovuqqa chidamlilik darajasi tuzlar, glikogen, yog'lar va bog'langan suv miqdoriga bog'liq. Masalan, tunlam kapalaklari tanasidagi yog'lar ulushi 18,2$19,4 \%$ bo'lganda, ular $-5.75^{\circ} \mathrm{S}$ li sovuqni hech qanday qiyinchiliksiz o'tkaza oladi.

Poykilotermlar atrof-muhit haroratiga moslanish xususiyatlariga ko'ra 2 guruhga ajraladi: stenotermlar va evritermlar.

Stenotermlar nisbatan doimiy haroratli sharoitda yashashga ixtisoslashgan. Ular haroratni sezilarli o'zgarishlariga chidamsiz hayvonlar. Stenotermlar o'rtasida kriofillar va termofillar farqlanadi. Masalan, ayrim termofil qisqichbaqasimonlar Q45-48 $\mathrm{S}$ li issiq buloqlarda yashaydi, agar suv harorati $\mathrm{Q} 30^{\circ} \mathrm{S}$ ga qadar pasaysa, ular butunlay nobud bo'ladi. Okeanlar tubida yashaydigan ko'pgina hayvonlar kriofillar bo'lib, $0^{\circ} \mathrm{S}$ da hayot kechiradi. Ximolay tog'larining muzliklarida uchraydigan dumoyoqlilar - $10^{\circ} \mathrm{S}$ sovuqda yashashga moslashgan, shu sababdan ularni inson qo'lining harorati ham halok qilishi mumkin.

Evriterm harorat turli darajada bo'lgan sharoitda ham bemalol hayot kechira oladi. Jumladan, qorinoyoqli mollyuskalarning ayrimlari - $1^{0} \mathrm{~S}$ dan $\mathrm{Q}$ $60^{\circ} \mathrm{S}$ ga qadar o'zgaruvchan sharoitda uchrashligi ma'lum. Yo'lbars Hindistonning tropik o'rmonlaridan Ussuriya o'lkasiga qadar tarqalgan, shuningdek, u Ximolayning dengiz yuzasidan $4000 \mathrm{~m}$ balandlikdagi tog' 
cho'qqilarida ham uchraydi.

Gomoyotermlarga nisbatan poykilotermlarning hayotiy jarayonlari bir muncha sust ketadi. Bir xil tana haroratiga ega bo'lgan gomoyoterm va poykiloterm hayvonlardagi moddalar almashinuvi jadalligi qiyoslanganda, bu jarayon poykilotermlarda bir necha barobar past darajada borishligi aniqlangan. Masalan, cho'l iguanasi Q370S haroratda kattaligi o'ziga teng bo'lgan kemiruvchilarga nisbatan kislorodni 7 marta kam sarflagan. Bu holat poykilotermlardagi issiqlik ajratilishi ancha sust ekanligidan dalolat beradi. Shuningdek, ulardagi kimyoviy termoregulyatsiya bir muncha takomillashmaganligini ifodalaydi.

Poykilotermlarda fizikaviy termoregulyatsiya ham yaxshi shakllanmagan. Muhit harorati pasayishi bilan poykilotermlarning hayotiy jarayonlari keskin pasayadi va ular karaxt holatga o'tadi. Biokimyoviy adaptatsiyalanishning hosilasi bo'lgan karaxtlik noqulay sharoitga moslanishi o'ziga xos bo'lib, bunday holatda poykilotermlar ancha past haroratga ham bemalol bardosh bera oladi. Poykilotermlarning karaxtlikdan chiqishlari va faol holatga o'tishlari uchun ular tashqaridan issiqlik olishlari shart.

Poykilotermlar fe'l-atvor harakatlari bilan tanada yig'ilayotgan issiqlikni ko'paytirishlari yoki kamaytirishlari mumkin. $\mathrm{Bu}$ guruhga mansub organizmlar yashash joylari va fazodagi holatini o'zgartirish asosida iqlim o'zgarishiga o'z munosabatini bildiradilar. Masalan, chigirtkalar ertalab quyoshga yon tomonini toblasa, tushda elkasini to'g'rilaydi. Cho'llarda quyosh yuqori nuqtaga kelganda, barcha hayvonlar uyalarida yoki salqin joylarga yashirinib, tunda esa faol bo'ladi. Qish mavsumini ko'pchilik hayvonlar yaxshi himoyalangan uyalarida o'tkazadi yoki qishki uyquga ketadi. Jamoa bo'lib yashovchi hasharotlar - arilar, chumolilar, termitlar uyalarini shunday quradilar-ki, undagi harorat va namlik bir me'yorida saqlanib turadi.

Poykiloterm hayvonlarning ayrimlarida kimyoviy termoregulyatsiya bir oz bo'lsada, taraqqiy etgan, ular muskullarni jadal qisqarishi hisobiga tana haroratini ko'tarishi mumkin. Masalan, pahmoq arilarning tez qanot qoqishi tufayli tana harorati Q32-33오 ga qadar ko'tariladi va shu tufayli ular sovuq havoda ham bemalol oziqlanib harakatda bo'ladi.

Ba'zi poykilotermlarda fizikaviy termoregulyatsiyaning elementlari ifodalangan, ya'ni ular tanasi orqali suvni bug'lantirib haroratni boshqara 
oladi. Masalan, ko'k baqa quruqlikda, Q20요 da soatiga $7770 \mathrm{~J}$ issiqlik yo'qotishi mumkin, bu esa uning ishlab chiqayotgan issiqligidan 300 marta katta ko'rsatkichdir. Harorat ko'tarilib, yuqori kritik nuqtaga kelganda, sudralib yuruvchilar qiyin nafas ola boshlaydi va og'zini ochib suvni bug'latadi.

Evolyutsiya jarayonida gomoyotermlik poykilotermlikdan rivojlangan. Gomoyotermlik yirtqichlar, primatlar va tuyoqlilarda yuksak darajada taraqqiy etgan bo'lsada, lekin bu xususiyat mavsum va kun davomida ma'lum chegarada o'zgarib turadi. Gomoyotermlik kloakali va xaltali sut emizuvchilarda, yuqori sut emizuvchilarning bolalarida va qushlarning jo'jalarida bur muncha sust ifodalangan. Gomoyotermli qushlar va sut emizuvchilarning muhit bilan bo'ladigan eng murakkab munosabatlarining hosilasi hisoblanadi. Gomoyotermlik ularga barcha sharoitlarda ham yashay olish imkonini beradi va ular tashqi muhit omillari o'zgarishlariga qaram bo'lmaydi, mavsumning turli davrlariga oson moslasha oladi. Bu xususiyatlar gomoyotermlilardagi mukammal organlar tizimi, jadal harakat organlari, qon aylanish va nerv tizimlari, yog' qatlamlari, yung, par qoplamlari va boshqalar hisobiga shakllangan.

Gomoyotermlardagi termoregulyatsiya mexanizmlari har bir turning o'zida xilma-xil bo'ladi. Bu esa tana haroratini kafolatli bir darajada ushlab turish imkoniyatini beradi. Shu sababdan shimoliy mintaqalarda tarqalgan oq tulkilar, kakliklar va quyonlar tana harorati va atrof-muhit harorati o'rtasidagi farq $70^{\circ} \mathrm{S}$ dan yuqori bo'lgan sharoitda ham bemalol yashay oladi. Gomoyoterm hayvonlar yuqori haroratga ham oson moslashadi. Ingliz olimi Ch.Blegden va uning shogirdlari, tajribadagi it bilan Q126오 daqiqa bo'lganlarida, bu holat ularning sog'liqlariga salbiy ta'sir ko'rsatmagan.

Gomoyoterm hayvonlarda kimyoviy va fizikaviy termoregulyatsiya mukammal shakllangan. Evolyutsiya jarayonida kimyoviy termoregulyatsiya mohiyatan bir muncha erta yuzaga kelgan, lekin tarixiy taraqqiyot davomida uning ahamiyati fizikaviy termoregulyatsiyaga nisbatan bir oz bo'lsada kamaygan. Sut emizuvchilarning filogenetik qatori bo'ylab yuqorilashgan sari fizikaviy termoregulyatsiya mexanizmlari xilma-xilligi ortgan va takomillashib borgan. Shimol hayvonlari tanasini qoplab turgan zich parlar, patlar va yunglar haroratning tana haroratiga yaqin darajada bo'lishiga yordam beradi. 
Shu sababdan shimol tulkilari qishda yozdagiga nisbatan ham oz ovqat iste'mol qiladi. Suvda yashovchi sut emizuvchilarning tanasi 7-9 sm qalinlikdagi yog' qatlami bilan o'ralgan va tana og'irligining 40-50\% yog'ga to'g'ri keladi. Shuning uchun ham tyulenlar qorda soatlab yotgan taqdirda ham sovuqni sezmaydi.

Haroratning boshqarilishida tana katta-kichikligi ham ma'lum ahamiyatga ega. Ekologiya va zoogeografiyada keng qo'llaniladigan Bergman qoidasiga muvofiq bir turning shimoliy formalari ancha yirik bo'lsa, janubga tomon tanasi kichiklashib boradi. Jumladan, issiq o'lkalarda yashovchi ko'pgina sut emizuvchi hayvonlar vazni, ichki organlari sovuq o'lkalarda yashovchi shu xildagi hayvonlarnikiga nisbatan engil va kichikdir.

D.Allen qoidasiga muvofiq esa, ko'pchilik sut emizuvchilar va qushlarning dumlari, quloqlari va tumshuqlari Janubga tomon kattalashib boradi. Masalan, shimol tulkisining quloqlari va dumlari, Afrikadagi fenek tulkilarnikiga nisbatan bir muncha kichik va kalta bo'ladi, ya'ni turli iqlim zonasida yashaganliklari uchun ular tashqi ko'rinishlari, ayniqsa, quloqlarning shakli, dumlari katta-kichikligi bilan bir-birlaridan keskin farqlanadi. Afrika fillarining quloqlari Hindiston fillarinikiga nisbatan bir muncha yirik bo'ladi. Shuni ta'kidlash lozimki, janubiy mintaqada yashaydigan ko'pgina hayvonlarning quloqlari maxsus termoregulyatsiya organi vazifasini bajaradi. Muhit haroratiga moslanishda hayvonlarning fe'l-atvor harakatlari muhim o'rin tutadi. Hayvonlarning boshpana topishi, uyalar, inlar qurishi, mintaqalar bo'ylab ko'chib yurishi va boshqa harakatlar fe'l-atvorlar orqali boshqariladi.

Tana haroratini idora etilishida gala va podalardagi hayvonlarning hattiharakati ham muhim ahamiyatga ega. Masalan, pingvinlar kuchli sovuqda bir joyga yig'ilib olib, aylanma harakatda bo'ladi. Vaqti-vaqti bilan eng chetdagi pingvinlar o'rtaga kirib joylarini almashtiradilar. Tuyalar jazirama issiq kunlari bir-birlariga yon boshlagan holda bir joyga to'planib yotadilar, ular to'plangan joy o'rtasidagi harorat Q390S ga, ya'ni tana haroratiga yaqin bo'ladi. Eng chekkadagi tuyalarning elka tomonidagi yunglari $\mathrm{Q}^{\circ}{ }^{\circ} \mathrm{S}$ gacha qizib ketadi. Bu paytda tuyalarning bo'taloqlari va boshqa qari vakillari to'da o'rtasidan joy olib himoyalanadi.

\section{Dunyo okeanining ekologik zonalari}

Okeanlar ekologik shart-sharoitlari bilan farqlanuvchi bir necha 
zonalardan tashkil topgan (4-rasm). Suvning yuza qatlami pelagial zona deb nomlanadi, tubi esa bental zonani tashkil etadi. Chuqurligiga qarab bental zonasi quruqlikning 200 metrga qadar pasayib borishi qismi - sublitoral, keskin chuqurlashish zonasida jadallashgan abissal zonalarga ajraladi. Okean suvining eng chuqur qatlami ultraabiassal zona hisoblanadi. Litoral zona suv to'lqini ko'tarilganda suv bilan ta'minlanib turadi. Uning yuqorisida supralitoral zona joylashgan va bu zona to'lqin qirg'oqqa urilganda, sachragan suv zarrachalari hisobiga namlanib turadi.

Okeanning har bir zonasida yashovchi hayvonlar o'ziga xos ekologik guruhlarni tashkil etadi. Jumladan, pelagial zonada pelagoslar tarqalgan, suvning yuzasida planktonlar bo'lsa, bental qatlamida bentos hayvonlar hayot kechiradi. Pelagoslar qish mavsumida qo'shimcha yorug'lik berilsa, u muddatidan oldin ko'payishi mumkin.

Plankton (lot. Planktos - sayr qiluvchi) - suv qatlamida yashab, suv oqimi bilan harakatlanuvchi organizmlar majmui. Plankton tarkibiga o'simliklar (fitoplankton), bakteriyalar (bakterioplankton), hayvonlar (zooplankton) kiradi. Ko'l planktoni limnoplankton, daryo planktoni potamoplankton deb ataladi. Fitoplankton suvning fotosintez qilish uchun zarur yuqori (50-100 m gacha) qatlamida, bakterio- va zooplankton suvning barcha qatlamida uchraydi. Dengiz fitoplaktoni, asosan, diatom suvo'tlar, peridiniyalar, kolitoforid xivchinlilardan, chuchuk suv fitoplaktoni diatom, ko'k yashil va ayrim yashil suvo'tlardan iborat. Chuchuk suv zooplanktonida kurakoyokli va shoxdor mo'ylovli qisqichbaqasimonlar, og'izaylangichlar, bir hujayralilar; dengiz zooplanktonida har xil qisqichbaqasimonlar, bir hujayralilar, bo'shliqichlilar, taroqlilar, baliqlar tuxumi va linchinkasi, umurtqasiz hayvonlar lichinkasi ko'p bo'ladi.

O’lchamiga binoan, plankton nannoplakton (bakteriyalar, juda mayda suvo'tlar), mikroplankton (suvo'tlar, bir hujayrali hayvonlar, og'izaylangichlar, har xil lichinkalar), mezoplankton (o'lchami 1 sm gacha bo'lgan qisqichbaqasimonlar va bentos hayvonlar), makroplanktonlar (o'lchami $1 \mathrm{sm}$ dan bir necha sm gacha bo'lgan mizidlar, krevetkalar, meduzalar), 
megaplankton (yirik hayvonlar, mas, qutb meduzasi - diametri $2 \mathrm{~m}$ gacha; zuxro kamari uz. 1,5 m gacha) ajratiladi. Ko'pchilik plankton organizmlarda suvda qalqib yurish uchun gavda nisbiy og'irligini kamaytiruvchi (havo pufaklari, yog' kiritmalari, g'ovak va liqildoq tana), gavda yuzasini kengaytiruvchi (gavdaning yassi bo'lishi, turli xil o'simtalar) moslanishlar paydo bo'lgan.

Okean fitoplanktoni umumiy massasi (550 mlrd. tonna) zooplanktonga nisbatan 10 marta ko'proq. Fitoplankton zooplankton uchun oziq, suv havzalaridagi oziq zanjirining asosini tashkil etadi.

Litoral (yun. lithoralis - qirg'oqqa tegishli) - dengiz sohilining suv bosib turadigan suv ko'tarilish-qaytish zonasi. Mo"tadil iqlim litoralida bir kechakunduz va yil davomida xarorat, suvning sho'rlanishi ancha o'zgarib turadi. Shu sababdan litoralda keng miqyosda moslanish xususiyatiga ega bo'lgan evribiont turlar: suvo'tlar (asosan, fukussimonlar, diatom suvo'tlar), qisqichbaqasimonlar (balanuslar, yonlab suzarlar), aktiniyalar, korall poliplar, mollyuskalar (midiya, littorina va Bentos) va Bentos organizmlar yashaydi. Qishda litoral muzlab qolishi mumkin, shuning uchun ayrim organizmlar sublitoralga ko'chib o'tadi, boshqalari o'zining faol hayotiy xususiyatlarini saqlab qoladi. Mo"tadil iqlimda $1 \mathrm{~m}^{2}$ sovuq litoralda 5-10 kg biomassa hosil bo'ladi. Arktika yoki Antarktida ta'sirida litoralda deyarli hayot bo'lmaydi. Chuchuk suvli ko'llarda litoral 5-7 m chuqurlikkacha hayotga eng boy zona hisoblanadi.

Nekton (grekcha "nektos" - suzib yuruvchi) - erkin suzuvchi va aralashib yuruvchi organizmlar. Bu baliqlar, amfibiyalar, hasharotlar.

Bentos (yun. Benthos - chuqurlik) - dengiz va chuchuk suv havzalari tubidagi balchikda va uning ustida yashaydigan organizmlar. Bentos o'simliklar (fitobentos) va hayvonlar (zoobentos) bentosiga ajratiladi.

Zoobentos o'z navbatida loyqada yashaydigan hayvonlar - infauna (ko'p tuklilar, ikki pallali mollyuskalar, exiuridlar, sipunkulidlar, ayrim ignaterililar va boshq)ga, loyqa ustida o'rmalab yuruvchilar - onfauna (ko'p tuklilar, mollyuskalar, ignaterililar, qisqichbaqasimonlar), substratga yopishib 
yashovchilar - epifauna (g'ovaktanlilar, gidroidlar, aktiniyalar, korallar, mshankalar, ayrim ikkipallalilar) suv tubi yaqinida suzib yuradigan, ba'zan suv tubiga tushib turadigan nektobentos (krevetkalar, mizidlar, ayrim goloturiyalar, suv tubi baliqlari va bentos)ga ajratiladi.

Tanasining o'lchami bo'yicha bentos makrobentos (5 - $10 \mathrm{~mm}$ va undan yirikroq organizmlar), mezobentos $(0.5 \mathrm{~mm}$ dan $10 \mathrm{~mm}$ gacha) va mikrobentosga (0.5 mm dan kichik) bo'linadi. Dengizning sayoz qismida fitobentos asosan makrofitlar (suv o'tlari)dan, chuqur qismida esa hayvonlar va bakteriyalardan iborat. Dengizlarda bentos biomassasi chuqurlik ortishi bilan kamayib boradi. Dengiz tubining 8\% ni tashkil etadigan sohil bo'yi suvlari (chuq. $200 \mathrm{~m}$ gacha) biomassasiga dengiz biomassasining 60\%, aksincha suv tubining 3G'4 qismiga teng bo'lgan abissal qismiga 10\% biomassa to'g'ri keladi. Okean bentos yalpi biomassasi 10-12 mlrd. t ga teng. Chuchuk suvlarda bentos son va turlar jihatidan nisbatan xilma-xil bo'lmaydi. Zoobentos tarkibiga har xil umurtqasiz hayvonlar, fitobentosga suv o'tlari va gulli o'simliklar kiradi. Bentos ko'pchilik baliqlar va bentos hayvonlar uchun oziq hisoblanadi.

Qushlarning juda ko'pchiligi tirikligini, asosan, kunduzi o'tkazadigan hayvonlar bo'lib, kun botishi bilan tinadi.

Qushlar hayotidagi mavsumiylik ularning safar uchishlari bilan bog'liq. Masalan, qaldirg'ochlar qishlashi uchun Janubiy Afrikaga uchib boradi. Qushlar safar uchishlarida Quyoshga, Oy va yulduzlarga qarab mo'ljal oladi. Bunda ular galalar yoki yakka-yakka holda uchadilar. Qushlarning kelibketishi tug'ma instinktiv hodisa. Masalan, kakku qushlarning voyaga etgan bolalari ota-onalaridan oldin uchib ketadi va etar manzilini aniq mo'ljalga oladi. Chumolining tundagi harakatida Oy yoritgich va mo'ljal sifatida ahamiyatga ega. Hozir faqat Quyosh, Oy, yulduzlar emas, Erning magnit maydoniga qarab mo'ljal olish ilmiy tomondan asoslandi.

Dengiz va okean suvlari tagida yashaydigan ko'pchilik mavjudotlar, quruqlikda yashaydigan hasharotlar o'zlaridan nur taratish xususiyatiga ega. $\mathrm{Bu}$ hodisa biolyuminestsentsiya deb ataladi. Bunday xususiyat bakteriyalardan 
tortib baliqlargacha kuzatiladi. Hayvonlarning o'lja topishida, himoyalanishi va ko'payishida biolyuminestsentsiyaning ahamiyati beqiyos.

\section{Suv muhitidagi hayot}

Suv muhiti yuqori darajadagi zichligi, bosimning keskin o'zgarishi, kislorodning kamligi, quyosh nurining jadal yutilishi va boshqa xususiyatlari bilan ajralib turadi. Suv havzalarining har bir joyi o'ziga xos tuz va zarrachalar tarkibi, oqim kuchiga ega.

Suv tubida yashovchi organizmlar uchun esa tuproqning xossalari, undagi organik qoldiq ulushi muhim ahamiyatga ega. Hayvonlar suv muhitining umumiy xususiyatlaridan tashqari undagi xususiy omillarga ham moslanishlari zarur. Suv muhitida yashovchi barcha organizmlar gidrobiontlar deb nomlanadi. Suvning yuza qatlamida yashovchi organizmlar esa planktonlar va tubida yashovchilar bentoslar ekologik guruhlarini tashkil etadi. Mazkur ekologik guruh vakillari dunyo okeanida, dengizlarda, ko'llarda, ichki suv havzalarida va er osti suvlarida tarqalgan.

Suv muhiti hayotini belgilovchi omillardan eng muhimi suvning bosimidir. Chuqurlikning ortishi bilan bosim kuchi ortib boradi. Masalan, har $10 \mathrm{~m}$ chuqurlikda bosim kuchi 1 atmosferaga ortadi. Suv muhitidagi bosim kuchining o'zgaruvchanligi tufayli aksariyat gidrobiontlar evribiontlardir. Ular ayrim bir necha atmosferadan yuz atmosferaga qadar bo'lgan bosim sharoitida yashashga moslashgan. Jumladan, Elpidia goloturiyalari, Priapulus caudatus chuvalchanglari okeanlarning qirg'oqlaridan ultra abissal zonalariga qadar uchrashi mumkin. Chuchuk suvlarda yashovchi infuzoriya tufelkalari, suvoykalar ham tajribada 600 atmosfera sharoitiga bardosh bergan. Lekin gidrobiontlarning ko'pchiligi stenobatlar sifatida dengiz va okeanlarning ma'lum chuqurliklaridagina yashashga moslashgan. Masalan, ayrim baliqlar, bosh oyoqli mollyuskalar, qisqichbaqasimonlar, pogonoforalar, dengiz yulduzlari suvning chuqur qatlamlarida 400-500 atmosferadan kam bo'lmagan sharoitda yashaydi. Umuman, stenobatlar kichik suv havzalarida va suvning chuqur qatlamlarida uchraydi.

Suv muhitidagi zichlikning yuqoriligi gidrobiontlar uchun tayanch ahamiyatiga ega. Shuning uchun ham ko'pchilik hayvonlar suvning yuza qatlamida nisbatan past bosimda, doimiy harorat va etarli yorug'lik sharoitida yashaydi. Bentoslar esa yuqori bosimga, past haroratga va doimiy 
qorong'ulikka moslashgan hayvonlardir.

Pelagial hayvonlar. Pelagial qatlam vertikal yo'nalish bo'yicha farqlanuvchi epipelagial, batipelagial va abiss pelagial zonalarga farqlanadi. Quyosh nuri epipelagialning quyi chegarasiga qarab etib boradi, mazkur zonadan keyingi chuqurliklarda yashil o'simliklar uchramaydi.

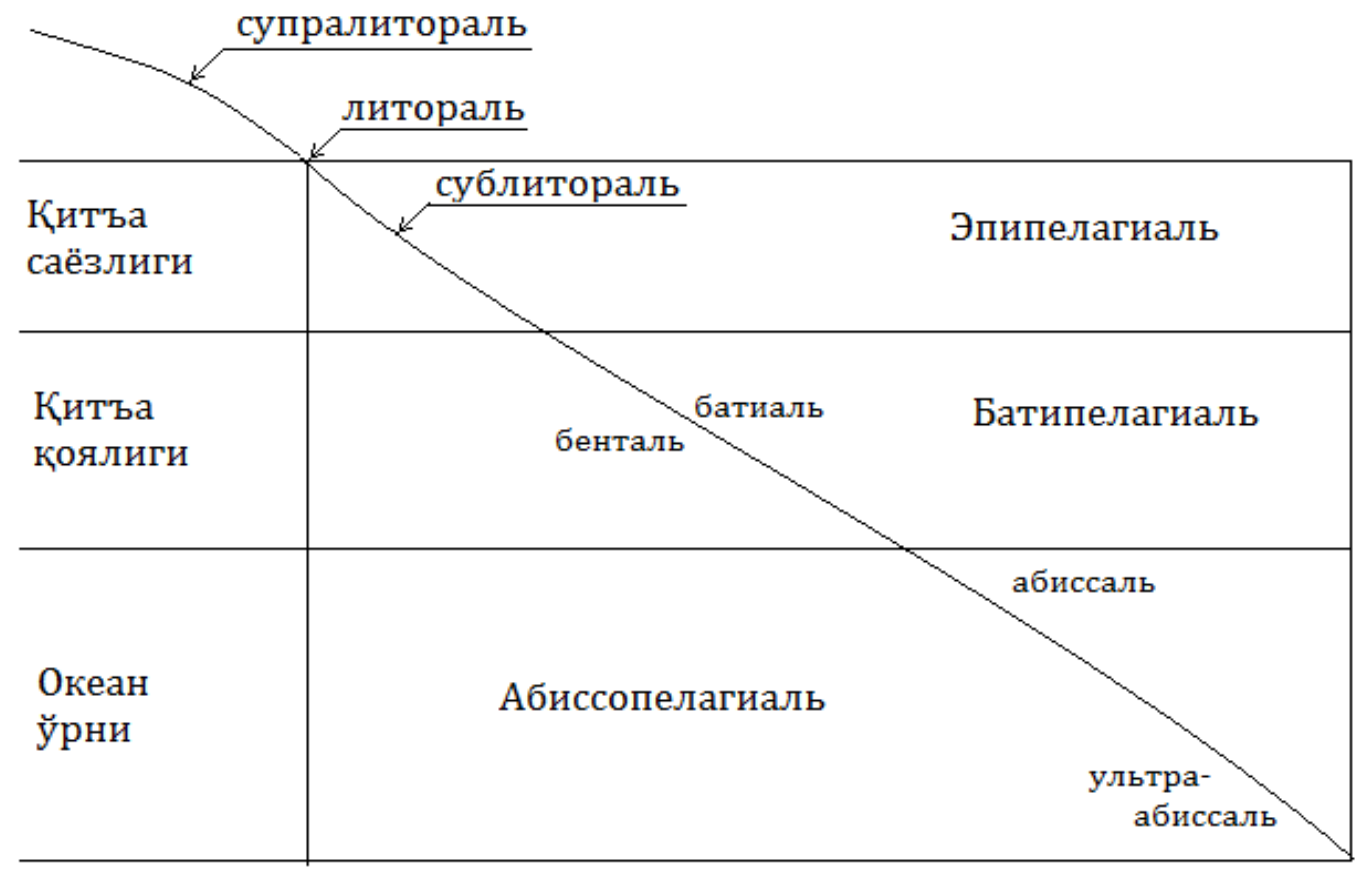

4-rasm. Dunyo okeanining ekologik zonalari

Yuqorida ta'kidlangan ekologik zonalar faqatgina okeanlar uchungina xos bo'lmay, boshqa suv havzalarida ham qayd etilgan.

\section{Gidrobiontlarning ba'zi bir moslanishlari}

Suv muhiti turli-tuman va o'ziga xos ekologik sharoitlarga ega. Shuning uchun gidrobiontlarda suv muhitida mo'ljal olish, filtrlash yo'li bilan oziqlanish, vaqtli suv havzalarida yashashga moslanish kabi va boshqa morfoekologik ixtisoslanishlar vujudga kelgan.

Hayvonlarning suv muhitida mo'ljal olishi. Suv tubidagi doimiy qorong'ulik va yorug'lik nurining jadal so'nib borishi hayvonlarni ko'z orqali mo'ljal olish imkoniyatini keskin chegaralaydi. Ma'lumki, suvda tovush havo muhitiga nisbatan tez tarqaladi. Shuning uchun ko'z yordamida mo'ljal olishdan ko'ra gidrobiontlar uchun tovush yordamida mo'ljal olish bir muncha qulayroq hisoblanadi. Hayvonlar o'ta nozik to'lqin uzunliklari o'zgarishlarini 
seza oladi. Masalan, meduzalar po'rtana boshlanishidan ogoh etuvchi infratovushlarni sezgirlik bilan ilg'ab suv yuzasidan chuqur qatlamlarga tushib ketadi. Baliqlar, mollyuskalar qisqichbaqasimonlar va sut emizuvchilar tovush chiqarish orqali aloqada bo'ladi. Masalan, qisqichbaqasimonlar tanasining turli qismlarini bir-biriga ishqalash orqali, baliqlar havo pufaklari, halqum tishlari, jag'lari, ko'krak suzgichlari yordamida turli tovushlarni chiqaradi. Tovushlar tur ichidagi munosabatlarni muvafiqlashtirish, galadagi aloqani ta'minlash ikkinchi jinsni o'ziga jalb etish, ogohlantirish yo'llaridan biridir.

Ba'zi bir gidrobiontlar ozuqa topishda aks sadodan unumli foydalanadi (masalan, kitsimonlar). Ayrim gidrobiontlarning elektr impulslarini ishlab chiqish va qabul qilish xususiyati ham ma'lum. Hozirda 300 turga yaqin baliqlar elektr zaryadlari orqali mo'ljalni aniq oladi, o'ljaga hamla qilishda va himoyalanishda undan unumli foydalanadi.

Suvdagi gidrostatik bosimning o'zgarishi hayvonlar havo pufaklari, statotsistlari va boshqa organlari yordamida aniq farqlay oladi.

Barcha gidrobiontlarda xemoretseptorlar yaxshi taraqqiy etgan. Shu sababdan ular suv muhitida yuz bergan barcha kimyoviy o'zgarishlarni osonlik bilan payqay oladi. Ayniqsa minglab km. masofa bo'ylab migratsiya qiluvchi baliqlar hidni yaxshi farqlaydi. Masalan, urchish uchun Evropaning barcha daryolaridan atlantika okeani tropik qismining g'arbiy hududlariga boradigan daryo ugorlari tajribada $6000 \mathrm{~km}^{3}$ suvda erigan $1 \mathrm{~g}$ etil spirtini ham sezgan.

Filtrlash yo'li bilan oziqlanish. Gidrobiontlarning ayrimlari suvdagi mayda organik zarrachalarni suzib oziqlanadi. Filtrlash yo'li bilan oziqlanish plastinka jabrali mollyuskalar, o'troq hayot kechiruvchi ninaterililar, polixetalar, astsidiyalar uchun xos usuldir. Bu guruhga mansub organizmlar oziqlanish jarayonida suvni filtrlab muhim biologik vazifani ham amalga oshiradi.

Gidrobiontlarning vaqtli suv havzalarida yashashga moslanish xususiyatlari. Tabiatda mavsumiy hosil bo'ladigan vaqtli va ko'lmak suv havzalari ko'p uchraydi. Qisqa muddat bo'lsada, ularda qator gidrobiontlar hayot kechiradi, ko'payadi va rivojlanadi. Bu hayvonlar suv havzasi qurib qolganidan so'ng keladigan uzoq muddatli qirg'oqchilikka bardosh beradi. Suv 
etarli bo'lganda gidrobiontlar jadal ko'payadi va mo'l nasl beradi, noqulay sharoitda ularning ko'pchiligi gipobioz holatga o'tadi. Masalan, qolqondorlar, shoxdor mo'ylovli qisqichbaqalar, planariyalar, kamtukli chuvalchanglar, mollyuskalar, baliqlardan - protepterus, shoxtish va lepidosirenlar va boshqalar. Suvsizlik sharoitida quyoshsimonlar, infuzoriyalar, ildizoyoqlilar, ayrim kurakoyoqli qisqichbaqasimonlar va boshqa hayvonlar maxsus tsistaga o'ralab oladi va o'z hayotini saqlab qoladi.

Noqulay sharoitda kolovratkalar va nematodalarning ayrim vakillari tanasidagi namlikni butunlay yo'qotib yupqa plyonka shaklini oladi, qulay sharoitda "jonlanib" yana hayotini davom ettiradi.

\section{Hayvonlarning quruqlik - havo muhitida yashashga moslanishlari}

Evolyutsiya jarayonida quruqlik va havo muhiti hayvonlar tomonidan suv muhitiga nisbatan bir muncha kechroq o'zlashtirilgan. Quruqlik - havo muhitidagi hayot hayvonlardan ancha takomillashgan va murakkab moslanishlarni talab etadi. Mazkur muhitda organizmlar doimo turli gazlar qurshovida bo'ladi, yuqori kontsentratsiyadagi kislorod, past zichlik va kam miqdordagi suv bug'lari hayvonlarning hayotiy jarayonlariga kuchli ta'sir etadi. Past zichlik tufayli havoning ko'tarish kuchi va tayanch ahamiyati bir muncha chegaralangan. Shuning uchun havo muhitida yashashga moslashgan hayvonlarda xususiy tayanch tizimini ta'minlovchi mustahkam skelet shakllangan. Havo muhiti ko'tarish kuchining sustligi quruqlikdagi hayvonlarning o'lchami va og'irligini chegaralab qo'ygan. Quruqlikda suvdagi kabi gigant hayvonlar uchramaydi. Agar kitlar kattaligidagi hayvonlar quruqlikda yashaganda, ular o'z og'irliklari hisobiga nobud bo'lishi mumkin edi.

Havodagi past zichlik uning qarshilik kuchini susaytiradi va hayvonlarning harakatlanishi uchun qulay imkoniyat yaratadi. Evolyutsiya jarayonida aksariyat hayvonlar ushbu ekologik qulaylikdan samarali foydalanishga ixtisoslashgan. Jumladan, quruqlik - havo muhiti hayvonlarning 75\% (hasharotlar, qushlar, sudralib yuruvchilar, sut emizuvchilar) havoda uchishga moslashgan. Ko'pchilik hasharotlar, sodda hayvonlarning tsistalari havoda passiv holda uchib yuradi. Bu hodisa anemoxoriya deyiladi. Havo 
oqimi yordamida passiv holda uchib yuruvchi barcha organizmlar aeroplanktonlar deb nomlanadi. Aeroplanktonlar havo muhitiga tanasining kichik o'lchamda bo'lishi, har xil o'simtalar hisobiga tana yuzasini kengaytirishi, kuchli darajada bo'laklarga ajralishi, qanotlarning nisbatan keng yuzaga ega bo'lishi, o'rgimchak ipi tolasi kabilardan foydalanish bilan moslashgan. Mikroorganizmlar va ayrim hayvonlarning tarqalishida havo oqimi va kuchsiz shamollar asosiy ahamiyatga ega. Kuchli shamol va to'fonlar ham hayvonlarga ta'sir etadi. Ba'zi bir mayda hayvonlar, qushlar va hasharotlar kuchli shamol (havo oqimi)ga bardosh bera olmaydilar. Okean orollarida yashaydigan hasharotlarning ichida ko'pincha qanotsiz formalar uchraydi, qushlarda esa ingichka uzun qanotlar shakllangan. Qanotsiz hasharotlar shamol ta'siridan himoyalanish maqsadida toshlar ostiga, qoya yoriqlariga yoki o'simlik qoplamlari ichkarisiga yashirinib oladi.

Quruqlikdagi bosim dengiz sathidan yuqoriga ko'tarilgan sari, vertikalmintaqalar bo'ylab kamayib boradi. Natijada ko'pchilik hayvonlarning tog'lardagi tarqalishi $6000 \mathrm{~m}$ balandlikka qadar chegaralangan.

Havo organizmlar uchun yashash muhitigina bo'lmasdan, balki ekologik omil sifatida ham ahamiyatli. Atmosferadagi havo tarkibida 78,1 \% azot, 21\% kislorod, 0,9\% argon, 0,03\% karbonat angidrid gazlari mavjud. Bulardan tashqari, oz miqdorda neon, geliy, kripton, ksenon, ammiak, vodorod gazlari, radiy, toriy kabi radioaktiv moddalar qoldiqlari, shuningdek azot va oltingugurt oksidlari, xlor va boshqa elementlar uchraydi. Havodagi suv bug'larining ulushi $0,01-4 \%$ ga qadar bo'ladi. Havoning quyi qatlamlaridagi gazlar tarkibida tabiiy aralashmalar ham uchraydi. Ular chang va tutunlar, qurum, dengiz tuzlarining kristallari, har xil organik zarrachalar bo'lishi mumkin. Havo tarkibidagi kislorod hayvonlarning nafas olishi uchun eng zarur elementdir. Kislorod hisobiga quruqlik organizmlaridagi nafas olish va moddalar almashinuvi jarayoni jadallashgan va gomoyotermlik xususiyati shakllangan. Havo tarkibidagi kislorod hayvonlar uchun etarli darajada bo'ladi. Agar uning havodagi ko'rsatkichi 5\% ga kamaysa, organizmlarning nafas olishi qiyinlashadi. Karbonat angidrid gazining miqdori ortiq bo'ladi. Havoning oltingugurt va azot oksidlari, galogenlarning vodorodli birikmalari va boshqalar zararli moddalar bo'lib, uning ifloslanishiga sabab bo'lmoqda. $\mathrm{Bu}$ holat tirik organizmlarga salbiy ta'sir etadi. 
Namlikning etishmasligi quruqlik - havo muhitining o'ziga xos xususiyatlaridan biri. Shuning uchun quruqlik organizmlardagi morfoekologik moslanishlarning barchasi namlikni ta'minlash va saqlab qolishga yordam beradi. Quruqlik - havo muhitida haroratning o'zgarish chegarasi ancha katta bo'ladi. Masalan, Markaziy Osiyo cho'llari sharoitida haroratning tebranishi 68-770 S ni, o'rtacha kunlik ko'rsatkichi esa $25-38^{\circ} \mathrm{S}$ ni tashkil etadi. Muayyan ekologik sharoitda namlik, harorat va yorug'likka hayvonlarning adaptatsiyalanish xususiyatlari abiotik omillar mavzularida batafsil yoritilgan.

Hayvonlarning tarqalishi, migratsiyasi va yashash joylarini tanlashida joyning rel'efi ma'lum ahamiyatga ega. Jumladan, tuyoqli hayvonlarni va tuyaqushlarni erkin harakati uchun zich tuproqli mintaqalar qulay bo'ladi. Qum uyumlarida va ko'chma qumlarda yashaydigan sudralib yuruvchilarning panjalari keng joylashgan katta muguzli yuzaga ega bo'ladi va bu holat harakatlanishni osonlashtiradi. Erni kovlab yashovchi hayvonlar odatda mayin tuproqli qiyaliklarni tanlaydi.

Yog'inlar (yomg'ir, do'l, qor) suv bilan ta'minlash bilan hayvonlar hayotida ma'lum ekologik ahamiyatga ham ega. Kuchli yoqqan yomg'ir va do'llar hayvonlarga mexanik ta'sir etadi. Yomg'ir tuproqni yuvib hasharotlar g'umbaklarini ochib qo'yadi, quyosh nuri ta'sirida ular qurib nobud bo'ladi. Shiralar yomg'ir bilan yuvilib erga tushadi, tuproq va loyga qorishib qirilib ketadi.

Qor qoplami hayvonlar uchun fon va substrat sifatida ahamiyatga ega. Hayvonlar qor qoplami bo'ylab harakatlanadi, uning ostida yashaydi, ozuqa to'playdi va himoyalanadi. Qor qoplami bo'ylab hayot kechiruvchi hayvonlar xionofoblar deb nomlanadi, qor ostida yashovchilar - xionofillar ekologik guruhini tashkil etadi, ularga qur va qarqurlar misol bo'ladi. Xionofoblar ular o'rtasidagi oraliq holatni egallaydi. Xionofoblarning rangi fonga mos holda oq bo'ladi. Masalan, oq tulkilar, lemminglar, oq quyon, oq kuropatka va boshqalar. Qor qisqa muddat saqlanadigan hududlarda tarqalgan hayvonlarning rangi deyarli o'zgarmaydi. Qor qoplami hayvonning harakatlanishi va ovqat topishiga bir muncha qiyinchilik tug'diradi. Masalan, 40-50 sm qalinlikdagi qorda shimol bug'ulari bemalol harakatlana oladi, qulonlar va antilopalar esa o'rnidan siljiy olmaydi. Qorda erkin harakat qiladigan hayvonlarning panja va tuyoqlari yuzasi ancha keng bo'ladi. Qish 
mavsumiga tomon tuyoqlar va panjalar yuzasi kengayib atrofidagi dag'al yunglarning uzunligi va zichligi ortadi. Qorning qalinlashishi va muzlamalar xionofoblarning ovqat topishini qiyinlashtiradi. Masalan, qoramollar 20-30 sm qor tagidagi o't-o'lanlarni topib eydi. Qo'y va echkilar - 10-15 sm, bug'ular esa $40 \mathrm{sm}$ li qor qoplamini buzib ozuqasini topa oladi.

Qor tushishi bilan ko'pchilik hayvonlar yashash joylarini o'zgartiradi. Yuqori tog' mintaqalarida tarqalgan hayvonlar qishda quyi mintaqalar tomon siljib, pastga tusha boshlaydi. Qish oylarida yirtqichlarning oziqlanish spektrida sezilarli o'zgarish ro'y beradi. Ular asosan xionofoblarni ovlaydi. Qalin qor va ayozli muzlamalar hayvonlarga salbiy ta'sir etadi, natijada ular ko'plab qirilib ketadi. Xionofoblar qalin qor uzoq saqlanadigan sharoitda ham bemalol yashay oladi. Jumladan, quyonlar qalin qorda daraxtlarning po'stlog'i butalarning uchki novdalari bilan ovqatlansa, tovushqonlar o'tlar bilan oziqlanadi. Qor kuchayib o'simliklarni ko'mib qo'ysa, quyonlar ochlikdan qiynaladi.

Mayda kemiruvchilar - xionofillar qor ostida uya yasab hayot kechiradi. Qor qoplami ularni sovuqdan va dushmanlaridan himoya qiladi. Qulay sharoitda xionofillar qish mavsumi davomida bemalol ko'payib avlod beradi. Qor kam yog'ib tuproq yuzasi muzlasa, ular uchun noqulay sharoit yuzaga keladi va ko'pchilik xeonofillar qirilib ketadi.

Qurlar, chillar va oq kuropatkalar ayozli kunlarda va kechalari qor ichiga kirib yashirinadi. Chunki havo harorati $-40^{\circ} \mathrm{S}$ ga qadar pasayganda ham, qor orasidagi harorat $-10^{\circ} \mathrm{S}$ dan pastga tushmaydi.

\section{Tuproqning muhit sifatida ahamiyati}

Tuproq quruqlikning havo bilan bog'langan yupqa qatlami bo'lib, organizmlarning tarqalishida va hayotida muhim ahamiyatga ega. Tuproq qattiq zarrachalarning gazlar va suv bilan bog'langan holdagi o'ziga xos tizimi bo'lib, unda xilma-xil ekologik sharoitlar shakllangan.

Tuproqda mo"tadil harorat va etarli namlik saqlanadi. Organizmlarning qoldiqlari parchalanishi bilan organik massa zahirasi boyib boradi. Tuproqning muhit sifatida organizm uchun ko'rsatadigan asosiy xususiyatlariga uning tarkibi, moddalarning davra bo'ylab aylanishi, ya'ni 
gazlar, suv, organik, mineral moddalarning o'zlashtiriladigan holiga aylanishi va boshqalar kiradi. Shu bilan birga, tuproqda yashovchi barcha organizmlar uning tarkibiy va boshqa o'zgarishlariga kuchli ta'sir etadi.

Tuproqda o'simliklar, mikroorganizmlar, umurtqasiz va umurtqali hayvonlar hayot kechiradi. Ular uchun tuproq qatlamining chuqurligi, zarrachalarning o'lchami katta ekologik ahamiyatga ega. Bulardan tashqari, tuproq harorati, namligi, aeratsiyasi, unda yashovchi joylari va shartsharoitlari bilan farqlanuvchi mikrofauna, mezofauna, makrofauna va megafauna ekologik guruhlarga ajratiladi.

Mikrofauna $(0,002-0,2 \mathrm{~mm})$ vakillari - xivchinlilar, soxta oyoqlilar, infuzoriyalar, kolovratkalar, nematodalar uchun tuproq mikro suv havza vazifasini bajaradi. Mikrofaunaning barchasi suv hayvonlari hisoblanadi. Tuproqda bu jonivorlar suv bilan to'lgan tuproq bo'shliqlarida hayot kechiradi. Mikrofaunaning qator vakillari ochiq suv havzalarida ham tarqalgan. Tuproqda yashovchilarning o'lchamlari chuchuk suvdagilarga nisbatan bir muncha mayda bo'ladi. Shuningdek, ular uzoq muddatli tinim davrida bo'lishi mumkin.

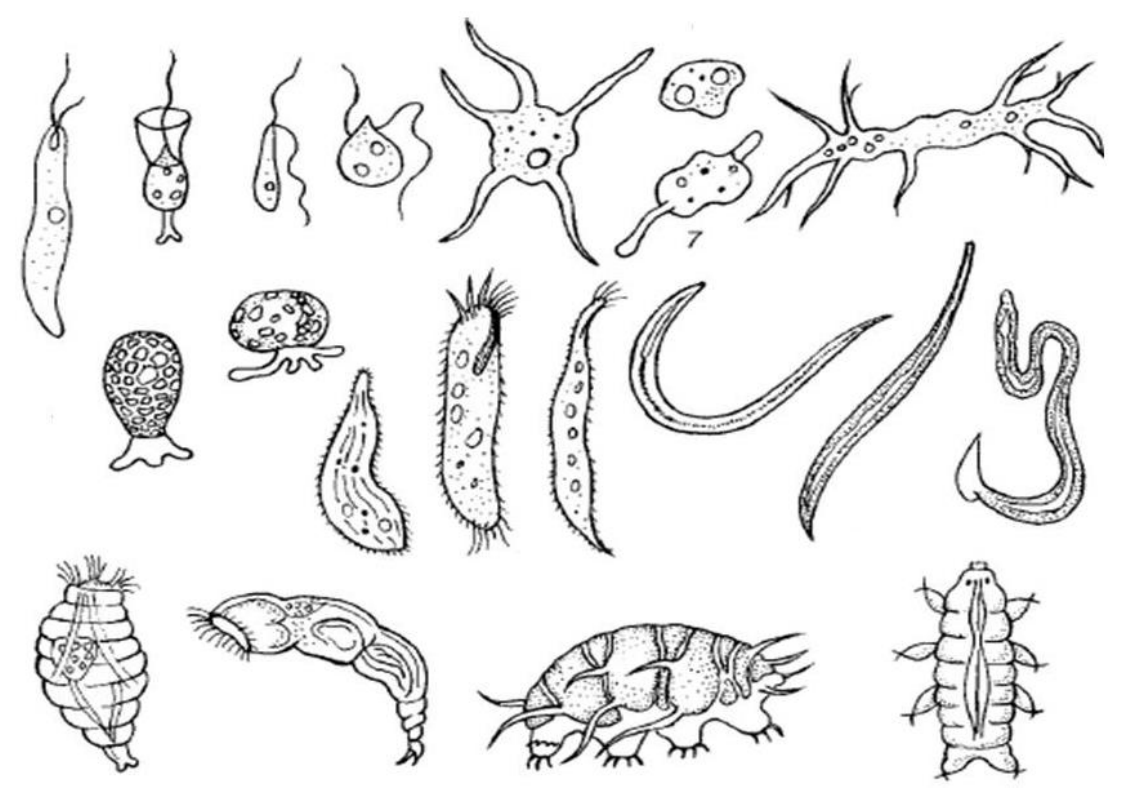

5-rasm. Tuproq mikrofaunasi

Havodan nafas oluvchi yirikroq hayvonlar uchun tuproq bo'shliqlari "g'or" vazifasini bajaradi. Tuproq "g'or" larida o'lchamli 0,2-3 mm bo'lgan mezofauna vakillari uchraydi. Kanalar, birlamchi qanotsiz hasharotlar, qanotli hasharotlarning mayda vakillari, ko'poyoqlilar, simfillalar va boshqalar 
mezofaunaga mansub organizmlar. Ular tuproq bo'shliqlari bo'ylab o'rmalab yoki chuvalchangsimon harakatlanib hayot kechiradi va tanasi orqali havoga to'yingan suv bug'lari vositasida nafas oladi. Mezofauna vakillarining ko'pchiligida traxeyalar tizimi yo'q. Shu sababdan ular suvsizlikka chidamsiz. Namlik kamayishi bilan ular tuproqning chuqurroq qatlamlariga siljib yashash joylarini o'zgartiradi.

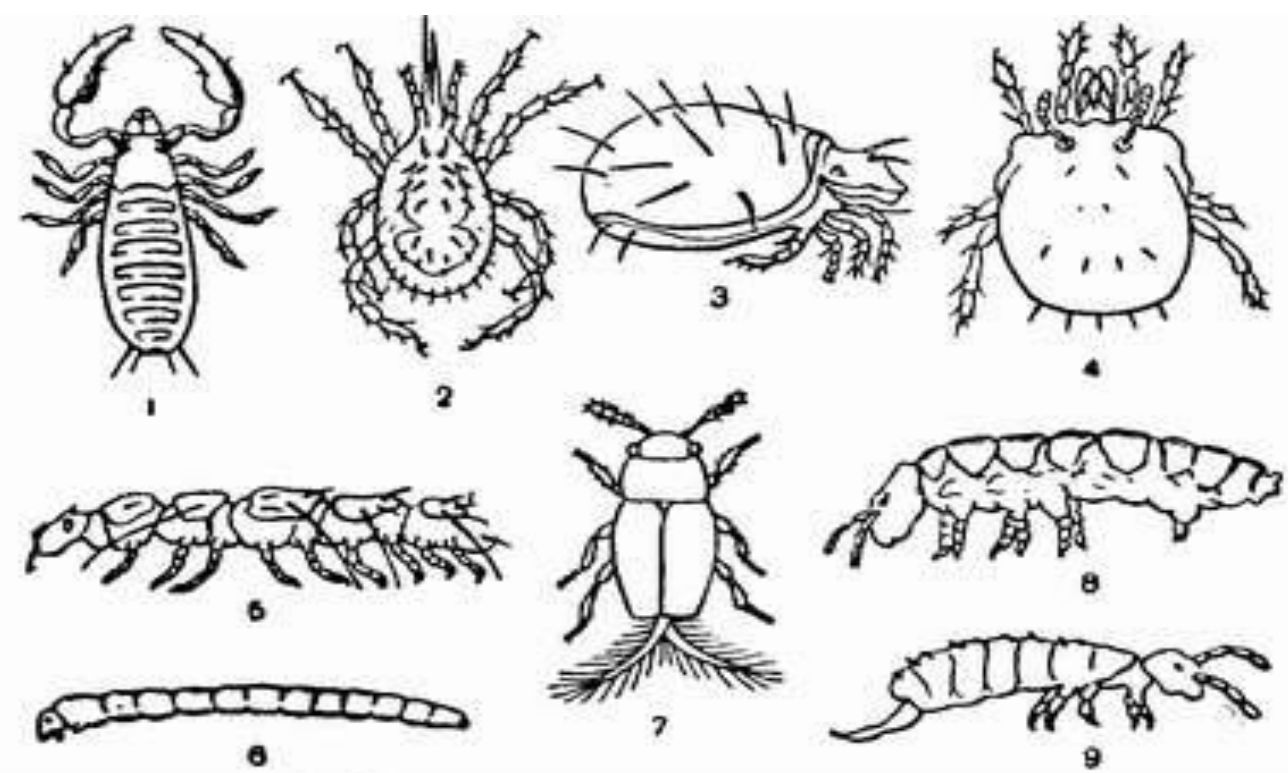

6-rasm. Tuproq mezofaunasi

Mezofaunaning yirikroq vakillari tanasi qattiq xitin qobig'i yoki tangachalar bilan himoyalangan bo'lib, noqulay sharoitdan saqlaydi. Mikro va mezofauna vakillari tuproqning muzlashiga bardoshli bo'ladi.

Tana o'lchami 2-20 mm bo'lgan hayvonlar makrofauna ekologik guruhini tashkil etadi. Hasharotlar lichinkalari, ko'poyoqlilar, yomg'ir chuvalchanglari uchun tuproq zich muhit bo'lib, ularning harakatlanishiga mexanik jihatdan qarshilik qiladi. Shuning uchun ular tuproqning surib yoki o'ziga yo'l ochib harakatlanadi. Ular tanasining ko'ndalang kesimi nisbatan kichik, oson egilish va bukilish xususiyatiga ega. Tuproq zarrachalarini bunday hayvonlar tanasining bosimi yordamida surib qo'yishi mumkin. Bosh va dum qismlari suyrilashgan, tanasi orqali cho'zilish xususiyatiga ega. Makrofauna vakillari maxsus nafas olish organlari orqali yoki butun tanasi bo'ylab nafas oladi. Noqulay sharoitda va qish mavsumida ular tuproqning chuqur qatlamlariga yig'ilib himoyalanadi. 


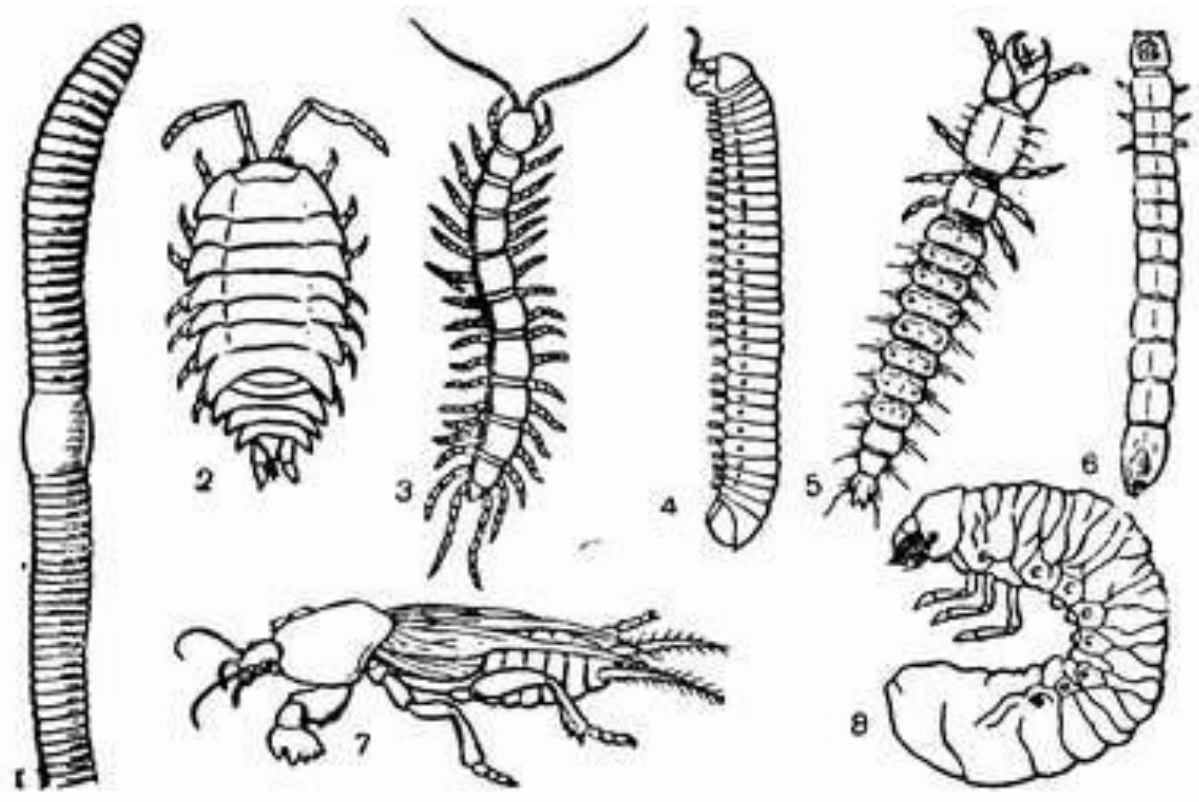

7-rasm. Tuproq makrofaunasi

Tuproqni kavlab hayot kechirishga ixtisoslashgan ko'rsichqonlar, krotsimon ko'rsichqonlar, krotlar, so'qir sichqonlar, tillarang krotlar, xaltali krotlar va boshqalar megafaunaning vakillari hisoblanadi. Ularning butun hayoti er ostida o'tadi. Gavdasining tuzilishi ham er ostida hayot kechirishga, er qazib, er osti yo'llarini yasashga moslashgan. Barchasining gavdasi o'qloqsimon, bo'yinlari kalta muloyim junlari kalta va qalin bo'lib, tuproq va namni o'tkazmaydi. Oyoqlari qisqa va kuraksimon shaklda bo'lib, erni kavlashga yaxshi moslashgan. Ko'zlari rivojlanmagan kichik o'lchamda, osilgan qovoqlari ularni ustidan yopib turadi. Megofaunaning boshqa vakillaridan farqliroq ko'r sichqonlar kurak tishlari yordamida erni kavlab murakkab, er osti yo'llarini yasaydi.

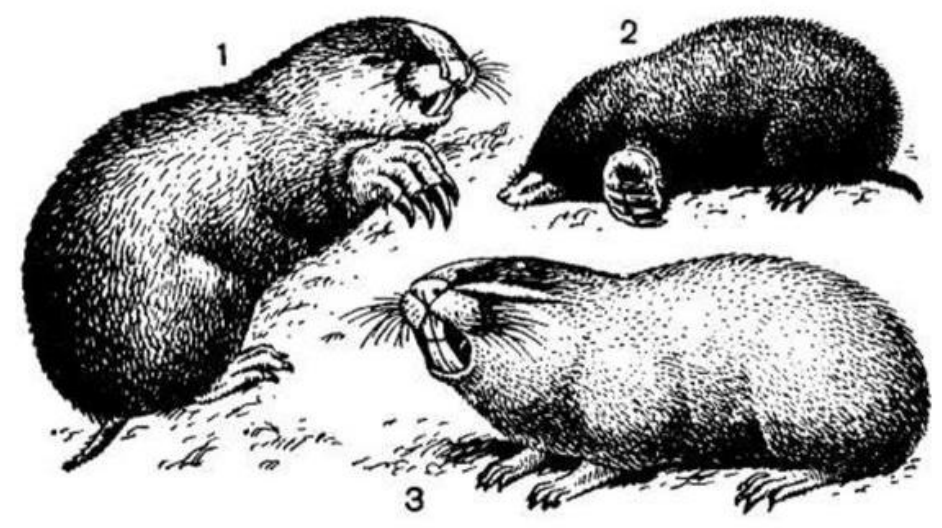

8-rasm. Tuproq megafunasi

Janubiy yarim shar tropik o'rmonlarida yashaydigan yirik 2,5 - 3 metr 
uzunlikdagi oligaxet chuvalchanglari ham megafauna tarkibiga kiradi. Tuproqda, yuqorida ko'rib o'tilgan ekologik guruhlardan tashqari uya (in) qurib yashovchi hayvonlar guruhi ham farqlanadi. Jumladan, yumronqoziqlar, sug'urlar, qo'shoyoqlar, bo'rsiqlar, quyonlar va boshqalar tuproq ostida ko'payadi, dushmanlardan himoyalanadi, qishlaydi va qisqa muddatli dam oladi. Ular o'zlarining er kovlashga moslashgan oyoqlarini yaxshi rivojlanganligi, bosh qismining kichik o'lchamliligi, o'ziga xos gavda tuzilishi va boshqa moslanishlari bilan ajralib turadi.

Umuman, tuproq ekologik muhit sifatida hayvonlar evolyutsiyasida alohida ahamiyatga ega bo'ladi. O'zining tarixiy taraqqiyoti ko'pchilik bo'g'imoyoqlilar quruqlikka suv - tuproq - quruqlik yo'nalishi bo'ylab chiqqanligi ma'lum.

\section{Biotik omillar}

Tabiatdagi biotik munosabatlar xilma-xil bo'lib, ularning eng muhimlari yirtqich-o'lja, parazit-xo'jayin, tekinxo'rlik, o'zaro foydali, qarama-qarshi aloqalar, betaraflik, o'simlik va hayvonlar o'rtasidagi o'zaro aloqalar, sig'indilik, uya parazitizmi va boshqalar hisoblanadi. Bularning barchasi uyg'unlikda biotik omillarni tashkil etadi.

Yirtqich-o'lja va parazit-xo'jayin munosabatlari organizmlar o'rtasidagi ozuqa orqali bo'ladigan munosabatlardir. Chunki, geterotroflar boshqa geterotrof yoki avtotroflar hisobiga yashaydi. Ma'lumki, yirtqichlar o'ljasini ushlab jonsizlantirib, so'ng eydi. Shuning uchun yirtqichlarda o'ziga xos fe'latvor (aktiv reaktsiya, tez harakat va boshqalar) shakllangan bo'ladi. Mushuksimonlar oilasiga mansub bo'lgan gepardlar o'lja ketidan quvlaganda, bir sekundda 20 metr yo'lni bosadi, soatiga 110 hatto $140 \mathrm{~km}$ tezlikda chopa oladi. 


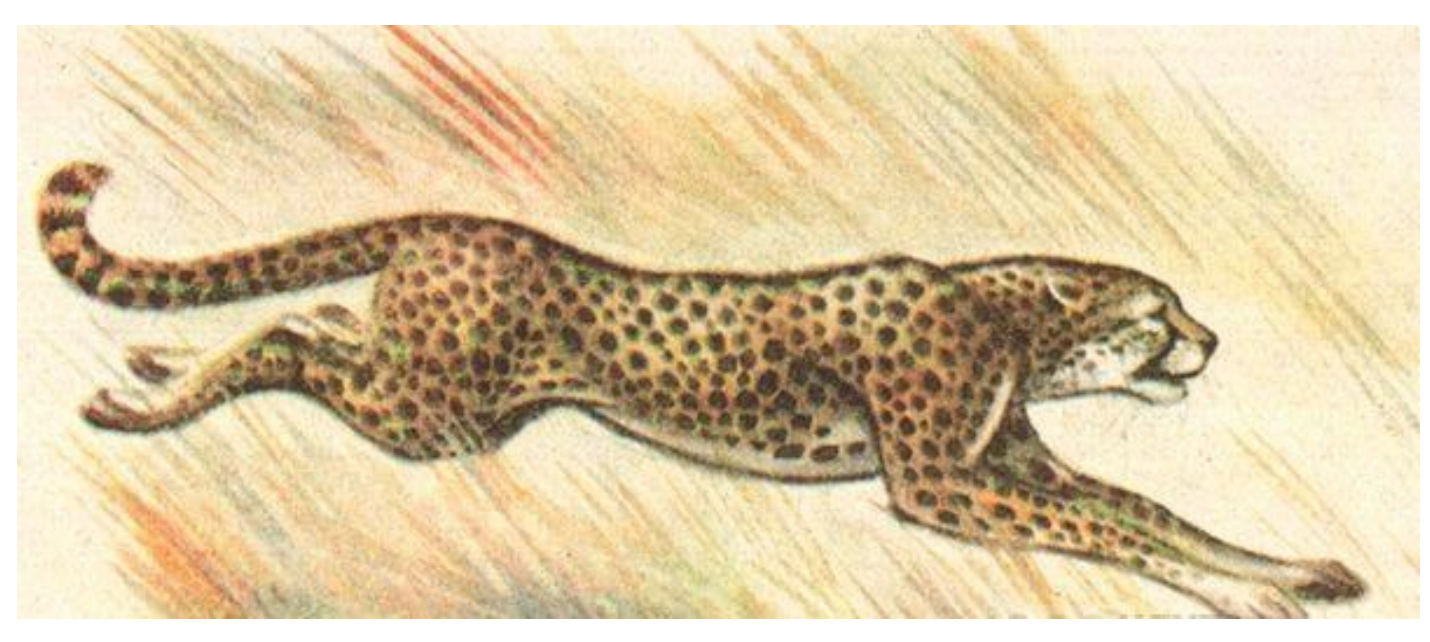

9-rasm. O’ljasini tutishga otlangan gepard

Yirtqichlar o'z o'ljasini ta'qib qilish va ularni ushlash paytida muayyan energiya sarf etadi va ma'lum qarshilikka ham uchraydi. Bu esa, yirtqich o'ljada o'ziga xos ekologik moslanishning shakllanishi va ayni organizmlar guruhining progressiv evolyutsiyalanishiga sabab bo'ladi. Shu bilan birga, o'ljada passiv himoya vositalari - bilintirmay qo'yadigan aks-soya, chalg'ituvchi va ogohlantiruvchi rang, narsalarga va boshqalarga tahdid qilish, himoya qalqoni, tikanlar va boshqalar vujudga kelgan. Bularning barchasi tabiiy tanlashning natijasi hisoblanadi.

Yirtqichlarda o'ziga xos moslanishning belgisi - ularning keng ozuqa spektriga ega ekanligidir. Buning asosida yirtqichlar alohida bir o'ljaga bog'lanib qolmasdan, sharoitga qarab osonlikcha bir o'ljadan ikkinchisiga o'ta oladi. Tabiatda yirtqichlar ovqatlanishi uchun imkoni boricha kam energiya sarf etishga harakat qiladi. Shuning uchun ham bular son jihatdan ko'proq bo'lgan va oson ovqatlanadigan, lekin biologik to'la qiymatli o'lja bilan oziqlanadi. Oq ayiqlar xilma-xil jonivorlar bilan oziqlanadi: tyulenlar, baliqlar, lemminglar (sichqonlar oilasiga mansub tur), oq tulki va boshqalarni ovlaydi. Lekin bularning ichida u tyulenlar go'shtini xush ko'radi, ilvirslar esa echkilarni bir necha soatlab poylab yotadi.

Yaqin vaqtlarga qadar yirtqichlarga zararli hayvonlar hisobida qaralar edi. Buning natijasida qator yirtqich hayvonlar shavqatsiz qirib tashlanib, tabiatga katta zarar etkazildi. Chunki, ular o'lja bo'lgan kasal zaif individlarni populyatsiya tarkibidan tozalab katta foyda keltiradi va sanitar vazifani bajaradi. Jumladan, hatto bo'rilarni shimol bug'ulari podalarining tez ko'payishi va yashovchanligining ortishiga yordam berishi aniqlangan. 
Yirtqichlar tabiiy tanlanishning borishini tezlashtiruvchi omillardan biridir.

Yirtqichlardan farqliroq parazitizm turning xo'jayinligi tor ma'nodagi maxsus moslashganligidir. Masalan, odam askaridasi faqat odam ingichka ichagida yashasa, ot askaridasi esa faqat otlardagina parazitlik qiladi. Chunki parazitlar o'z xo'jayinidan faqat ozuqa manbai sifatidagina emas, balki undan vaqtincha yoki doimiy yashash muhiti sifatida ham foydalanadi.

Parazitlar xo'jayin tanasida xavf-xatardan to'liq himoyalangan parazitlik hayotiga moslashuv tufayli, ularda qator organlar reduktsiyalangan, morfofiziologik soddalashishga sabab bo'lgan. Masalan, tasmasimon chuvalchanglarda ovqat hazm qilish sistemasi butunlay qisqarib ketgan, nerv sistemasi ham reduktsiyaga uchragan. Shiralar o'simliklarning bargi, novdasi va ildiz qismida parazitlik qilishi bilan birga, ular faoliyati natijasida ozuqa o'simligining turli qismlarida hosil bo'lgan turli-xil gallar ichida ham hayot kechirishga moslashgan. Bunday gallarning bahorda teraklar, qayrag'och bargi va novdalarida ko'plab uchratish mumkin. Gallar ichidagi shiralar ancha sodda tuzilgan, mo'ylablari 4 bo'g'imli, ko'zlari 3 fasetkali, kam harakatli bo'ladi. Ularning hayoti uchun muhim sanalgan dum va shira naychalari bo'lmasligi mumkin.

Tabiatda parazit-xo'jayn munosabati stabillashmagan taqdirda parazitlar faoliyati juda xavfli bo'ladi. Tripanasomalar, antilopalar qonida yashaganda, bu hayvonlarga umuman zarar keltirmaydi, lekin ular antilopalardan tse-tse pashshalari orqali odamlarga o'tgach, ya'ni transmissiya sodir bo'lgach o'ta xavfli, o'lim bilan tugallanuvchi uyqu kasalligini yuzaga chiqaradi.

Shuningdek, tasodifan kelib qolgan zararkunandalar mahalliylariga nisbatan qishloq xo'jaligi ekinlariga kuchli zarar keltiradi. Bu o'rinda kolorado qo'ng'izi tipik misol bo'la oladi. O’z vatani - Shimoliy Amerikada yovvoyi ituzumdoshlar bilan oziqlanadigan bu hasharot asrimiz boshlarida tasodifan kartoshka bilan G'arbiy Evropaga olib kelinishi, ularning bu joylarda ko'payishi, tez tarqalishi va kartoshkazorlarga jiddiy zarar keltirishiga sabab bo'ldi. Respublikamizning kartoshkachilik xo'jaliklarida ham bu zararkunanda ko'plab uchrab turadi. Keyingi yillarda Respublikaning qator tumanlarida (Farg'ona, Namangan va b.) kartoshka dalalarida uning zarari sezilarli bo'lmoqda. 
Yirtqich-o'lja, parazit-xo'jayin munosabati tabiatda moddalar almashinuvida va turlar sonining boshqarilishida muhim rol o'ynaydi. Paxta dalalari va bog'larda zararkunanda hasharotlar sonini kamaytirib, boshqarib turuvchi asosiy omillardan biri yirtqich va parazit organizmlarning faoliyatidan boshqalari qulaygina foydalanishi mumkin. $\mathrm{Bu}$ organizmlar o'rtasida kommensalizm - bir tomonlama foydali munosabat bo'lib, ikkinchi organizmga umuman zarar etmaydi. Yirik yirtqichlarning o'lja qoldiqlari bilan o'limtikxo'r qushlarning ovqatlanishi bunga misol bo'ladi. Keyinchalik chumchuqsimonlar o'txo'r hayvonlar chiqindilaridan to'liq hazm bo'lmagan donlarni topib eydi.

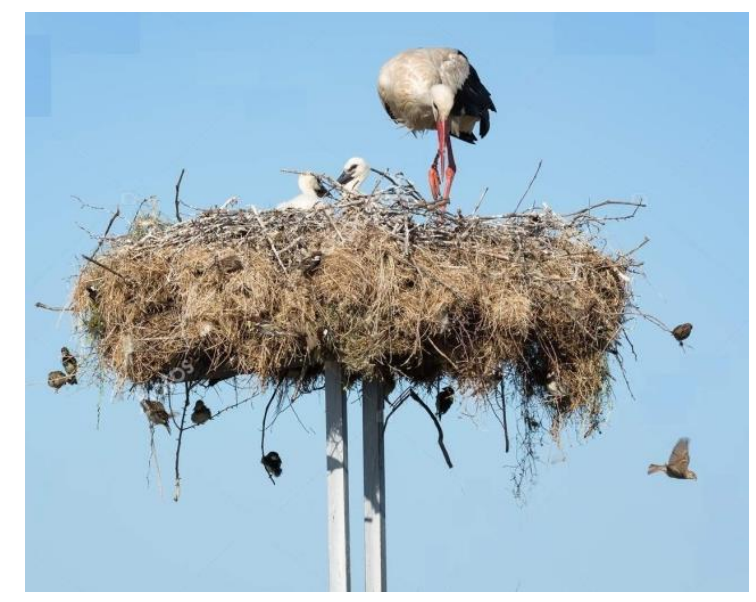

10-rasm. Laylaklardagi uya kommensalizmi

Tabiatda uya kommensalizmi ko'proq kuzatiladi. Laylaklarning ko'p yillik uyalari shox-shabbalari oralarida chumchuqlar ham bola ochadi, sut emizuvchilar uyalarida - oqqanotlilar, kanalar, bitlar, burgalar kabi ko'plab bo'g'imoyoqlilar yashaydi. Kommensalizm turlarning yanada yaqinroq bog'lanishiga imkon beruvchi biotik munosabatdir.

Organizmlar o'rtasidagi o'zaro munosabat - mutualizm tabiatda keng tarqalgan. Bu aloqa vaqtli yoki doimiy bo'lishi mumkin. Doimiy foydali o'zaro munosabatni simbioz deb yuritiladi.

Инсонлар хаётида бир қанча жониворлар уларга доимо хамрох бўлиб, уларнинг хизматини қилиб келади. Эхтимол, миллион-миллион йилар яшаган одамлардан бири ўрмондан қандайдир бўри боласини топиб олиб уни капасида ўзидан ошган овқатлик билан боқиб, тарбиялаб катта қилган. Кейинчалик бу бўри боласи эгасига жуда 
мехрибон бўлиб уни қўриқлаш, хайвонларни хам турли йиртқичлардан химоя қилиш, капа қўриқлаш каби хизматларни қила бошлаган. Шундай қилиб минглаб йиллар оша инсон ўзга бўрилардан хосил бўлган энг яқин дўсти ит билан бирга яшашга ўтган. Ит одамга бошқа турли ёввойи хайвонларни хам хонакилаштиришга ёрдам берган.

Одам ит мисолида ўзга дўстлар топиб олганидек, табиатда жуда кўп хайвон ва ўсимликлар хам ўзларига мос бўлган шундай ёрдамчиларни топиб олган. Аниқланишича, одам пайдо бўлмасдан анча олдинги даврларда турли хайвонлар ўзларига шундай ёрдамчиларни топиб, бирга яшаб келар экан. Агар кишилар 40 дан ортиқ ёввойи хайвонларни аста-секин маданийлаштириб ўзларига ёрдамчи қилиб олган бўлсалар, биргина чумолиларнинг ўзи бирга яшаш учун одамларга нисбатан 50 баробар кўп ёввойи хайвонларни "хонакилаштириб" улардан ўз хаётлари мобайнида фойдаланишга ўтишган. Бу митти жониворлар уяларида минглаб чумолилардан ташкил топган жамоа бўлиб яшашларига қарамай яна уйларидан турли хайвонларга жой бериши, улар билан хамкорликда яшашга ўтишлари хайратлидир.

Биз турли ахборот манбаларидан мушукни жўжаларни асраб олганлиги, она товуқ эса мушук болаларини етаклаб, химоя қилиб юриши, тулкининг хўроз билан дўстлиги кабиларни биламиз. Бундай хабарлар газета, журналларда сенсаtsiя тарзида берилади. Бироқ, табиатда бундай ходисалар жуда кўп учрайди. Чўлларда, ўтлоқларда юрганимизда эгнимизга қўйтиканнинг игнали мевалари ёпишиб қолиши кўпчиликка маълум. Айниқса чорва моллари далаларда ўтлаб юрган пайтларида уларнинг юнгларига бундай мевалар жуда кўплаб ёпишиб қолади. Ундан ташқари, қўйтикан билан озиқланган жониворлар ичагига тушган ўсимлик мевалари хазм бўлмай, маълум вақтлар ўтганидан сўнг хайвон қаерга борган бўлса ўша ерда тупроққа бу мева ахлат билан тушиб қолади. Демак хайвонлар бу ўсимликни табиатда кенг тарқалишига хизмат қилишар экан.

Қизиғи шундаки, янтоқ уруғи хайвон ичагига тушмаса ундан янги ўсимлик ривожланмас экан.

Инсонлар жуда кўп нарсаларни, билимларни табиатдан олишади. Кишилар ўсимлик ва хайвонларни тузилишин ўрганиш билан жуда бой илмий маълумотларни тўплаган бўлсалар, эндиликда бу организмларни ўзаро биргалашиб тотувликда яшаш сирларни хам ўрганиб бормоқда.

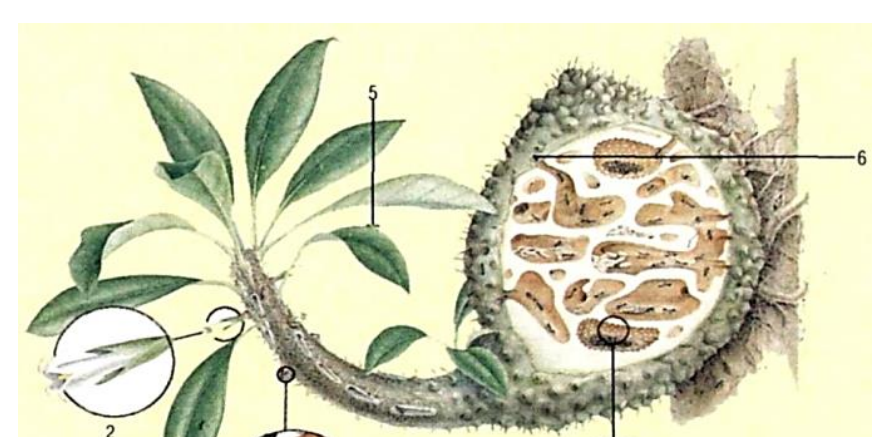


1. Чумоли ўсимликнинг нектар чиқарувчи безидан нектарни ўзлаштирмоқда, 2. Гул асосининг ривожланиши, 3,4-ўсимликлар чумолиларнинг чиқиндисини сўнгалсимон ўсимталари орқали ички юза қатламга сўриб олади, 5. Чумолиларнинг бўшлиқлар орқали ўтиши натижасида ўсимлик танаси ва юзаси йириклашади, 6. Бу бўшлиқлар бир-бири билан туташмайди, балки ташқарига алохида очилади.

\section{Расм-1. Ўсимлик ва чумоли орасидаги симбиоз}

Irisomyrmex авлодига мансуб чумолилар билан Myrmecodia авлодига мансуб уссимликлар орасидаги симбиоз хам ахамиятга сазовор ходисалардан биридир. Бу ўсимликлар турли дарахтлар танасида яшовчи, бу жойларнинг тупроқлари минерал моддаларга камбағал жойларда яшаганликлари туфайли доимо минерал моддаларга талабчан бўлишади. Бу моддаларга бўлган талабларни ўсимлик чумолилар билан хамкорликда қондиради. Бу симбиоздан чумолилар хам ўсимлик ўзаро фойда кўришади. Гап шундаки, чумолилар бу ўсимлик гулининг асосида жойлашган (2) нектарларида хосил бўладиган шира билан озиқланишади (расмдаги 1-унсур). Чумолилар гулда ўзларидан чиқарган ташландиқлари (3) эса гул учун яхши туйимли моддалардан саналади. Гул чумоли ташландиқларини ўзининг аррасимон шаклдаги таглиги билан (4) сўриб олади.

Ko'p hollarda “zohid qisqichbaqa"lar o'z chig'anog'i ustiga 1-2 ta aktiniyani "mingashtirib" oladi, bunda aktiniyaga "transport vositasi" bo'lib xizmat qilgan qisqichbaqa xavf-xatardan holi bo'ladi, aktiniya ushlagan oziqning bir qismi bilan yaxshigina ovqatlana oladi. Chumolilar ham shiralar bilan simbioz yashaydi. Chumolilar shiralar naychalarini mo'ylabi bilan "qitiqlaganda" hasharot shirin suyuqlik tomchisini chiqaradi, bu tomchi hisobiga chumoli ovqatlanadi. Bunday paytda shiralar har minutda tomchitomchi shirin suyuqlik ajratib turadi. Shuning uchun ham agar shiralar biror o'simlikda ko'payib ketsa, chumolilar ularni daraxtning boshqa shoxiga yoki boshqa o'simlikka ko'chirib o'tkazadi. Hushyorlik bilan ularni tugmacha 
qo'ng'iz, oltinko'z, qandalalar va boshqa dushmanlardan himoya qiladi. Shuning uchun ham tabiatda ko'pchilik shiralar doim chumolilar bilan birga yashaydi. O'simliklardan lishayniklar zamburug'lar va suv o'tlarining birgalikda - simbioz yashashi natijasida shakllangan organizmlardir.

Turlarning ekologik talab darajasi qanchalik yaqin bo'lsa, tabiatda qarama-qarshi munosabat - raqobat vujudga keladi. Bunday turlar birga yashagan paytda ular noqulay sharoitga tushib qoladi. Ya'ni ovqat manbalari, yashash joyi va boshqalar chegaralanib qoladi. Raqobat o'zaro munosabatda bo'ladigan ikkala organizm uchun ham salbiy ta'sir etuvchi biotik aloqadir. Shuning uchun ham bir xil ekologik talab darajasidagi turlar bir joyda yashasa, ertami, kechmi biri ikkinchisini siqib chiqaradi. Raqobat bir tur ichida ham turlararo munosabatda ham uchraydi.

Qarama-qarshi munosabatlar tur ichida, turlararo to'g'ridan-to'g'ri yoki nisbiy bo'lishi mumkin. Turlararo to'g'ridan-to'g'ri raqobat yirtqich-o'lja, parazit-ho'jayin munosabatida ifodalanadi. Turlararo nisbiy kurashda o'z ozuqasini osonlik bilan topa oladigan hayvon ikkinchisiga nisbatan ustun bo'ladi. O'txo'r sut emizuvchilar va chigirtkalar ikkalasi ham fitofag bo'lgani uchun ular o'rtasida nisbiy qarama-qarshilik kuzatilmaydi. Tur ichidagi to'g'ridan-to'g'ri raqobat yashash joyi ikkinchisi uchun ovqat bo'ladi. G'o'za tunlami kapalagi odatda tuxumlar soni bitta tupda 3-5 tagacha borishi mumkin, bunda eng avval tuxumdan chiqqan ko'sak qurti o'zidan keyingi rivojlangan "nasllarini" eb tugatadi va yakka o'zi mo'l ozuqa hisobiga to'liq voyaga etadi. Tur ichidagi nisbiy raqobat kuchsiz individlarning ko'plab qirilishiga sabab bo'ladi. Jumladan, bo'ridan ikki quyon qochayotgan bo'lsa, tur ichidagi nisbiy kurashda albatta tez yuguradigan epchil va aqliroq quyon yutib chiqadi.

Biotik munosabatlar ichida neytralizm ma'lum hududda birga yashayotgan ikki turni bir-biriga ijobiy ham salbiy ham ta'sir ko'rsatmasligida ifodalanadi. O'rmondagi olmaxon va loslar umuman raqobatda bo'lmaydi. Lekin o'rmondagi yong'in, zararkunandalarning ko'payishi bularga turli darajada ta'sir etadi.

Evolyutsiya jarayonida o'simlik va hayvonlarning bir-biri bilan yaqin aloqasi birgalikdagi taraqqiyoti ularda o'zaro moslanishlarning vujudga kelishiga sabab bo'lgan. Shuning asosida o'simliklarning hasharotlar 
yordamida changlanishi, changlatuvchi hasharotlar, qushlar, sut emizuvchilar guruhlari vujudga kelgan: ko'pchilik o'simliklar urug'i hayvonlar yordamida tarqalishga moslashgan. O'simlik va hayvonlar o'rtasidagi biotik muonsabatlarni eng muhimi ularning ovqat orqali bo'ladigan trofik aloqasidir. O'simliklar hayvonlar uchun ovqat manbai bo'lib xizmat qiladi. O'simlikxo'r hayvonlarning ozuqaga nisbatan maxsus moslanishi xilma-xil bo'lib, ularning ichida so'ruvchi fitofaglar (shiralar, qandalalar, tsikadalar) alohida o'rin tutadi. Ko'pchiligi esa o'simliklarning vegetativ yoki er osti organlari hisobiga yashaydi. Ko'sak qurtlari g'o'zaning o'sish nuqtasi gul va hosil elementlarini zararlasa, tomir qurtlari esa erta bahorda ekin tomirini qirqib zarar etkazadi.

O'simliklarning changlanishida hayvonot dunyosining roli katta. Tabiatda 1600 ga yaqin qushlar bu muhim biologik jarayonni amalga oshirishda qatnashadi. Janubiy Amerika va markaziy Amerika o'simliklari ma'lum qismining changlanishida ko'rshapalaklar alohida o'rin tutadi. Bizning sharoitimizda o'simliklarning, ayniqsa g'o'za gulining changlanishida asalarilar muhim ahamiyatga ega. Aniqlanishicha, ishchi asalari 1 minutda 12 ta, 1 kunda esa 4000 ta gulga qo'nadi. Bir asalari oilasida 10000 dan 50000 ga qadar individlar bo'lishi nazarda tutilsa, ular bir kunda $360 \mathrm{mln}$. ga yaqin gulni changlata oladi.

Hayvonlar o'simliklarning tarqalishida ham katta ahamiyatga ega. O'simlik urug'lari va sporalarining hayvonlar yordamida tarqalishi zooxoriya deb ataladi. Hayvonlar urug'lardan ozuqa zahirasini yig'adi. Ularni bir joydan ikkinchi joyga siljitadi, bunda urug'larning bir qismi qulay sharoitlarga tushib, unib chiqadi. Sariq chumoli koloniyasi a'zolari uyadan 70 m. radiusda 36000 tagacha o'simlik urug'ini sochar ekan. Tuyoqli hayvonlarning yunglariga maxsus moslamalar yordamida ilashib qolgan urug'lar ba'zan minglab km, gacha tarqala oladi. Bulardan tashqari, ko'pchilik o'simliklarning urug'lari hayvonlar xazm sistemasida butunligicha saqlanib qoladi va ularning ekskrementlari bilan boshqa joylarga tushib unib chiqadi. Maynalarning chiqindilaridan gilos, olcha, uzum, itburun, shilvi va boshqa mevalar urug'larini topish mumkin. Otlar, qo'ylar va yirik shoxli qoramollar go'ngida yovvoyi boshoqlilar urug'lari ko'plab saqlanib qoladi. Hisoblanishicha, 1 gektarga solingan 60 tonna ot go'ngida 90 mingtagacha yovvoyi o'simliklar urug'i bo'ladi. 
Yuqorida ko'rib o'tilgan biotik munosabatlar turlararo va tur ichidagi bo'ladigan murakkab aloqalarning eng xarakterlilaridir. Bu munosabatlarning barchasi tiriklikning yagona birlik - biotsenoz darajasida bir-biriga bog'lab turadi. Shuning uchun ham biotsenozdagi organizmlar uning ajralmas tarkibiy qismi bo'lib, ularni foydali yoki zararli turlarga shartli ajratiladi, chunki tabiatdagi hayvonot dunyosi barcha vakillarning o'z o'rni bor.

\section{Biologik maromlar}

Tirik tabiatning muhim xususiyatlaridan biri unda sodir bo'lib turuvchi hayotiy jarayonlarning uzluksizligi va davriyligidir. Barcha tirik organizmlar hayoti, hujayradan tortib biosferaga qadar muayyan maromga asoslangan har bir tur kun va yil davomida, shuningdek, mavsumiy hayotini boshqaradi. Biologik marom (ritm) - organizmlardagi hayotiy jarayonlarni vaqt birligi davomida ma'lum izchillikda, qat'iy ravishda boshqarilishidir.

Er yuzidagi barcha organizmlarda ichki va tashqi maromlar kuzatiladi.

Tashqi maromlar geofizik tabiatga ega, ular erning o'z o'qi va quyosh atrofida aylanishiga nisbiy bog'langan. Bu jarayonga bog'liq holda mavsumiy va sutkalik o'zgarishlar ro'y beradi, harorat, namlik, yorug'lik kuchi, bosim va boshqa omillar o'zgaradi. Quyoshning faolligi ham tabiatga o'z ta'sirini o'tkazadi. Jumladan, quyosh uchun 11 yillik davriylik xos. Tashqi o'zgarishlarning barchasi tirik organizmlarning hayotiy jarayonlarga va fe'latvorlariga ta'sir etadi.

Ichki davriylik, birinchi navbatda, organizmlardagi fiziologik maromlardir. Davriylik va maromlar RNK va DNK sintezida, oqsillarning sintezlanishi va yig'ilishida, mitoxondriy faoliyatida, hujayra bo'linishida, muskullar qisqarishida, nafas olishda, yurak faoliyatida va boshqa qator hayotiy jarayonlarda kuzatiladi. Barcha ichki maromlar va davriylik uyg'unlashib organizmlarning fe'l-atvorlarini belgilaydi. Ichki va tashqi maromlar o'rtasidagi ketma-ketlik va mutanosiblik vaqt davomida ifodalanadi. Shuning uchun ham organizmlar uchun vaqt eng asosiy omil sifatida ekologik ahamiyatga ega. Barcha organizmlar o'zlaridagi tashqi va ichki biomaromlar nuqtai nazaridan vaqtga doimo moslashib borishlari lozim. Organizmlardagi ko'pchilik hayotiy jarayonlar, faollik va boshqa holatlar, 
asosan, geofizik tsikllariga mos keladi. Shuning uchun ham bu maromlarning barchasini adaptiv sutkalik maromlar deb nomlanadi. Organizmlardagi adaptiv biologik maromlar fiziologik moslanish sifatida tashqi muhitning ekologik o'zgarishlariga ularning javob reaktsiyasidir. Shu jihatdan ular organizmlarda kechayotgan sof fiziologik maromlardan (nafas olish, qon aylanish va boshqalar) farqlanadi.

Bir hujayralilardan odamlarga qadar barcha organizmlarda sutkalik marom kuzatiladi. Insonlarda 100 dan ortiq sutkalik o'zgarishlar bilan bog'langan fiziologik jarayonlar aniqlangan. Jumladan, uyqu va uyg'oqlik, tana haroratining o'zgarishi, yurak urishi maromi, nafas olish maromi, siydik tarkibi va hajmi, ter ajralishi, jismoniy va aqliy faollik va boshqalar. Aniqlanishicha, soxta oyoqlilarning ko'payish tezligi ham kun davomida o'zgarib turar ekan.

Hayvonlardagi uyqu (tinim) va uyg'oqlik (tiyraklik) davrning mutanosibligiga mos ravishda, tungi va kunduzgi faol hayvonlar guruhlari farqlanadi. Kunduzgi faol hayot kechirish tovuqsimonlarda, qator chumchuqsimonlarda, ayrim kemiruvchilarda, chumolilarda va ninachilarda kuzatiladi. Tungi faol hayvonlar guruhiga tipratikan, ko'rshapalak, boyo'g'li, yovvoyi cho'chqalar, mushuksimonlar, suvaraklar va boshqalar mansub. Ayrim hayvonlar esa tunda va kunduzi ham bir xil maromda faollikda bo'ladi yoki bardamlik va nisbiy tinim davri ularda bir me'yorda qaytariladi. Bunday maromlar ko'p fazali - polifaz maromlar deb nomlanadi (masalan, erqazarlar, ayrim yirtqichlar va boshqalar).

Ko'pchilik hayvonlardagi sutkalik maromlar ulardagi harakat faolliklarining o'zgarishlariga mos keladi, fiziologik jarayonlarda esa ortiqcha o'zgarishlar ro'y bermasligi mumkin. Bu holat kemiruvchilarda aniqlangan. Sutkalik o'zgarishlarning fiziologik jarayonlarga bo'lgan sezilarli ta'siri ko'rshapalaklarda yaxshi ifodalangan. Ular yozning kunduz kunlarida tinim holatida bo'lib, poykilotermlik xususiyatini namoyon etadi. Ularning tana harorati atrof muhitnikiga barobar bo'lib, nafas olishi, yurak urishi, sezgirligi keskin pasayadi. Oqshom va tunda gomoyoterm bo'lgan bu hayvonlarning muskullari jadallik bilan qisqaradi va kimyoviy termoregulyatsiya hisobiga ajralgan issiqlik tana haroratini ko'taradi. Natijada ulardagi harakatlar aniq va tez bo'ladi. 
Ayrim turlardagi faollik sutkaning muayyan bir vaqtiga mos keladi, boshqalarida esa sharoitga qarab u yoki bu tomon siljishi mumkin. Masalan, qisqichbaqasimonlardan zahkashlar va ayrim qo'ng'izlar faolligi bevosita tashqaridagi harorat va namlikka bog'liq holda o'zgarishi mumkin. Ular uyalaridan barvaqt ertalab va kechqurun (ikki fazali tsikl) yoki faqat tunda (bir fazali tsikl) yoki kun bo'yi chiqishi mumkin.

Endogen maromlardan farqini tajriba asosida ham izohlash mumkin. Masalan, ko'pchilik hayvonlar doimiy harorat, namlik va yorug'lik sharoitida saqlangan taqdirda ham ulardagi sutkalik maromlar buzilmagan va aniq izchillikda uzoq muddat davomida qaytarilganligi kuzatilgan. Bunday barqaror ichki maromlar tsirkad (circa - atrofida, dies - kun, sutka) maromlar nomini olgan. Ko'rshapalaklar tunda falaj bo'lganlari uchun ma'lum bir vaqtdagina uyg'onadilar. Lekin ular kun bo'yi to'liq qorong'ulikda saqlangan taqdirda ham sutkalik maromini bemalol qaytaradi. Bunda istisno tarqasida ko'rshapalaklarning ba'zilari boshqalaridan faollikni bir oz oldin boshlagan bo'lsa, qolganlaridagi faollik bir necha daqiqadan so'ng boshlangan.

Insonlardagi tsirkad maromlar turli vaziyatlarda: g'orlarda, germetik kameralarda, suv osti kemalarida o'rganilganda shu narsa ma'lum bo'ldiki, ulardagi sutkalik maromlardan cheklanishlar har bir insonning asab tizimi va tipologiyasiga bog'liq ekan. Shu jihatdan bir oila a'zolarining tsirkad maromlari turlicha bo'lishi tabiiy.

Tsirkad maromlar mos bo'lgan fe'l-atvor reaktsiyalaridagi stereotip organizmlarni sutkalik o'zgarishlariga oson moslasha olish imkoniyatini beradi. Lekin hayvonlarni bir joydan ikkinchi joyga siljishida, tarqalishida nisbatan barqaror fe'l-atvor reaktsiyalari bir muncha noqulaylik tug'dirishi tabiiy. Bu holat barcha hayvonlarni keng areallarda erkin tarqala olish imkoniyatini chegaralab qo'yadi. Masalan, kulrang kalamushlar keng ekologik valentlikka ega va ulardagi tsirkad maromlar sutkalik o'zgarishlarga oson moslashadi. Qora kalamushlardagi bunday imkoniyatlar bir muncha chegaralangan. Shuning uchun ham kulrang kalamushlar butun er yuzi bo'ylab deyarli keng tarqalgan. Organizmlardagi tsirkad maromlar ma'lum chegarada o'zgarishi ham mumkin. Masalan, odamlar o'zi yashayotgan joydan uzoqqa, boshqa mintaqaga borib qolganda ulardagi tsirkad maromlar "eskicha" namoyon bo'ladi, fiziologik jarayonlar izchilligida buzilish ro'y beradi. Oradan 
bir necha kun yoki haftalar o'tishi bilan yangi joyning sutkalik o'zgarishlariga organizm moslashib qoladi. Tsirkad va kunlik maromlar asosida organizmlar aniq vaqtni belgilay oladi va ulardagi bu xususiyat "biologik soat" nomini olgan. Yuqori darajada takomillashgan hayvonlardagi murakkab tug'ma hattiharakatlarning namoyon bo'lishida vaqt muhim ahamiyatga ega, ya'ni ular vaqtni mo'ljalga oladi, jumladan, qushlarning safar uchishlarini boshlanishi va tugallanishi, hayvonlarning mavsumiy migratsiyalari va boshqalar.

Dengiz va okeanlarda suvning ko'tarilishi va pasayishi sutka davomida ikki marta hamda oyning boshi va oxirida kuzatiladi. Shuning uchun qirg'oq mintaqalarida yashayotgan hayvonlarga 24 soat davomida yorug'lik va boshqa omillarning o'zgarishidan tashqari suvning ko'tarilishi va pasayishi ham ta'sir etadi. Ushbu maromlarga qirg'oqlarda yashovchi organizmlar moslashgan. Jumladan, ustritsalar (mollyuskalar) suv to'lqinining qaytishi vaqtida pallalarini yopib olib butunlay ovqatlanmaydi. Kaliforniya sohillarida tarqalgan aterina baliqlari rivojlanish davrida oy davomida suvning eng yuqori ko'tarilib tushishidan foydalanadi. To'lqin suvining ko'tarilishi kuzatilgan vaqtda urg'ochisi qirg'oqdagi qum ostiga ivildirig'larini tashlaydi, oradan 15 kun o'tgandan so'ng ulardan chavaqlar chiqadi va suvning ikkinchi marta eng yuqori ko'tarilib tushishidan suvga tushib ketadi.

Oylik maromlar quruqlik va dengizda yashovchi ayrim hayvonlarda kuzatiladi. Hayvonlarning yorug'likka javob reaktsiyasi kuchsiz magnit maydoni ta'siri yoki mo'ljal olish tezligi kabilar oylikmarom bilan bog'liq. Oylik maromlar palolo ko'p tukli chuvalchangining ko'payishida, xironomid chuvalchanglari va kunliklarning g'uj bo'lib yig'ilishida kuzatiladi.

Yillik maromlar. Fizik omillarning yil va mavsum davomidagi o'zgarishi hayvonlardagi xilma-xil moslanishlarni vujudga keltirgan. Bu holat ularning mavsumiy faolligi, ko'payishi, migratsiyasi, noqulay mavsumlarga moslanishlarida o'z ifodasini topgan. Bahorgi uyg'onish, yozgi faollik va tinim, qishki yillik maromlarga asoslangan. Hayvonlardagi yillik maromlar asosan endogen bo'lib uni tsirkan (appiya - yil) maromlar deb nomlanadi. Kun uzunligining o'zgarishi hayvonlardagi yillik maromlarni boshqarilishida asosiy ahamiyatga ega.

\section{Hayvonlarning hayotiy shakllari}


Hayvonlarning yashash muhitiga moslanish usullaridan biri morfologik adaptatsiyadir. Hayvonlarning tashqi muhit omillariga morfologik moslanish bilan namoyon etadigan yashash tarzi hayotiy shakllar deyiladi. Hayvonlarning tarixiy rivojlanishi jarayonida yashab turgan muhitga mos ravishda morfologik va ekologik moslanish xususiyatlari shakllangan. Rus ekologi D.N.Kashkarov birinchilardan bo'lib zooekologiyada hayotiy shakllarni ilk marta tasnifladi. Olimning ta'kidlashicha, hayvonlarning hayotiy shakllarini tasniflashda tashqi muhitning umumiy xususiyatlaridan tashqari, ayni shu sharoitdagi harakatlanish imkoniyatlari, ozuqaning harakteri va boshqalar inobatga olinishi lozim. D.N.Kashkarovning tasnifiga ko'ra, hayvonlarning hayotiy shakllari quyida berilgan.

\section{Hayvonlarning hayotiy shakllari tasnifi}

\section{Hayvonlarning iqlimga bo'lgan munosabati bo'yicha:}

\section{Covuq qonli hayvonlar}

Yil davomida faol hayvonlar

Yilning ma'lum davrida faol hayvonlar

A) yozgi uyquga ketadiganlar

B) qishki uyquga ketadiganlar

\section{Issiqqonli hayvonlar}

A. O'troq hayot kechiruvchilar

1. Yil davomida faol hayvonlar

2. Yilning ma'lum davrida faol hayvonlar

a) yozgi uyquga ketadiganlar

b) qishki uyquga ketadiganlar

B. Mavsumiy hayot kechiruvchilar

1. Uya quruvchilar

2. Qishki faol hayvonlar

3. Yozgi faol hayvonlar

4. Ko'chib yuruvchilar 
Hayvonlarning harakatlanishiga moslanish bo'yicha:

\section{Suzuvchi hayvonlar}
A) nektonlar
B) planktonlar
V) bentoslar

\section{Suvda va quruqlikda yashovchilar}
A) sho'ng'ib yuruvchilar
B) sho'ng'imaydiganlar va boshqalar
V) faqat suvdan ovqat topadiganlar

\section{Kavlovchi hayvonlar}

Butunlay er kavlovchi (hayoti to'lig'icha tuproq ostida o'tadigan) hayvonlar

Nisbatan er kovlaydiganlar (ma'lum muddatga tuproq yuzasiga chiqadigan) hayvonlar

\section{Quruqlikda yashovchi hayvonlar}

1. Uya qurmaydiganlar
A) yuguruvchilar
B) sakrovchilar
V) o'rmalovchilar (sudraluvchilar)

2. Uya quradiganlar
A) yuguruvchilar
B) sakrovchilar
V) o'rmalovchilar (sudraluvchilar)

3. Qoyalarda hayot kechiruvchilar

\section{Daraxtlarga o'rmalab chiqib yashovchilar}
A) daraxtlardan tushmasdan hayot kechiruvchilar
B) daraxtga tirmashib chiquvchilar

\section{Havo muhitida yashovchilar}

A) havoda oziq topuvchilar 
B) ozuqasini havoda turib ta'qib etuvchilar

Hayvonlarning namlikka bo'lgan munosabati bo'yicha:

1. Namlikni oluvchilar - gigrofillar

2. Quruqlikni sezuvchilar - kserofillar

Hayvonlarning ozuqa xarakteri bo'yicha:

1. O'simlikxo'rlar
A) ko'katxo'rlar
B) donxo'rlar
V) mevaxo'rlar

2. Hammaxo'rlar

3. Yirtqichlar
A) hasharotxo'rlar
B) etxo'rlar

4. O'limtik va o'laksaxo'rlar

Hayvonlarning ko'payish joylari bo'yicha:

1. Er ostida ko'payuvchilar

2. Er yuzasida ko'payuvchilar

3. O't-o'lanli yaruslarda ko'payuvchilar

4. Butalarda ko'payuvchilar

5. Daraxtlarda ko'payuvchilar

6. Turli bo'shliq va uyalarda ko'payuvchilar

Hayvonot dunyosining hayotiy shakllarini umumiy tarzda ifodalovchi ushbu ekologik tasnif, hozirgi paytda keng talqin etilgan va har bir tasnifiy guruhlar doirasidagi xususiy hayotiy shakllar ham aniqlangan. 


\section{3-BO'LIM. POPULYATSIYALAR EKOLOGIYASI}

Populyatsiya - muayyan joyda uzoq muddat yashagan bir turga mansub individlar yig'indisi. Populyatsiyaning chegarasi odatda tarkibida ayni tur bo'lgan biotsenoz chegarasiga mos keladi. Populyatsiya turning eng quyi tuzilma birligidir. To'da, gala, prayd va partsellyar guruhlar birlik bo'la olmaydi, chunki ular bir mavsumda tarqalib ketadi. Uch xil: geografik, ekologik va mahalliy populyatsiyalar farqlanadi. Muayyan tur tarqalgan arealdagi muhit omillarining xilma-xilligi populyatsiyadagi turli moslanish belgilarining shakllanishiga sabab bo'ladi.

Populyatsiyadagi genetik birlik turning biologik aloqalanishiga va barqororligini saqlanishiga yordam beradi.

Populyatsiyadagi individlararo munosabatlar turlararo shakllangan biotik munosabatlarga bir muncha yaqin, ayniqsa, ular o'rtasidagi mutualistik va qarama-qarshi munosabatlar ko'proq kuzatiladi. Populyatsiyadagi individlarning bir-biriga bo'lgan ta'siri muhitning abiotik omillari ta'siriga nisbatan ham yuqori bo'ladi. Populyatsiyagagina xos bo'lgan munosabatlar bu bog'lanishlardir. Populyatsiyadagi individlar o'zaro chatishib erkin nasl beradi. Partenogenetik ko'payish xususiyatiga ega bo'lgan hayvonlarning populyatsiyalari o'ziga xos toza chiziqlarni hosil qiladi. Populyatsiyadagi individlar chegaralangan ozuqa resurslaridan unumli foydalanadi va erkin nasl qoldiradi.

Populyatsiyaning muhim xususiyatlaridan biri o'zining son jihatdan idora etishi - miqdor zichligini boshqarishidir. Muayyan sharoitda optimal miqdor zichligini saqlab turilishi populyatsiya gomeostazi deyiladi. Barcha populyatsiyalar uchun quyidagi xususiyatlar xos:

-populyatsiyadagi individlar soni (mo'lligi);

-zichligi;

-tug'ilish;

-o'lim (nobud bo'lish);

-populyatsiyadagi o'sish;

-o'sish sur'ati.

Populyatsiyalar uchun shakllanish tuzilmalari ham xos bo'ladi. 
Individlarning ma'lum hududda tarqalishi, jins va yosh nisbatlari, morfologik, fiziologik, fe'l-atvor va genetik xususiyatlari populyatsiyaning shakllanish tuzilmasini ifodalaydi. Har bir turning populyatsiyalari va o'ziga xoslik uning biologik xususiyatlari va tashqi muhit omillariga bog'liq bo'ladi.

\section{Turning populyatsion tuzilmasi}

Tur muayyan hududda tarqalib, bir yoki bir necha populyatsiyalarni hosil qilishi mumkin. Turning tarqalish hududi arealni belgilaydi. Turning populyatsion tuzilmasi uning biologik xususiyatlari, harakatlanishi, tabiiy to'siqlarini enga olish imkoniyatini, shuningdek, arealdagi geografik va muhit omillarining xilma-xilligiga bog'liq. Tur keng hududda tarqalib turli mintaqalar bo'ylab doimiy harakatda bo'lsa, uning populyatsiyalari soni ham yuqori bo'ladi. Shimol bug'ulari va oq tulkilar keng mintaqalarda mavsumiy migratsiya qiluvchi hayvonlardan sanaladi. Oq tulkilar asosiy ko'payish joylaridan bir necha yuz, ba'zan esa minglab kilometr uzoqlashishi mumkin. Bu holat shimol bug'ulari va oq tulkilarga monand turlarning populyatsiyalari o'rtasidagi chegaralar odatda yirik geografik to'siqlar - daryolar, jarliklar, tog'lar orqali o'tadi. Ba'zida, tur jadal harakatlanish xususiyatiga ega bo'lsa, nisbatan katta bo'lmagan arealda yagona populyatsiyani hosil qilishi mumkin. Masalan, echkilar urug'iga kiruvchi Severtsov turi katta Kavkaz tog'ining g'arbida, Dog'iston turi esa shu tog'ning sharqiy qismida, har biri alohida populyatsiyalarni hosil qiladi. Ular 4 ming metr balandlikkacha ko'tariladi. Qishda sovuq va qordan qochib, tog'ning pastrog'iga tushadi.

Harakatlanish ancha sust bo'lgan hayvonlarning yashash joylariga bog'liqlik darajasi yuqori bo'ladi, ular bir necha sondagi mayda populyatsiyalarni hosil qiladi. $\mathrm{Bu}$ holat tarqalgan hududdagi landshaftlar xilma-xilligini ham ifodalaydi. Tog'li mintaqalarda bunday turlarning hududiy differentsiallashishi ularning fe'l-atvorlariga bog'liq holda past tekisliklardagiga nisbatan ancha murakkab bo'ladi. Masalan, qo'ng'ir ayiqlar yashash joylariga o'ta bog'langan, shuning uchun ham bir tog'ning o'zida mayda guruhlar shaklida yashaydi. Qo'ng'ir ayiqning har bir guruhi o'ziga xos xususiyatlari bilan farqlanadi.

Qo'shni populyatsiyalarni ayni tur yashashi uchun noqulay bo'lgan 
hududlar ajratib turadi. Jumladan, qora baliqning har bir buloqdagi populyatsiyasini quruqlik chegaralab turadi. Lekin, ayrim turlar keng hududlarda yoppasiga tarqalish asosida populyatsiyalarni hosil qilishi mumkin. Masalan, cho'l va chala cho'llarda yumronqoziqlarning miqdor zichliklari doimo yuqori bo'ladi, ayrim hollarda ular yashash uchun bir muncha noqulay joylarni ham mavsumiy - vaqtincha egallab oladi. Yumronqoziqlar populyatsiyalari o'rtasidagi chegaralar shartli ravishda miqdor zichliklari asosida faqlanadi.

Turning yoppasiga tarqalishiga barcha biotop va mintaqalarda ko'plab uchraydigan etti nuqtali xonqizi qo'ng'izi ham misol bo'ladi. Ular qishlash oldidan ancha uzoq masofalarga migratsiya qiladi. Bunday holatda populyatsiyalar o'rtasidagi chegaralar sust farqlanadi. Bir biotop va yaqin hududlarda yashayotgan qo'ng'izlar uzoq mintaqalardagi vakillariga nisbatan ko'proq aloqada bo'ladi. Ayni vaziyatda masofada, individlar o'rtasidagi aloqani cheklab populyatsiyalararo chegarani ifodalashi mumkin.

Ba'zida bir turning o'zi aniq ifodalangan va chegaralari aniq ifodalanmagan populyatsiyalarga ega bo'ladi. Boshoqli ekinlarning havfli zararkunandalaridan biri hasvalar tuxumidan rivojlanib chiqqandan so'ng 22,5 oy davomida jadal ovqatlanadi. Shundan so'ng ular qishlash uchun ixota daraxtzorlari yoki tog' oldi o'romonzorlari tomon yuzlab, ayrim hollarda esa minglab kilometr masofaga uchib ketadi, daraxt va butalar ostidagi shoxshabba to'kilgan barglar orasida bahorga qadar tinim davrida bo'ladi. Hasvalarning masofa bo'ylab uchish uzoqligi tanasiga yig'ilgan yog' zahirasiga bog'liq. Natijada qishlash joylarida turli hududlardan uchib kelgan qandalalar bo'lishi mumkin. Qishlab chiqqan qandalalar bahorda shamol yo'nalishiga monand tarqaladi va kelgan manzillariga qaytishi shart emas. Shu asosda keng hududlar bo'ylab hasvalarning doimiy aloqasi va almashinishi ro'y beradi. Bu holat populyatsiyalarni aniq chegaralanishiga to'sqinlik qiladi. Hasvalar ayrim tog' oldi hududlarida muqim yashaydi va aniq chegaralangan populyatsiyalarni hosil qiladi.

Populyatsiyalar o'rtasida individlar almashinuvi doimiy yoki vaqti-vaqti bilan sodir bo'lishi mumkin. Quzg'unlar mavsumiy ko'chish davrida galadagi yosh quzg'unlarning bir qismi shu joyning o'zida qolib muqim yashayotgan vakillari bilan yangi juftliklarni shakllantiradi. Qo'shni suv havzalaridagi 
baliqlar o'rtasidagi individlar almashinuvi juda kam ro'y beradi. Bahorgi suv toshqinlari tufayli baliqlar o'zi yashab turgan joydan ikkinchisi yashab turgan joyga o'tishi mumkin.

Populyatsiyalar o'rtasidagi aloqalar turni yagona birlik sifatida barqaror mavjud bo'lishini ta'minlaydi. Aksincha, populyatsiyalarni bir-biridan keskin chegaralanishi yangi turlarning hosil bo'lishiga sabab bo'ladi.

Populyatsiyalar o'rtasidagi farqlar faqat miqdoriy ko'rsatkichga asoslanmay, ulardagi morfologik, fiziologik va fe'l-atvor belgilarini o'zida ifodalashi mumkin. Populyatsiyalar o'rtasidagi farqlarning barchasi tabiiy tanlanishning mahsuli sifatida yuzalanadi. Qozog'istonda dala sichqonlari tog' daryolari va suv yo'llari bo'ylab yashashga moslashgan, uning past tekislikdagi vakillari yopiq suv havzalarining zich qamishzor va to'g'ayzorlarida hayot kechiradi. Tog'da tarqalgan sichqonlar kurak tishlarining o'tkirligi, dumining kaltaligi bilan ajralib turadi. Past tekisliklardagidan farqliroq ular qishga ozuqa yig'adi va bir yilda 6-7 oy davomida ko'payadi. Past tekisliklarda esa dala sichqonlari yil davomida ko'payadi, har bir urg'ochi tog'dagilarga nisbatan 1-2 ta ko'p bola berishi mumkin.

N.P.Naumov ta'rifiga ko'ra, tur - bir-biriga bosqichma-bosqich bo'ysinuvchi ierarxik tizimdagi populyatsiyalar majmui. Eng yuqori bosqichda turning kenja turlari yoki geografik farqlari turadi. Kenja turlarning tarqalish kengligi turning biologik xususiyatlariga bog'liq bo'ladi. Faol harakatlanuvchi turlar va kenja turlarning areallari juda keng bo'lishi mumkin. Ayni mintaqaning geografik shart-sharoitlari yaqin bo'lgan hududlarini geografik populyatsiyalari egallaydi. Geografik populyatsiyalar, o'z navbatida, ayrim joylarni egallovchi ekologik, biotopik, mahalliy, elementar populyatsiyalardan tashkil topadi. Quyi bosqichga tomon populyatsiyalar o'rtasidagi munosabatlar kuchayib, ular o'rtasida farqlar kamayib boradi. Yuqoriga tomon populyatsiyalararo munosabatlar chegaralanib, kenja tur doirasidagi morfologik, fiziologik farqlar va o'ziga xos fe'l-atvor belgilari barqarorlashib boradi.

\section{Populyatsiyalarning biologik tuzilmasi}

Populyatsiya tuzilmasining ko'rsatkichlari - miqdor zichligi, 
organizmlarning kenglikdagi taqsimlanishi, turli yosh va jinsga mansub individlarning nisbati bilan ifodalanadi.

Organizmlarning individual xususiyatlari genotipga va undan mujassamlangan irsiy axborotni ontogenezda namoyon bo'lishi va yuzalanishiga bog'liq. Har bir individ jinsi, o'lchamlari, fe'l-atvori, morfologiyasi va muhit omillariga moslanuvchanlik darajasi bilan farqlanadi. Mazkur belgilarning taqsimlanishi ham populyatsiya tuzilmasini ifodalaydi.

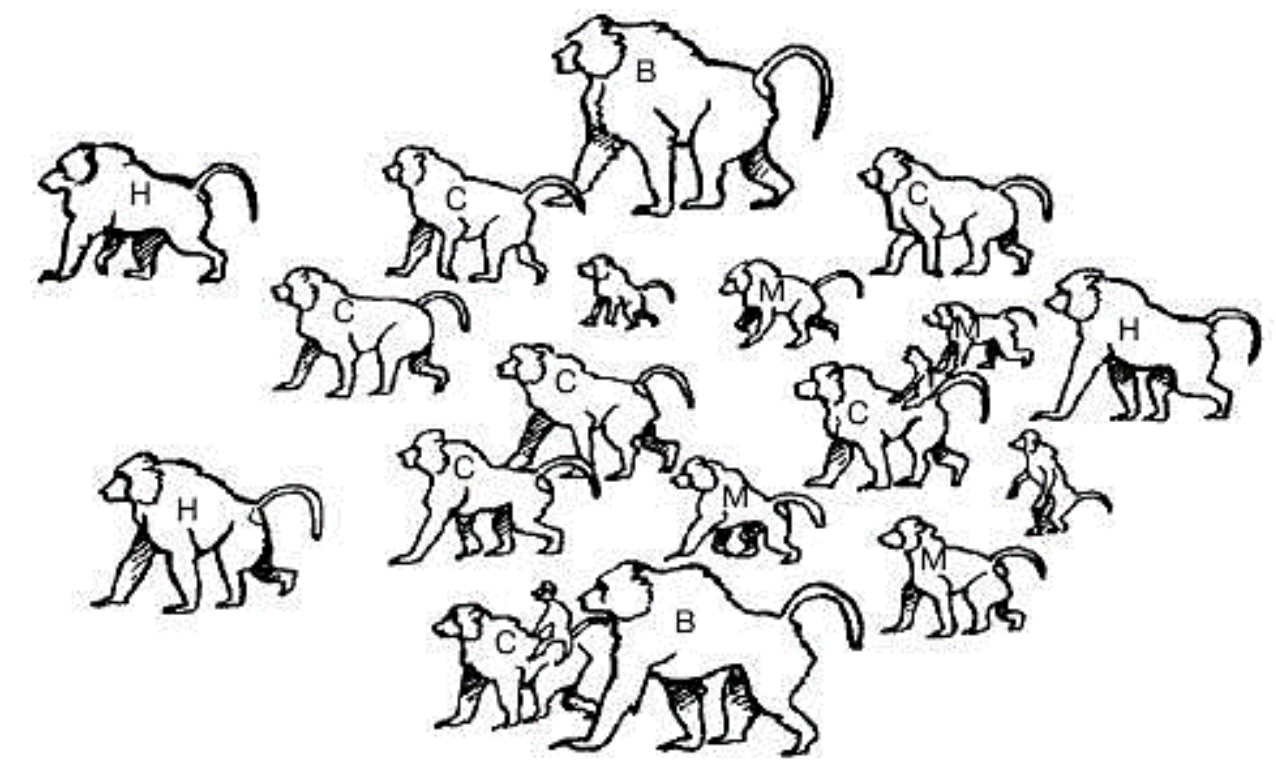

11-rasm. Pavianlarda to'da shakllanishi: S - urg'ochi; M - bolalari; V - to'da boshi ; N past darajadagi (yoki kichik) erkaklar

Populyatsiya tuzilmasi barqaror emas, u o'zgaruvchan. Organizmlarning o'sishi va rivojlanishi, tug'ilishi va nobud bo'lishi miqdor zichligining yuqorilashishi va pasayishi populyatsiya doirasidagi turlar nisbatlarining o'zgarishiga sabab bo'ladi. Ayni davrdagi populyatsiya tuzilmasi bundan keyingi o'zgarishlar yo'nalishlarini belgilaydi.

Populyatsiyaning ko'rsatkichlari. Populyatsiyadagi individlar mo'lligi uning muhim ko'rsatkichlaridan bo'lib sonlarda, absolyut va nisbiy zichliklarda ifodalanadi. Aniq chegaraga ega bo'lgan populyatsiyalardagi individlarning umumiy sonini aniqlash va belgilash birmuncha qulay. Boshqa holatlarda popuyatsiyaning zichligi aniqlanadi. Populyatsiya zichligi ma'lum maydon birligi $\left(1 \mathrm{sm}^{2}, 1 \mathrm{~m}^{2}, 1 \mathrm{~km}^{2}\right.$ va boshqalar) ga to'g'ri keluvchi individlar sonidir. Populyatsiyaning zichligi va miqdori bir-biriga bog'liq ko'rsatkichlardan, populyatsiyalar tadqiq etilayotganda turlar miqdori va zichligi barobar e'tiborga olinadi. Ta'kidlanganidek, populyatsiyaning absolyut 
va nisbiy zichligi farqlanadi. Absolyut zichlik ma'lum bir maydon birligiga to'g'ri keluvchi populyatsiyaning miqdorini ifodalaydi, ma'lum maydon birligidagi individlar soni zichlikka mos keladi. Populyatsiyalar qiyoslanayotganda ketma-ket kuzatishlarda ulardagi nisbiy zichlik e'tiborga olinadi. Nisbiy zichlikning o'zgarishi asosida populyatsiyadagi ko'payish yoki kamayishi vaqt birligidagi ko'rsatkichlarini aniqlash mumkin.

Quruqlik hayvonlarning populyatsiyalaridagi individlar soni maydon birligida, suv - ko'pchilik umurtqasizlar, tuproq, mezo va mikrofaunasi suv va tuproqning hajm birligida hisobga olinadi. Populyatsiyaning zichligi vaqt birligi davomida o'zgarib turadi. Populyatsiya zichligining o'zgarishi cheksiz bo'lmaydi.

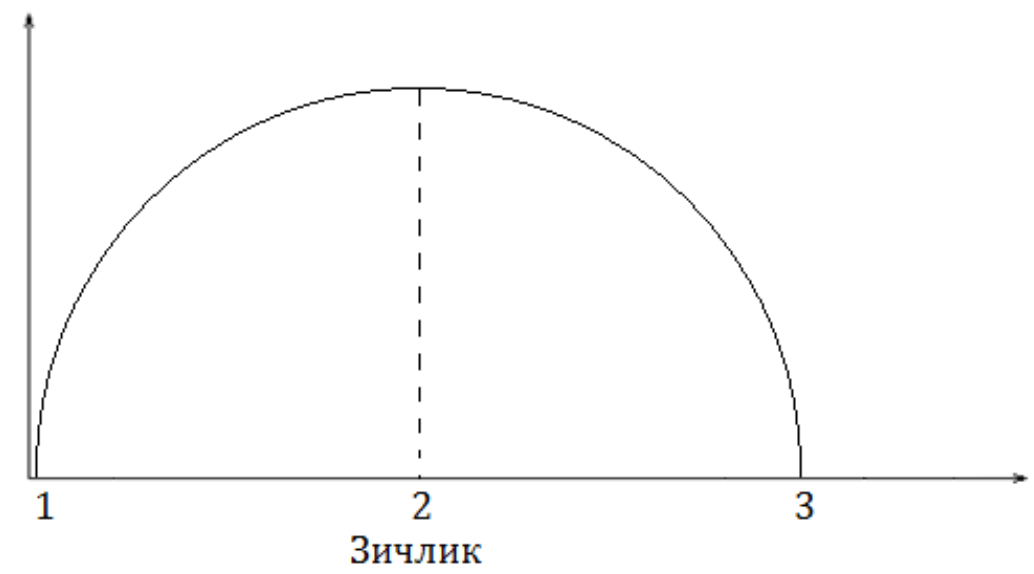

12-rasm. Olle qoidasining tasviri

Populyatsiya zichligining quyi chegarasi undagi individlar sonining minimal darajada bo'lishini, shuningdek, populyatsiyaning bundan keyingi ijobiy yoki salbiy taqdirini ham ifodalaydi. Zichlikning yuqori chegarasi populyatsiyadagi individlar sonining ortib ketishi, tur ichidagi munosabatlarning kuchayishi va boshqaruvchi omillar hisobiga o'z-o'zini boshqarish, cheklash holatini ifodalaydi. Populyatsiya zichligi o'rtacha ko'rsatkichga ega bo'lganda, individlar hayotchanligi eng yuqori darajada bo'ladi. Bunda barcha hayotiy jarayonlar yuqori samarali bo'lib, populyatsiyadagi individlarning yashovchanligi, ko'payishi va rivojlanishi uchun qulay sharoit yuzaga keladi. Ushbu holat ekologiyada Olle qoidasi asosida izohlanadi (12-rasm).

Populyatsiyaning jins tuzilmasi. Populyatsiyaning jins tuzilmasi erkak va urg'ochi individlarning son jihatdan nisbatidir. Jinslar nisbati 
organizmlarda ko'payishning dastlabki bosqichlarida - otalanishda aniqlangan bo'ladi. Xromosomalar o'rtasidagi mutanosiblik va belgilarning almashinishi bo'lajak avlodning morfologiyasi, fiziologiyasi va fe'l-atvorini belgilaydi.

Populyatsiyaning o'lchami. Har xil turlarning populyatsiyalari birbiriga o'lchami bilan farq qiladi. Tur individlar qanchalik yirik bo'lsa, populyatsiya areallari keng va bir muncha siyrak tarqalgan, aksincha mayda turlar kichik maydonda yoki yuzada zich populyatsiyalarni hosil qiladi. Ushbu holat populyatsion tuzilmasi mavzusida batafsil ko'rib o'tilgan.

Populyatsiyaning miqdor zichligini o'zgarishida jinslar bir-biriga teng bo'ladi. Aksariyat hollarda qaysidir jins ulushi yuqoriroq bo'ladi. Ekologik nuqtai nazardan populyatsiyadagi jinslar nisbatining biror tomonga siljishi ushbu populyatsiyaning mavqei bilan bog'liq. Masalan, asalarilar, termitlar va chumolilarda erkaklari faqat otalanishda ishtirok etadi. Shu sababdan jamoadagi urg'ochi jinslar nisbati ko'p bo'ladi. Ko'pchilik umurtqali hayvonlar ontogenezining dastlabki bosqichlarida erkaklari ko'proq uchraydi.

Ondatralar populyatsiyalarida yangi tug'ilgan bolalari orasidagi erkak va urg'ochilari soni deyarli teng bo'ladi, keyinchalik esa urg'ochilar soni bir muncha pasayib ketadi va ular o'rtasidagi nisbat 140:100 ni tashkil etadi. Tovuqsimonlar va o'rdaksimonlarda ham xo'rozlari ko'proq bo'ladi.

Bolalariga ozuqa topish va ularni katta qilishda erkak va urg'ochilari bir xil ishtirok etadigan hayvonlar populyatsiyalaridagi jinslar nisbati teng bo'ladi. Jumladan, monogam sut emizuvchilar, qushlar va boshqalar shular jumlasidandir. Populyatsiyaning barqarorligida urg'ochi organizmlar soni muhim ahamiyatga ega. Jinslar nisbatiga tashqi muhit omillari ham ta'sir etadi. Masalan, o'rmon chumolilari tuxumidan Q200 S li haroratdan past sharoitda faqat erkaklari, yuqori haroratda esa urg'ochilari rivojlanishi kuzatilgan. Buning asosiy sababi tuxum yo'lidagi spermalar faqat yuqori haroratli sharoitda faol bo'lib, tuxumni otalantira olishidir. Pardaqanotlilarning otalanmagan tuxumlaridan erkaklari rivojlanadi. Shiralarning erkak va urg'ochilari kech kuzdagina paydo bo'ladi (rivojlanadi), ya'ni bu jarayonga harorat, kun uzunligining o'zgarishi ta'sir etadi.

Populyatsiyaning yosh tuzilmasi. Organizmlarning tug'ilish va o'lim (nobud bo'lish) o'rtasidagi mutanosiblik jinslar o'rtasidagi nisbat bo'lib, populyatsiyaning ko'payishi - reproduktsiya imkoniyatini belgilaydi. 
Hayvonlar hayotida 3 davr farqlanadi. Jinsiy voyaga etmagan (yosh bolalik), jinsiy etuklik va qarilik davrlari. Har xil turda bu ko'rsatkich turli xil bo'lishi mumkin. Ko'pchilik hayvonlarda birinchi davr ancha uzoq bo'ladi. Masalan, kunliklarning lichinkalari 1-3 yil umr ko'radi, imago davrida 2-3 soat, ba'zan bir necha kun yashaydi xolos. Shuningdek, bahorilar voyaga etgan davrida oziqlanmaydi, bir necha lichinkasi esa suvda 1-3 yil hayot kechiradi. Amerika tsikadasining lichinkalik davri 17 yil davom etadi.

Yosh piramidalari 3 turga bo'linadi. Keng asosga ega bo'lgan yosh piramidalar jadal ko'payish xususiyatiga ega bo'lgan hayvonlarda kuzatiladi. Ularning populyatsiyalarida bolalari soni doimo yuqori bo'ladi. O'rtacha asosga ega bo'lgan yosh piramidalar me'yorida ko'payadigan hayvonlar populyatsiyalariga xos. Tor asosga ega bo'lgan yosh piramidalar xos bo'lgan populyatsiyalardagi tug'ilish ancha past darajada, qarilarning soni ortiq bo'ladi.

Hayvonlar populyatsiyalarining yosh tuzilmasiga antropogen omil ham ta'sir etishi mumkin. Jumladan, ov qilinadigan hayvonlarning yosh nisbatlari imagolarning xo'jalik faoliyati natijasida o'zgarishi kuzatiladi. Masalan, eng ko'p ovlanadigan baliqlardan seldsimonlarning 5-6 yoshdagilarigina to'rga tushadi. Yoshlari to'r teshiklaridan bemalol o'tib ketadi.

Turning ko'payish xususiyatlariga bog'liq holda populyatsiya bir bo'g'inga yoki bir necha bo'g'inga mansub individlardan tashkil topadi. Bahorda chigirtkalarning qishlab chiqqan tuxumlaridan birinchi yoshdagi lichinkalar paydo bo'ladi. Mikroiqlimga bog'liq holda lichinkalarning tuxumdan rivojlanishi bir tekis ketmasligi mumkin, lekin ma'lum davr oralig'ida ularning barchasi tuxumdan chiqib ulguradi. Bu davrda mazkur populyatsiya faqat birinchi yoshdagi lichinkalardan iborat bo'ladi. Keyingi 2-3 hafta davomida individlarning rivojlanishidagi mutanosiblikning buzilishi sababli populyatsiyada turli yoshdagi lichinkalar uchraydi. Yoz oxirida esa barcha lichinkalar voyaga etib ulguradi va populyatsiya to'lig'icha jinsiy etuk individlardan tashkil topadi. Ayrim hayvonlarning populyatsiyalari tarkibida turli bo'g'inga mansub individlar uchraydi. Bu kabi hayvonlar 2 guruhga ajratiladi: hayoti davomida bir marta va ko'p marta ko'payadiganlar.

May qo'ng'izining urg'ochilari tuxum qo'ygandan so'ng tez nobud bo'ladi. Uning lichinkalari 3 yil hayot kechirib, keyin g'umbakka aylanadi va 
undan etuk hasharot chiqadi. May qo'ng'izining populyatsiyasida bir davrning o'zida 4 yoshga mansub individlar uchraydi. Har yili oxirgi yoshdagilari voyaga etib hayotini tugallaydi, yangi bo'g'in individlari paydo bo'ladi.

Ko'payishi may qo'ng'iziga o'xshash hayvonlarning bir necha avlodi mavsum davomida almashishi mumkin. Masalan, g'o'za tunlami g'umbak holda qishlaydi va mavsum davomida 3-4 avlod beradi. Ularning populyatsiyalarida, ayrim hollarda ikki yoki uch bo'g'inga mansub individlar uchrashi mumkin.

Hayoti bir necha marta ko'payish xususiyatiga ega bo'lgan hayvonlar populyatsiyalarining yosh tuzilmalari ancha murakkab bo'ladi. Jinsiy voyaga etgan davri qisqa bo'lgan hayvonlarning populyatsiyalaridagi yosh nisbatlari barqaror bo'lmaydi. Dala sichqonlari populyatsiyalarining yosh tuzilmasi mavsum davomida murakkablashib boradi. Bahorda populyatsiya o'tgan yilda tuzilgan individlardan tashkil topadi. Keyin ularga birinchi va ikkinchi bo'g'in vakillari qo'shiladi, 3 va 4-bo'g'in dunyoga kelayotgan davrda dastlabki ikki bo'g'inga mansub sichqonlar voyaga etib bola beradi. Kuzga borib populyatsiyani bahorda tashkil etgan etuk sichqonlar nobud bo'ladi va populyatsiya turli yoshga mansub individlardan iborat bo'ladi.

Uzoq umr ko'ruvchi hayvonlar populyatsiyalaridagi yosh nisbati barqaror bo'ladi. Jumladan, Hindiston fillari 60-70 yil umr ko'radi, 8-12 yoshida jinsiy voyaga etadi. Urg'ochi fil 4 yilda bir marta bir yoki ikkitadan bola tug'adi. Ularning populyatsiyalaridagi voyaga etgan individlar soni $80 \%$, bolalari soni esa $20 \%$ ni tashkil etadi. Populyatsiyadagi bu holat deyarli barqaror holda saqlanadi.

Populyatsiyalarning fazoviy tuzilmasi. Populyatsiya egallab turgan kenglik individlar uchun oziqlanish va yashash joyi. Populyatsiyaning fazoviy tuzilmasi populyatsiya maydonidagi ayrim individlarning tarqalish xususiyatini ifodalaydi. Ma'lum hudud muayyan sondagi individlarni oziqlantira oladi. Shuning uchun ham tabiiy resurslardan oqilona foydalanish nafaqat populyatsiya zichligiga, balki individlar va guruhlarning kenglik bo'ylab taqsimlanishiga ham bog'liq. Odatda tur va populyatsiyalar ichida individlar bir tekis tarqalmaydi, zero, yashash sharoiti, ozuqa manbalari barcha joyda birdek emas.

Hayvonlarning o'ziga xos biologik xususiyatlari, harakatchanligi sababli 
ulardagi hududiy munosabatlari o'simliklarga nisbatan ham turli-tuman bo'ladi. Hatto o'troq yashovchi hayvonlarda ham hududiy munosabatlar farqlanadi. Jumladan, astsidiyalar koloniyalari kattalashib borib qo'shni koloniyalarni surib chiqarish kuzatiladi. Shuningdek astsidiyalar koloniyalari o'zining turdosh koloniyalari bilan to'qnash kelganda ularni chetlab o'tish va yo'nalish bo'ylab siljishi mumkin. Ko'pchilik hollarda ayrim hayvonlar koloniyalarni keng doiradagi yashash joylarini egallab olgach, vegetativ ko'payishdan jinsiy ko'payishga o'tadi. Rivojlanib voyaga etgan harakatchan lichinkalar erkin harakatlanib yangi hududlarga borib o'rnashadi va yangi koloniyalarni hosil qiladi.

Populyatsiyalarni tashkil etuvchi individlarning fazoviy taqsimlanishi turli xilda bo'lishi mumkin. Ularning fazoviy taqsimlanish xususiyatlarini tahlil etish populyatsiya zichligini baholash va izohlash imkoniyatini beradi. Bunda saylanma namunalar olish usuli qo'llaniladi. Agar saylanma namunalar soni $n$, har bir namunadagi individlar sonining o'rtacha qiymati $m$ ga teng bo'lsa, taqsimlanish dispersiyasi yoki ko'rsatkichini $\left(\mathrm{S}^{2}\right)$ ushbu formula asosida hisoblab topish mumkin:

\section{$\mathrm{S}^{2} \mathbf{q} \sum(\mathrm{x}-\mathrm{m})^{2}$}

\section{$n-1$}

Agar individlar bir tekis tarqalgan bo'lsa, taqsimlanish disspersiyasi - $\mathrm{S}^{2}$ nolga, tasodifiy tarqalganda o'rtacha qiymat $-m$ ga teng bo'ladi. Individlar to'da-to'da bo'lib, guruhli tarqalgan o'rtacha qiymat ko'rsatkichi taqsimlanish disspersiyasi qiymatidan yuqori bo'ladi. Bu farq qanchalik kattalasha borsa, hayvonlarning guruhlar hosil qilib tarqalishga moyilligi yuqori bo'ladi.

Hayvonlarning bir tekis tarqalishi tabiatda kam uchraydi. Suv bo'yida yashovchi yirik koloniyali ayrim qushlarning individlari, yirtqich baliqlar yashash joylari bo'ylab bir tekisda joylashgan.

Tasodifiy tarqalishda individlar bir-biridan har xil masofada, tartibsiz joylashadi. Shiralar ozuqa o'simligida dastlab tartibsiz joylashadi. Hasharotlarning tuxumdan chiqayotgan lichinkalari tasodifiy tarqaladi.

Tabiatda guruhli tarqalish ko'p uchraydi. Bunda individlar to'da hosil qilib bir-biridan turlicha masofada joylashadi. Individlar to'da ichida bir tekis yoki joy egallashi mumkin. 

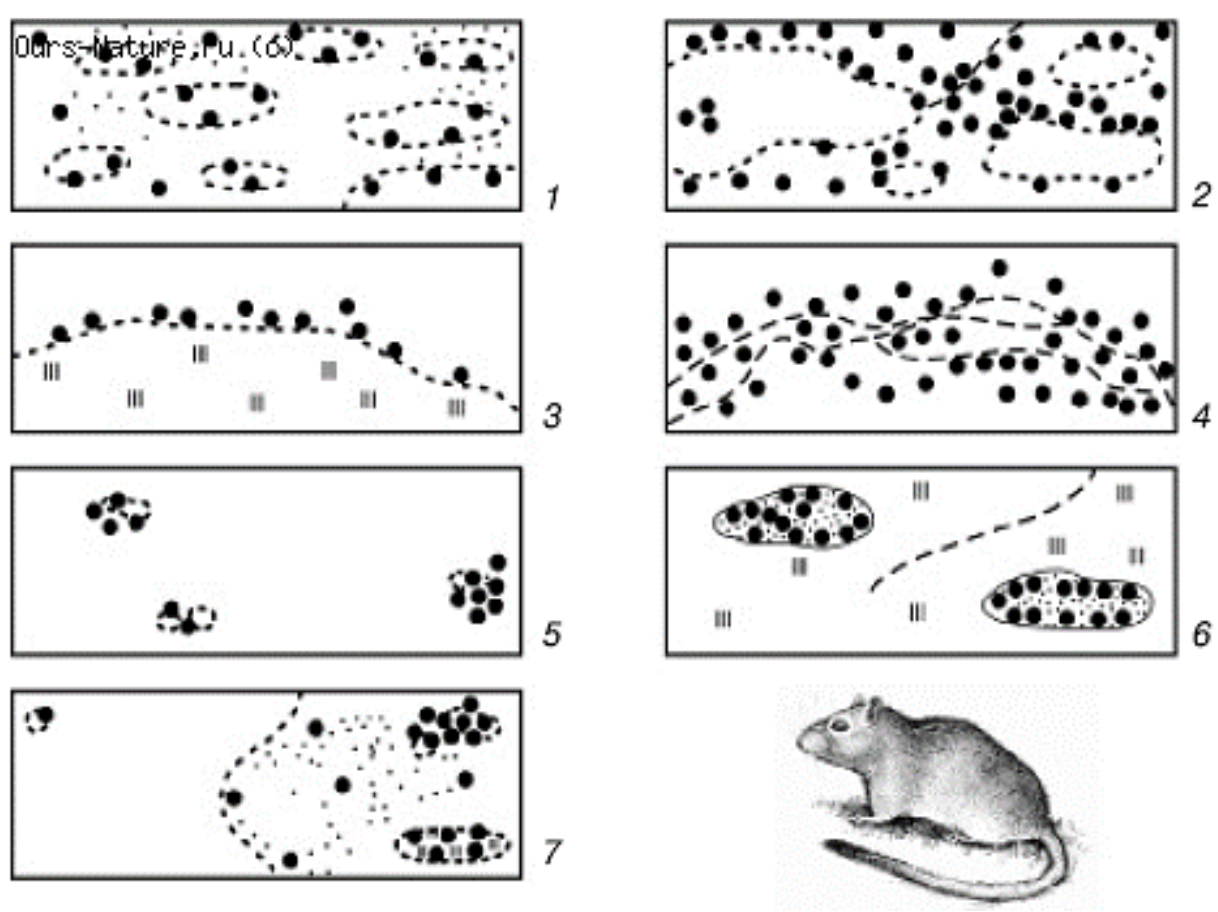

13-rasm. Individlarning fazoda tarqalishining asosiy ko'rinishlari (tasodifiy taqsimlanish qonuni)

Umurtqali hayvonlarning populyatsiyalar ichidagi taqsimlanishi instinktlar majmui yordamida boshqariladi. Ular uchun fe'l-atvor reaktsiyalari xos. Hududiy tarqalish sut emizuvchilar, qushlar, sudralib yuruvchilar, baliqlar va ayrim amfibiyalarda kuzatiladi. Umurtqasizlardan o'rgimchaklar va osminoglarda ham hududiy tarqalish xususiyatlari qayd etilgan.

Hududiy kenglikni ishg'ol qilish va undan foydalanish nuqtai nazardan hayvonlar 2 ta ekologik guruhga bo'linadi: doimiy - muqim yashovchi va ko'chmanchi hayvonlar.

Muqim yashovchi hayvonlar butun hayoti davomida yoki hayotining ma'lum bir davrida o'troq hayot kechiradi. Bu guruhga mansub organizmlar yashash joylariga o'ta bog'langan bo'ladi. Ushbu holat ekologiyada «xoming» (inglizcha "home" - "uy" so'zidan olingan) nomini olgan. Keyinchalik turlar uzoq muddatdan so'ng ham yashash joylariga qaytadi. Bu o'rinda qushlarning safar uchishlari misol bo'ladi. Kaptarlar uzoq masofa bo'ylab mo'ljalni to'g'ri oladi va uyini topib keladi.

Muqim yashash osonlik bilan, erkin mo'ljal oladi, ozuqa oson va tez topadi, qisqa muddatda yashirina oladi. Ular uya va inlar quradi, uyalarini bog'lovchi qo'shimcha yo'llarni ochadi. Olmaxonlar asosiy uyasida ko'payadi, 
qo'shimcha uyalarida ozuqa to'playdi, dushmanlaridan va noqulay obhavodan saqlanadi.

O'z hududidan chetga chiqqan hayvonlar qo'rqoq, bo'shashgan va himoyasiz bo'ladi. O'troq yashash biologik ustunlikka ega bo'lsa-da, populyatsiya zichligining jadal ortishi natijasida ozuqa resurslari chegaralanib qolishi mumkin. Muqim yashovchi hayvonlar individlari va guruhlari o'rtasida yashash joylari bo'ylab ma'lum masofa saqlanadi. Bu holat ozuqa resurslari va yashash joylaridan unumli foydalanish imkoniyatini beradi. Shu bilan birga, ular o'rtasidagi mustahkam aloqa saqlanib turadi.

Hayvonlarning hududiy fe'l-atvor reaktsiyalari har individning yashashini ta'minlash, ozuqa izlash, uya qurish, himoyalanishga sharoit yaratish, shuningdek, boshqa sheriklari bilan bo'ladigan aloqani o'rnatib turish (joyni qo'riqlash, signallar berish, belgilar qo'yish va boshqalar) uchun xizmat qiladi. Har bir individning yashash joyi etarli ozuqa resursiga ega va hududiy munosabatlar ta'minlangan bo'lishi zarur.

Hayvonlar o'z hududlarini to'g'ridan-to'g'ri agressiv yo'l bilan, qo'rqitish, kuchli ovoz chiqarish, maxsus signallar berish va turli belgilar qo'yish orqali qo'riqlaydi. O'z hududini qo'riqlayotgan hayvonlar ovozlar berish, turli belgilar qo'yish orqali qo'riqlaydi.

O'z hududini qo'riqlayotgan hayvonlar ovozlar berish, turli hattiharakatlar orqali ogohlantiradi, to'g'ridan-to'g'ri qarama-qarshilikka juda kamdan-kam chiqadi. Joyning egasi uchun himoya fe'l-atvori "begona" individ uchun tezroq uzoqlashish xususiyatiga xos.

Hayvonlar yashash joylarini turli belgilar qo'yish orqali chegaralab oladi. Qushlar sayrab joy egallanganligidan ogoh etadi. Sut emizuvchilar o'z hududi chegarasiga ekskrementlarini yoki maxsus bezlardan ajralayotgan hidli suyuqlikni qo'yib chiqadi. Maxsus bezlar sobollarning oyog'i tagida, kiyik echkilar shoxlarning orqa tomonida, antilopalarda esa ko'zining oldingi burchagida bo'ladi. Itsimonlar, mushuksimonlar va lemurlar siydigi yordamida belgi qo'yadi. Ayiqlar, zubrlar va yovvoyi mushuklar daraxtlarning tanasini chuqur o'yib iz qoldiradi.

Hayvonlarning hududiy fe'l-atvor harakatlari ko'payish davrlarida kuchayadi.

Ko'chmanchi qushlardagi hududiy harakatlar qishlash joylarida 
ifodalanadi. Yirtqich va mayda kemiruvchilar butun hayoti davomida muyayan joy bilan bog'langan bo'ladi. Barqaror oilaviy guruhlardagi hududiy munosabatlar bir muncha murakkab bo'ladi. Yo'lbarslar oilasida bir erkagi, 23 ta urg'ochi va bolalaridan tashkil topadi. Ularning oilaviy hududi o'ljaning ko'p ozligi bilan belgilanadi. 1000 gektarga 14-15 tadan tuyoqli hayvonlar to'g'ri kelgan taqdirda yo'lbarslarning oilaviy hududi 50000 gektarni tashkil etadi.

Ko'chmanchi hayvonlar doimiy harakatda bo'ladi, ular uchun ozuqa zahiralarini kamayishi muammosi bo'lmaydi. Ular podalar va galalar holida harakatlanadi. Yonma-yon harakatlanayotgan hayvonlar o'rtasida aniq masofa saqlanadi. Bu holat baliqlar va qushlarda aniq ifodalangan, ular o'rtasidagi aero va gidrodinamik sharoit to'liq saqlanadi. Ko'chmanchi hayvonlarning harakat yo'nalishi bo'ylab joylashgan dam olish joylari bo'ladi, ayrim hollarda ular qisqa muddat davomida o'troq hayot kechirishga o'tishi mumkin. Ko'chmanchi hayvonlarning harakat kengligi sharoitga bog'liq. Masalan, zebralar qurg'oqchilik mavsumida 400-600 $\mathrm{km}^{2}$ kenglikda harakatlansa, namlik etarli sharoitda $300-400 \mathrm{~km}^{2}$ kenglikda ko'chib yurishi mumkin.

\section{Populyatsiyaning ekologik tuzilmasi}

Etologiya (grekcha "ethos" - "xulq-atvor", "fe'l-atvor", "xarakter") hayvonlar fe'l-atvori, hatti-harakatlarining qonuniyatlari haqidagi fandir. Hayvonlarning fe'l-atvori va turli xatti-harakatlari orqali bo'ladigan aloqalar majmui etologik munosabatlar deb nomlanadi. Hayvonlarning fe'l-atvori ularning hayot tarzi bilan bog'liq. Hayvonlar odatda yakka-yolg'iz yoki birgalikda hayot kechiradi. Hayvonlar birgalikda yashashining bir necha shakllari ma'lum.

Yakka-yolg'iz yashash. Populyatsiyadagi ayrim individlar hayotining muyayan bir davrda yolg'iz yashaydi. Tabiatda hayoti davomida butunlay yolg'iz hayot kechiruvchi hayvonlar deyarli uchramaydi, nasl qoldirish uchun jinsiy yo'l bilan uchrashadi. Shuningdek, ayrim yolg'iz yashovchi hayvonlar ovqatlanish, qishlash davrida uchrashadilar. Ekologiyada yolg'iz yashash nisbiy ma'noda tushuniladi. Xon qizi qo'ng'izlari, yirtqich qo'ng'izlar yolg'iz hayot kechirsa-da, qo'shilish davrida, qishlash mavsumida yig'ilishi mumkin. 
Populyatsiyada individlar o'rtasidagi munosabatlarning murakkablashuvi, ularning jinsiy moyilligi, ota-onalar bilan yangi avlod o'rtasidagi bog'lanishlar natijasida hayvonlarning birgalikda yashash shakllaridan biri yuzaga keladi. Ota-onalar jufti qisqa yoki uzoq muddatli, ba'zi turlarda esa umrining oxiriga qadar bo'ladi. Tovuqsimonlar ko'pchiligi poligam qushlar bo'lib, barqaror oila hosil qilmaydi. Yovvoyi va suqur o'rdaklarning oilalari saxar uchish paytida shakllanadi va makiyonlari tuxum qo'ygunga qadar davom etadi, shundan so'ng xo'rozlari oilani tark etadi. Oqqushlar, turnalar va kaptarlarning oilalari ko'p yillar davomida saqlanib qoladi. Oilaning shakllanishi tur ichidagi qarama-qarshi munosabatlarning mahsuli sifatida yuzaga keladi. O'rgimchaklarning erkaklari qishlashi oldidan o'ziga xos harakatlar qilib, urg'ochisini "mahliyo" etadi. Qushlar chiroyli ovozlar chiqarib sayraydi, ba'zilari rang-barang patlarini namoyish etadi. Sut emizuvchilarning erkaklari o'rtasida o'zaro kuchli kurash boradi. Hayvonlardagi bu xatti-harakatlarning barchasi jinsiy tanlanish jarayonini muvofiqlashtirib, ikkala jins o'rtasidagi munosabatga tayyorgarlikni ta'minlaydi.

Koloniyalar (jamoalar) muhim o'troq yashovchi hayvonlarning o'ziga xos yig'inidir. Koloniyalar vaqtincha yoki doimiy bo'lishi mumkin. Ayrim hollarda ko'payish oldidan koloniyalar shakllanadi. Koloniyani tashkil etuvchi individlar o'rtasidagi o'zaro munosabatlar nihoyatda xilma-xildir.

Koloniyalar alohida hududdagi ayrim individlarning xemotaksis asosida oddiy yig'inidan boshlab, har biri alohida vazifani bajarishga ixtisoslashgan yagona organizm darajasida bo'lishi mumkin. Sifonoforalar va gidromeduzalar koloniyalaridagi har bir individ o'ziga xos vazifani bajaradi. Chag'alaylar, kayralar va g'ozlar koloniyalari ancha murakkab bo'lib, ayrim xattiharakatlarni ular o'zaro kelishib amalga oshiradilar, bu esa alohida olingan har bir individning xavfsizligini ta'minlaydi. "Qushlar bozori"dagi barcha parrandalar dushmandan saqlanish uchun zarur bo'lgan ogohlantiruvchi signal asosida yoppasiga harakat qilib, oq tulki, burgut kabi yirik yirtqichlar hujumini ham bartaraf etadilar. Qushlar koloniyalari egallagan hududda har bir individning xususiy joyi aniq bo'ladi. Masalan, baliqchi qushlarning uyalari orasidagi masofa 3-5 metr atrofida bo'ladi.

Sut emizuvchilardan sug'urlar, viskachalar, dumsiz sichqonlar, 
olasichqonlar, qumsichqonlar kolonial holda hayot kechiradi. Sut emizuvchilar orasida koloniya bo'lib hayot kechirish oilaviy guruhning kengayishi hisobiga kelib chiqadi va dastlabki oila bilan bog'lanishni saqlab qoladi.

Hasharotlar (termitlar, chumolilar, arilar) jamoalarida murakkab munosabatlar kuzatiladi, ularning asosida ham oilalarning kengayib ajralib chiqishi yotadi. Ularning koloniyalarida ko'payish, himoyalanish, bolalari va o'zlarini boqish, qurilish ishlari kabi vazifalar bir muncha oson amalga oshadi. Mehnat taqsimoti ayrim individlarning yosh xususiyatlarini hisobga olgan holda yoki ularni alohida vazifalarini bajarishga ixtisoslanishi asosida bo'ladi. Koloniyadagi har bir harakat a'zolari o'rtasidagi mustahkam aloqa asosida amalgan oshadi.

Chumolilarning koloniyalari bir yoki bir necha uyalarida joylashadi, uyalar o'rtasida bog'lovchi yo'lakchalar bo'ladi. Chumolilar birgalikda yashashida turli xildagi signallar tizimidan muntazam foydalanadi.

To'dalar yoki galalar hayvonlarning mavsumiy yig'inlaridir. Ayrim guruh hayvonlar biron bir biologik foydali xatti-harakatlarini amalga oshirishi uchun kunning ma'lum bir vaqtida yoki mavsumning bir davrida galalar hosil qiladi. To'dalar yoki galalar hayvonlarning dushmandan himoyalanish, o'ljani ta'qib qilish va ushlash hamda mintaqaviy harakatlarni engillashtiradi. To'da bo'lib yashash baliqlar, qushlar, sut emizuvchilardan itsimonlarda uchraydi. To'dada sherigiga ta'sir qilish, o'rnak olish va mo'ljal olish ko'p uchraydi.

Harakatlarning muvofiqlashtirilishiga ko'ra to'dalar 2 guruhga ajratiladi: alohida a'zolari ustunlik qilmaydigan va boshliq (lider)ga ega bo'lgan to'dalar. 


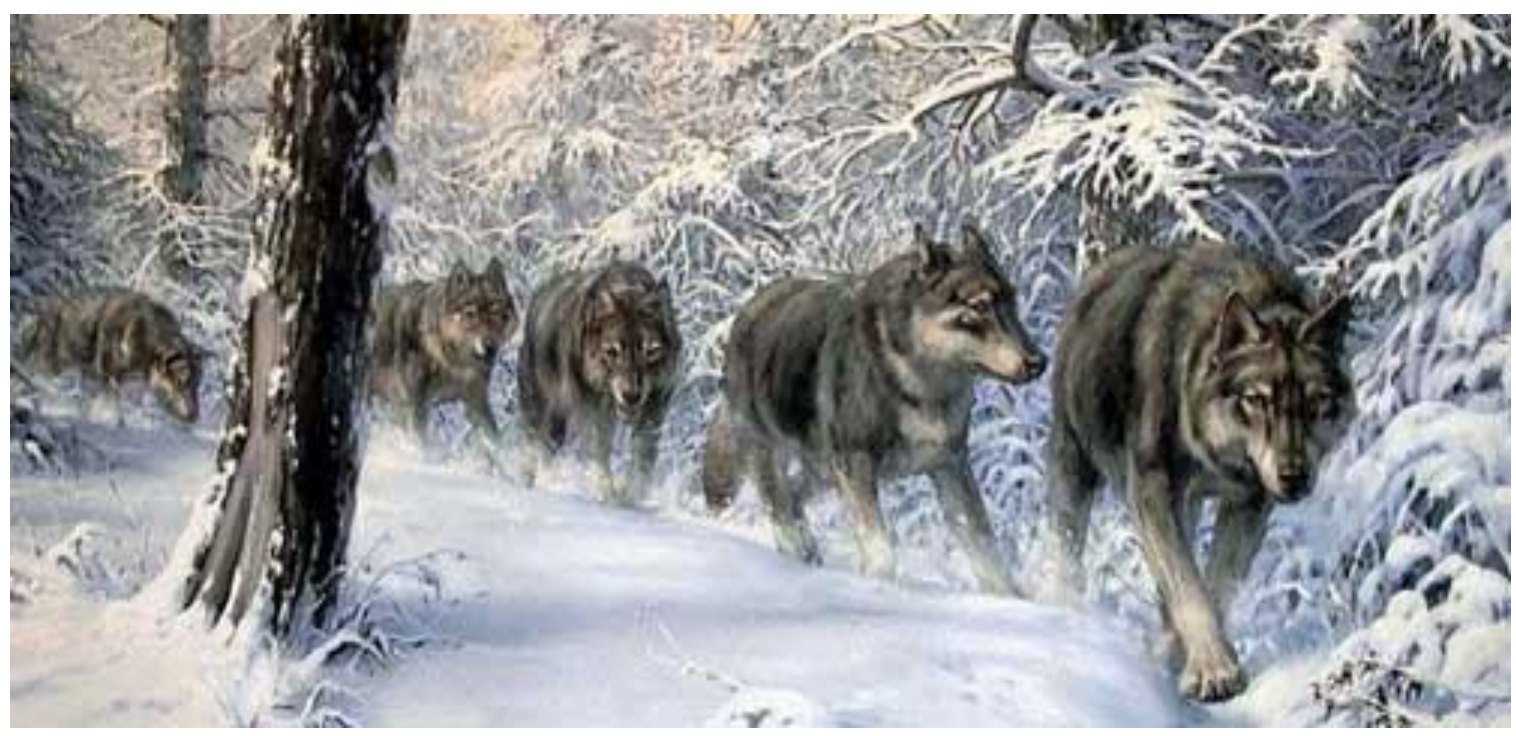

14-rasm. Liderga ega bo'lgan to'dalar

Birinchi guruhga mansub to'dalar baliqlar, mayda qushlar va chigirtkalar uchun xos.

Yirik qushlar va sut emizuvchilarda esa ikkinchi guruhga mansub to'dalar kuzatiladi. Baliqlar galasi shakli, kattaligi va zichligi jihatidan o'zgaruvchan bo'ladi, aksariyat hollarda kunduz kuni shakllanadi va kechalari tarqab ketadi.

Baliqlar galasi oziqlanishi, dushmandan himoyalanishi va migratsiyalarida asosiy ahamiyatga ega. Qushlar safar uchishlari va mavsumiy ozuqa topish va oziqlanish davrida galalar holida harakatlanadi. O'troq holda yashovchi qushlar o'rtasida doimo tovush chiqarib turish, ko'rish bilan bog'liq signallar bo'lsa, bo'rilar galalari qish mavusmida o'ljani ta'qib etish va ushlash uchun shakllanadi. Gala bo'ylab yashash bo'rilar, sirtlonlar, ko'klamzor bo'rilarida ham kuzatiladi.

Podalar galalarga nisbatan uzoq muddatli birlashish shaklida shakllanadi. Podada ozuqa topish, himoyalanish, migratsiyalar, ko'payish va bolalarni tarbiyalash kabi qator funktsiyalar amalga oshadi. Podadagi hayvonlar o'rtasida "hukmdor" va "itoatkor" munosabatlar amal qiladi. Podaning "hukmdori" vaqtincha yoki doimiy bo'lishi mumkin va uning xattiharakatlariga qarab boshqa individlar bir joyga joylanadi, mintaqaviy harakatlanishini, oziqlanish joyini hamda dushmanga munosabatni mo'ljalga oladi. Podadagi har bir individ liderga itoat qilga holda bir jon-bir tan bo'lib harakat qiladi. Rahbarning faoliyati "itoatkor"larni to'lig'icha bo'ysundirishga 
qaratilmagan. Tajribali va kuchli individ boshqaradi. Shimol bug'ulari podalariga bir muncha yoshi katta individlar boshchilik qilsa, fillar podasida urg'ochilari etakchi bo'ladi. Podaning "hukmdori” turli sonda bo'lishi mumkin. Jumladan, shimol bug'ulari podasida $18-20 \%$ ga qadar liderlar bo'lishi mumkin.

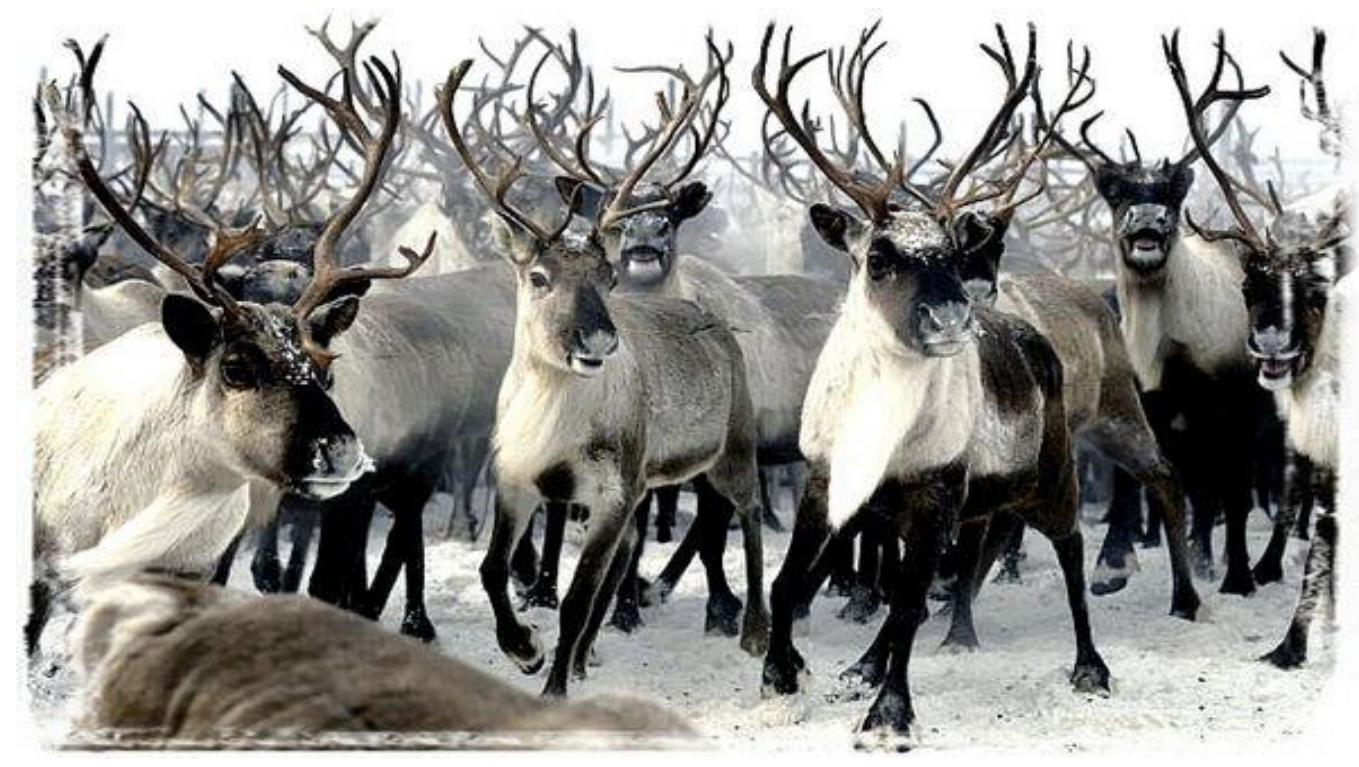

15-rasm. Shimol bug'ulari

Podaning biologik ahamiyati shundan iboratki, poda boshining tajribasi, tadbirkorligi va quvvati guruh hayotida etakchi ahamiyatga ega bo'ladi. Ular podani dushmandan himoya qiladi, uni etaklaydi, ovqatlanish va dam olishni ta'minlaydi, bolalarni tartibga soladi, janjallarni bartaraf etadi.

\section{Populyatsiyalar sur'ati}

Tabiatdagi barcha populyatsiyalar yirik omillari cheklanmagan taqdirda cheksiz ko'payishi mumkin va bu holat turning biotik potentsialiga bog'liq. Biotik potentsial tushunchasi ekologiyaga R.Cheplin tomonidan 1928 yilda kiritilgan. Bu ko'rsatkich urg'ochi organizmning hayot davomida yoki muayyan vaqt birligida nasl bera olish imkoniyatidir. Barcha turlarning biotik potentsiali bir xil darajada emas. Masalan, ayrim hayvonlar yillik hayoti davomida 10-15 marta bola berish mumkin. Asalarining biotik potentsiali 4050 mingga etadi. Ayrim hayvonlarning biotik potentsiali aslida bundan ham yuqori, lekin ularning nasli embrional taraqqiyoti dastlabki bosqichlaridayoq ko'proq nobud bo'ladi. Populyatsiya miqdor zichligining o'zgarishi qator 
omillarga bog'liq. Jumladan, populyatsiya sur'atini tug'ilish, o'lim, emmigratsiya va immigratsiya kabilar belgilaydi. Populyatsiyadagi tug'ilish darajasidagi muayyan vaqt davomida dunyoga kelgan individlar soni bilan ifodalanadi. Tug'ilish darajasi populyatsiyadagi jinsiy etuk individlar soni va jinslar nisbati birinchi navbatda ta'sir etadi. Shuningdek, u turning nasl berish tezligiga bog'liq. Hasharotlarda yil davomida berayotgan avlodlar soniga muvofiq mono va polivolt turlar farqlanadi. Monovolt hasharotlar mavsum davomida bir marta avlod beradi (masalan, may qo'ng'izi). Polivoltlar esa shu muddatda bir necha marta ko'payadi. Masalan, yashil olma shirasi bahorda kech kuzga qadar 13-15 avlod berishi mumkin. G'o'za tunlami avlodlari soni 3-4 taga etadi. Hayvonlar hayoti davomida bir marta yoki ko'p marta ko'payishi mumkin. Shunga muvofiq mono va politiklik turlar farqlanadi. Monotiklik hayvonlarning jinsiy voyaga etgan davri juda qisqa bo'ladi. Bahorilar, kunliklar, etuklik davrida 2-3 soatdan bir necha kunga qadar yashaydi, uchib suvga tuxum qo'ygach, nobud bo'ladi. Politsiklik turlar hayoti davomida bir necha bor ko'payadi. Umurtqalilar, umurtqasizlardan qisqichbaqasimonlar politsiklik hayvonlardir. Hindiston fillari 8-12 yoshida jinsiy voyaga etadi va 60-70 yillik umrining har 4 yilida bir yoki ikki marta bola beradi. Hatto, shu holatda ham bu hayvonlarning populyatsiya zichligi bir me'yorida saqlanadi.

Tug'ilish darajasiga organizmlarning umri davomida nasl bera olish davri sezilarli ta'sir etadi. Masalan, meva pashshalarining nasl berish davri umrining 65\% ni tashkil etadi. Ayrim chigirtkalar hayotining 15\%, kunliklar 0,5-1\% davrida avlod beradi.

Tug'ilish darajasini ifodalashda turning serpushtligi ham alohida o'rin tutadi. Surpushtlik darajasi har bir turning mavjudligini ta'minlovchi tarixiy evolyutsion moslanishdir. Muhit omillarining o'zgarishiga chidamsiz bo'lgan turlarning serpushtligi doimo yuqori bo'ladi, ular qisqa muddatli qulay sharoitda ko'plab nasl qoldirishga ulguradi, lekin qoldirgan avlodning ko'pchiligi rivojlanish davrining dastlabki bosqichlaridayoq qirilib ketadi. Foydali hasharotlarga nisbatan fitofaglarning serpushtlilik darajasi doimo yuqori bo'ladi. Masalan, kuzgi tunlam kapalagi 2000 dan ortiq, etti nuqtali xonqizi qo'ng'izi esa 700 ga yaqin tuxum qo'yishi aniqlangan. Qulay ekologik sharoitda yashaydigan turlarning serpushtliligi ancha past bo'ladi. Tropik 
turlarga qaraganda, mo"tadil mintaqalarda urg'ochi hayvonlarning serpushtliligi shu asosda izohlanadi.

Populyatsiyadagi o'lim muayyan vaqt birligida nobud bo'lgan individlar soni bilan belgilanadi. Muhitning noqulay sharoitlari, yirtqichlar, parazitlar, kasallik va boshqa omillar individlar o'limiga sabab bo'ladi. Bir avlodga mansub individlarning to'liq yoshini yashagandan so'ng qisqa vaqt davomida nobud bo'lishi ekologiyada ideal holat sifatida qabul qilingan. Ushbu holatni egri chiziq sifatida ifodalanganda, u dastlab gorizontal o'qqa nisbatan parallel ketib, keyin pastga tomon keskin egiladi. Uni kuchli qabariq hayotchanlik chizig'i deyiladi. Tabiatda bunday hayotchanlik kam uchraydi, faqatgina ayrim qo'ng'izlarda, bolalarning yashovchanligi juda yuqori bo'lgan sut emizuvchilarda kuzatilishi mumkin. Laboratoriya sharoitida saqlanadigan meva pashshalarining hayotchanligi ancha yuqori va shu holatga yaqin bo'ladi.

Tabiatda ayrim hayvonlar hayotining dastlabki davrida ko'plab nobud bo'ladi. Ular uchun hayotchanlik egri chizig'i xos. Ushbu holatda hayotchanlikning egri chizig'i rivojlanishning dastlabki bosqichlaridayoq gorizontal o'q bo'yicha pasayib, so'ng vertikal yo'nalishda davom etadi. Baliqlarning uvildiriqlari nihoyatda mo'l bo'lib, ayrim hollarda suv yuzasini butunlay qoplab oladi. Uvildiriqlarning ko'pchiligi yirtqichlarga em bo'ladi yoki rivojlanmaydi. Tirik qolgan uvildiriqlardan voyaga etgan chavaqlarning taqdiri ham deyarli shunday kechadi. Oxir oqibatda, juda oz sondagi baliqlargina yashab qoladi. Ko'pchilik umurtqasizlar, amfibiyalar, qushlar va mayda kemiruvchilarning hayotchanligida shu holat kuzatiladi. Qadimgi odamlar - arxontroplar qoldiq suyaklarining deyarli yarmini 5 yoshdan 18 yoshgacha bo'lganlari tashkil etadi. Tabiatda hayotchanlikning uchinchi ko'rinishi, ya'ni oraliq hayotchanlik egri chizig'i ham uchraydi. Bunda organizmlarning miqdori, ularning hayoti davomida bir tekisda pasayib boradi. Bu holat akvariumda saqlanayotgan gidralarda qayd etilgan. 


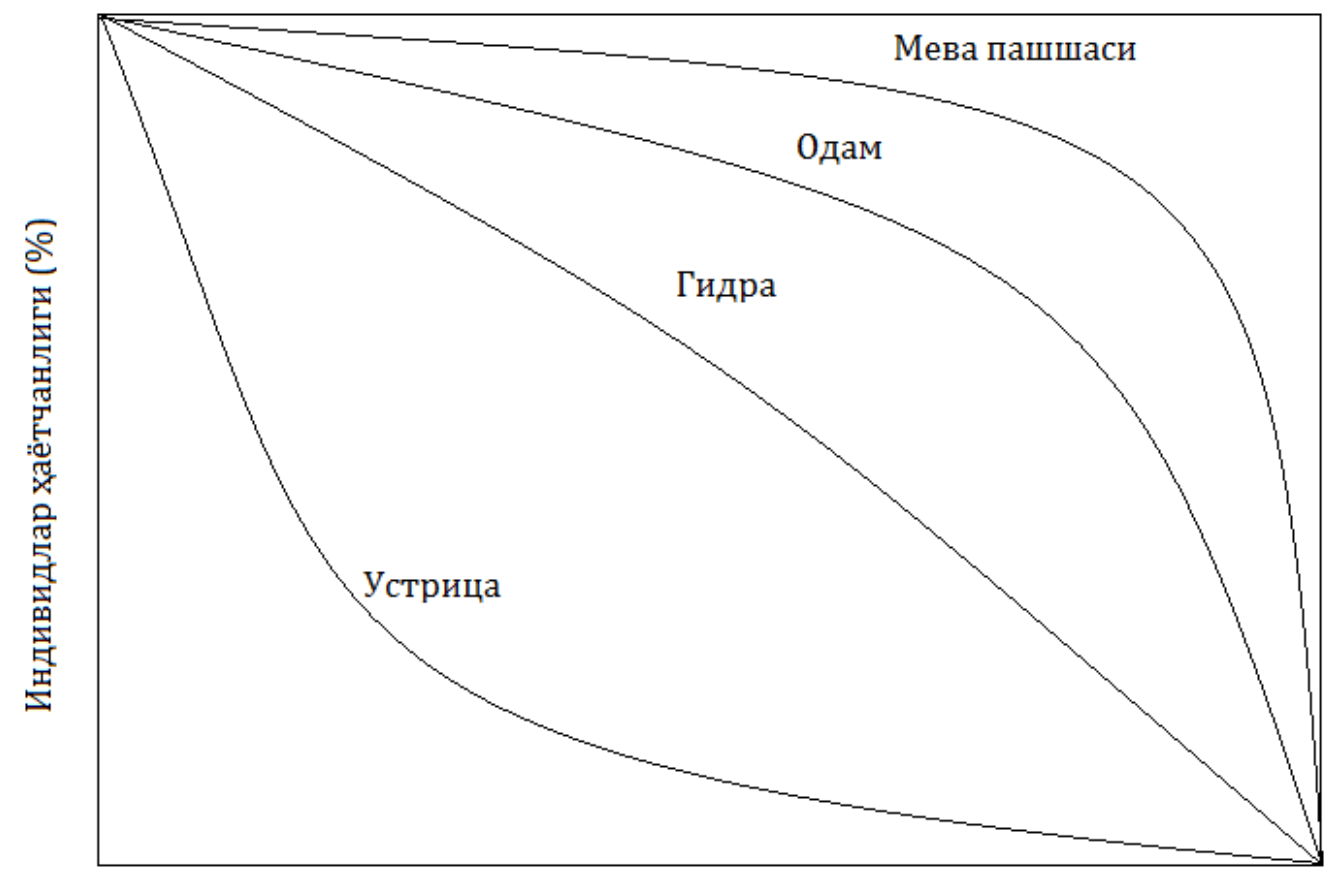

Авлодлар хаётининг давомийлиги

16-rasm. Hayotchanlik egri chiziqlarining har xil tiplari

Populyatsiyadagi individlarning bir qismini boshqa populyatsiyaga kelib qo'shilish ham miqdor zichlikka katta ta'sir etadi. Emmigratsiya natijasida populyatsiyalar o'rtasida individlarning almashinishi ro'y beradi. Organizmlar hayotining muayyan bir davrida o'z yashash joylarini o'zgartiradi. Emmigratsiya ko'pincha populyatsiyadagi yosh individlarning ulg'ayishi va tarqalishida ro'y beradi. Populyatsiyadagi o'lim va tug'ilish o'rtasidagi mutanosiblik uzilgan taqdirda faol siljishlar sodir bo'ladi. Natijada bir populyatsiyadagi ortiqcha individlar miqdor zichligi past bo'lgan populyatsiyalarga o'tib uni to'ldiradi. Bu holat populyatsiyadagi disspersiya deb nomlanadi. Tug'ilish, o'lim va emmigratsiyalar populyatsiyada turli darajadagi mavsumiy o'zgarishlarga sabab bo'ladi. Populyatsiya miqdor zichligiga ta'sir etuvchi omillar faoliyatini yashil olma shirasi misolida ko'rish mumkin.

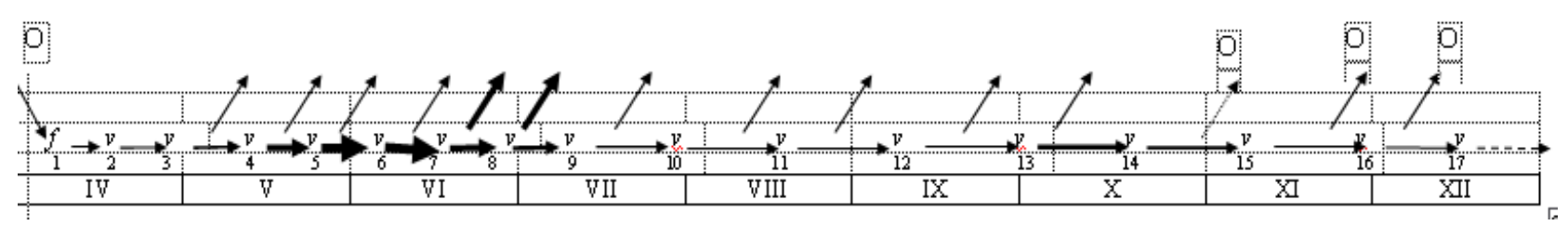

17-rasm. Yashil olma shirasining hayotiy tsikli

Rasmda ifodalangan yo'nalish konturining qalinligi omilning ta'sir 
kuchini ham belgilaydi. Populyatsiya miqdor zichligiga ta'sir etuvchi asosiy omillar - tug'ilish, o'lim va migratsiya darajasidir. Yashil olma shirasining serpushtliligi ovqat resurslariga bog'liq. Jumladan, bahor va dastlabki yoz oylarida daraxtlardagi yosh, sersuv barglarning bo'lishi ko'payish darajasining yuqori bo'lishiga sharoit yaratadi. Populyatsiyada miqdor zichlikning ortishi ozuqa resurslarining nisbiy kamayishiga mutanosib ravishda bog'liq bo'ladi. Muhitning abiotik omillari populyatsiya zichligiga ozuqa orqali va to'g'ridanto'g'ri ta'sir etadi. Jumladan, yoz oylarida haroratning ko'tarilishi va nisbiy namlikning kamayishi bilan olma barglari qalinlashib, dag'allashadi va shiralar oziqlanishi uchun noqulaylik tug'diradi. Natijada shiralar namlik nisbatan yuqoriroq bo'lgan joylar, yoki o'sayotgan olma daraxtlarining bachkilariga uchib o'tadi va hayotni davom ettiradi. Shiralarning qishlovchi tuxumlariga abiotik omillar to'g'ridan-to'g'ri ta'sir etishi mumkin. Masalan, qish iliq kelgan yillari yashil olma shirasi tuxumlaridan 90-95 \% sirkalar chiqishligi kuzatilgan, aksincha, qish fasli noqulay kelganda, ushbu ko'rsatkich $65-70 \%$ dan ortmaganligi qayd etilgan. Yashil olma shirasining mavsumiy ko'payishi kuzatilsa, aprel oyining o'rtalarida uning populyatsiya miqdor zichligi eng yuqori darajaga etadi. Bu davrda tur ichidagi munosabatlar etakchi ahamiyatga ega bo'ladi va boshqaruvchi omil sifatida populyatsiyada nimfa va qanotli shiralarning voyaga etishini tezlashtiradi.

Populyatsiya zichligini boshqarishda ozuqa resurslari cheklangan holda ro'y beradi. Shiralardan boshqa ayrim turlarda populyatsiya zichligining ortishini chegaralovchi mexanizmlardan biri o'zaro kimyoviy ta'sir etishdir.

Rivojlanayotgan itbaliqlar miqdor zichligi yuqorilay borishi jarayonida yirik itbaliqlar ajratgan maxsus modda mayda individlar rivojlanishini to'xtatib qo'yadi. Bitta etuk itbaliq 75 l suvda oziqlanayotgan barcha itbaliqlarning o'sishini cheklab qo'yishi mumkin. Yirik itbaliqlar voyaga etib, quruqlikka chiqib ketgach, suvda ular ajratgan kimyoviy moddaning ta'sir kuchi susayadi, shundan so'ng mayda itbaliqlar o'sishi davom etadi. Buning ekologik mohiyati shundan iboratki, suv havzasidagi barcha itbaliqlar bir muddatda voyaga etmaydi, ularda ketma-ket rivojlanish ro'y beradi. Dastlab etuk itbaliqlar oziq resurslarini jadal o'zlashtirish hisobiga metamorfozni tez tugallaydi. Ulardan so'ng mayda itbaliqlar uchun qulay sharoit yaratiladi.

Ozuqa zahirasining kamayish xavfi tug'ilganda, hayvonlarda o'z bolasini 
tutib eyish (kannibalizm hodisasi) kuzatiladi. Masalan, noqulay sharoitda olabug'a balig'i chavaqlarini eb hayot kechiradi. Davomli ocharchilik davrida yirik sut emizuvchilarda ham shunga o'xshash holat yuz beradi.

Yashil olma shirasi koloniyalarida qanotlilar ko'plab uchragan davrda nimfa va qanotlilar populyatsiyaning deyarli $2 G^{\prime} 3$ qismini tashkil etadi. Shu muddatda qanotli shiralarning migratsiyasi ro'y beradi va populyatsiya miqdor zichligi sekin-asta pasayib boradi. Populyatsiyada nobud bo'lgan individlar ham kuzatiladi.

Ma'lumki, yuqori miqdor zichlikdagi populyatsiyalarda turli kasalliklar tez tarqaladi. Foydali hasharotlar shiralar koloniyalariga aprel oxirlaridagina uchib o'tadi. Dastlab parazit parda qanotlilar - afidiidlar faol bo'lsa, keyinchalik shiralar miqdor zichligining boshqarilishida turli entomofaglar (xonqizi qo'ng'izi, oltinko'z, ularning qurtlari va boshqalar) faoliyati samarali bo'ladi. Entomofaglar yoz davomida shiralar sonini kamaytirib boshqarib turishda muhim ahamiyatga ega. Yuqoridagilar asosida shuni izohlash mumkin, ya'ni boshqaruvchi omillarning faollik darajasi populyatsiya miqdor zichligiga bog'liq. Populyatsiya zichligi eng yuqori darajaga etganda tur ichidagi munosabatlar asosiy ahamiyatga ega bo'lsa, faollikning quyi darajasi parazit va yirqich hasharotlarga bog'liq bo'ladi. Entomofaglar tabiiy tenglikni uzoq vaqt davomida bir me'yorda ushlab turishi mumkin bo'lgan boshqaruvchi omillardir.

Tabiatda bu omillar boshqa turlar populyatsiya zichligining boshqarilishida ham yashil olma shirasiniki kabi ketma-ket, bosqichmabosqich namoyon bo'ladi. 


\section{4-BO'LIM. BIOTSENOZ, BIOGEOTSENOZ VA EKOTIZIMLAR}

\section{Biotsenoz va undagi munosabatlar}

Ma'lum hududdagi barcha tirik organizmlar birligi bitsenozni hosil qiladi. Biotsenoz (lotincha "bios" - hayot, "tsenoz" - umumiy) bir xil ekologik sharoitga moslashgan va bir joyda yashaydigan barcha organizmlar birligidir. Chunki, tabiatda har bir tur yakka holda yashay olmaydi, balki atrofidagi boshqa tirik organizmlar bilan birgalikda hayot kechiradi. Biotsenozdagi organizmlar o'zaro bog'langan bo'lib, ular o'rtasidagi barcha munosabatlar uyg'unligi turlarning muhit omillariga bo'lgan talab darajasini ifodalaydi. Biotsenozning katta-kichikligi turlicha bo'lishi mumkin. Bunga g'o'za va boshqa madaniy o'simliklar biotsenozlaridan tortib, to tog' archazorlari, dasht va cho'l landshaftlaridagi barcha ko'rinishdagi biotsenozlar misol bo'la oladi.

Tirik organizmlarning tabiiy birliklari uchun o'ziga xos shakllanish, funktsiyalanish va rivojlanish qonuniyatlari amal qiladi.

Nemis ekologi V.Tishler ta'rifiga ko'ra, biotsenozlarning o'ziga xos xususiyatlari quyidagilarda namoyon bo'ladi.

Jamoalar doimo atrofdagi tarkibiy birliklar (turli turga yoki turlar tizimiga mansub organizmlar) hisobiga shakllanadi. Jamoalarning $\mathrm{u}$ yoki bu qismi almashib turadi. Jamoada alohida turlar yoki bir necha turlar o'rnini ekologik talab darajasi mutanosib bo'lgan boshqa turlar egallashi mumkin. $\mathrm{Bu}$ jarayon biotsenoz hayoti va uning yaxlitligiga ortiqcha ta'sir ko'rsatmaydi.

Organizm uyg'unlashgan hayotiy funktsiyalar hisobiga yaxlitligini saqlaydi, biotsenozlar barqarorligini qarama-qarshi munosabatlar uyg'unligi ta'minlaydi. Jamoalar bir turning miqdor zichligi ikkinchi tur tomonidan boshqariladi.

Biotsenoz katta tashqi muhit omillariga bevosita bog'liq bo'ladi. Abiotik muhitning biotsenoz egallagan o'rni bitop deb nomlanadi. Bitop (lotincha "bios"- hayot, "topos" - joy) - biotsenozning yashash va oziqlanish joyi.

Biotik munosabatlar majmui (yirtqich-o'lja, parazit-xo'jayin, kommensalizm, mutalizm va boshqalar) bitsenoz hayotining funktsiyalanishi va rivojlanishini ta'minlaydi.

V.N.Beklemishevning ta'rifiga muvofiq, biotsenozda 4 xil munosabatlar 
kuzatiladi: a) trofik, b) topik, v) forik, g) fabrik aloqalar.

Trofik aloqalar - biotsenozdagi eng muhim munosabatlar, ya'ni bir turni ikkinchi tur bilan yoki uning qoldig'i bilan oziqlanganda yuzalanadi. Biotsenozdagi parazit-xo'jayin va yirtqich-o'lja munosabatlari trofik aloqalar bir turning faoliyati natijasida ikkinchi tur uchun zarur bo'lgan oziqlanish sharoitidagi o'zgarishlar bilan ifodalanadi.

Tabiatda bir tur ikkinchi hayvon uchun yashash sharoitini yaratib beradi. Ektoparazitlar, go'ngda hayot kechirayotgan hasharotlar topik aloqalar asosida yashaydi va rivojlanadi. Biotsenozda trofik va topik aloqalar muhim o'rin tutadi va uning hayoti va funktsiyalanishini ta'minlovchi omillardan sanaladi.

Forik aloqalar bu bir turni boshqa tur yordamida tarqalishida kuzatiladi. Tabiatda o'simlik urug'lari, mayda hayvonlar boshqa organizmlar vositasida bir muncha uzoq masofalar bo'ylab tarqaladi. Ko'pchilik bo'g'imoyoqlilar, qushlar va sut emizuvchilar tanasida, yung va patlari orasida bir mintaqadan ikkinchisiga ko'chishi mumkin.

Fabrik aloqalari tufayli bir hayvon uya qurishi uchun ikkinchi hayvon yoki o'simlikdan, ularning turli qoldiqlaridan foydalana oladi. Qushlar uya qurish uchun sut emizuvchilar yunglaridan, boshqa qushlarning patlari va o'simlik qoldiqlaridan foydalanadi. Buloqchilar uyalari o'simlik qoldiqlari, yumshoqtanlilarning chig'anoqlari bo'laklaridan tiklanadi.

Biotsenoz tuzilmasi. Biotsenozlar organizmlarning tasodifiy yig'ini emas, u hayot shakllanishining o'ziga xos xususiyatlaridan biri sifatida aniq tuzilmaga ega. Biotsenozning tur tarkibi, fazodagi-kenglikdagi va ekologik tuzilmalari farqlanadi.

Biotsenozning tur tarkibi. Turlar xilma-xilligi, ular o'rtasidagi miqdoriy nisbatlar biotsenozning tur tarkibini tashkil etadi. Tabiatda turlarga boy bo'lgan va kambag'al biotsenozlar uchraydi. Muhit sharoitlari o'ta noqulay bo'lgan mintaqalar (shimoliy kengliklar, cho'l va yuqori tog' mintaqalari, okeanlar tubi va boshqalar) biotsenozlarning turlar xilma-xilligi past darajada bo'ladi. Ekologik sharoit qulay bo'lgan tropik va tog' o'rmonlari, daryo vohalari biotsenozlari turlarga boyligi bilan xarakterlanadi.

Tabiiy biotsenozlarning tur tarkibi madaniy biotsenozlarnikiga nisbatan barqoror va xilma-xil bo'ladi. Inson faoliyatining mahsuli bo'lgan madaniy 
biotsenozlar - agrotsenozlar deb nomlanadi. Agrotsenozlardagi turlar xilmaxilligi, miqdoriy ko'rsatkichlari uning tarixiga, yoshiga ham bog'liq. Yangi shakllanayotgan yosh biotsenozlarda kam sondagi turlar uchraydi, vaqt o'tishi bilan undagi turlarning sifat va miqdor tarkibi borqarorlashib boradi. Shuni e'tiborga olish zarurki, eng sodda biotsenozda ham kamida bir necha o'n tur uchraydi. Bitsenozdagi turlar xilma-xilligini ortishida o'simliklar dunyosi asosiy ahamiyatga ega bo'ladi. Odatda qalin qoplamli hayvonlar va mikroorganizmlar uchun xilma-xil yashash joylarini shakllanishiga olib keladi.

Yashash sharoitlarining xilma-xilligi biotsenozning turlarga boy bo'lishligini ta'minlaydi. Biotsenozda har bir organizm qulay sharoit topishi bilan birga, u bilan trofik va topik aloqada bo'lgan boshqa organizmlarning yashashi uchun zarur bo'lgan sharoitni yuzaga kelishiga sabab bo'ladi.

Biotsenozdagi turlar soni va xilma-xilligini o'rganishda dastlab ularning ro'yxati tuziladi va qiyosiy tahlil etiladi. Lekin bu holat taksonomik jihatdan bir muncha noqulaylikka sabab bo'ladi. Ma'lum hududni egallovchi biotsenozni to'lig'icha tadqiq etish ancha mushkul ish, shuning uchun uning turli joylaridan namunalar olish maqsadga muvofiq. Shuni ta'kidlash lozimki, olinayotgan namunalarga, eng avvalo, mazkur biotsenozda eng ko'p tarqalgan turlar to'g'ri keladi. Undagi barcha turlar haqida ma'lumot to'liq bo'lish uchun namunalar soni ko'p bo'lishi zarur. Lekin namunalar soni ma'lum darajada cheklangan bo'lishi kerak. Masalan, biotsenozda 5 turga mansub 100 individ yashasa, ulardan 96 tasi bir turga qolgan 4 tasi alohida turlarga mansub bo'lishi mumkin. Bu holda mazkur biotsenoz bir turdangina iborat bo'lganday tasavvur etiladi. Aksincha holat ham bo'lishi mumkin, ya'ni har bir tur 20 tadan individga ega bo'lib, jami 100 donadan tashkil etishi mumkin, bunday holatga biotsenoz boshqacharoq e'tirof etiladi. Ayni yo'nalishdagi qiyinchiliklarning oldini olish uchun xilma-xil ko'rsatkichdan faydalaniladi. Biotsenozdagi turlar xilma-xilligi Simpson va Shennot ko'rsatkichlari asosida tahlil etiladi.

Simpson ko'rsatkichi quydagi formula asosida ifodalanadi:

1 (D)=SYPi2I=1 bunda, 1-turning ayni jamoadagi ulushi Pi bo'lsa, S mazkur jamoadagi barcha turlar sonini ifodalaydi.

Shennon ko'rsatkichi quydagicha ifodalanadi: $\mathrm{H}=-\sum \mathrm{Pi} 2 \log 2 \mathrm{Pi}$

Ushbu tenglamada $\sum$ - summa, Pi har bir turning mazkur biotsenozdagi 
ulushi (miqdori yoki zichligi massasi), log2Pi esa Pi qiymatining ikkilama logarifmi.

Son jihatdan ko'p bo'lgan va ko'zga yaqqol tashlanadigan tur ayni biotsenozda dominant (hukmron)lik qiladi va uni dominant tur deb nomlanadi. U bitsenozning yadrosini tashkil etadi. Dominant turlarning barchasi ham biotsenozga o'z ta'sirini bir xil darajada o'tkazmaydi. Ularni orasida maxsus muhitni hosil qiluvchi turlar - edifikatorlar ajralib taradi. Edifikatorlar biotsenozning «quruvchi» lari bo'lib uning xususiyatlarini belgilab beradi.

Biotsenozdagi har bir turning o'rnini aniqlashda uning mo'lligi e'tiborga olinadi. Bu ko'rsatkich odatda muayyan hududga nisbatan ifodalanadi. Masalan, tarqalish darajasini belgilaydi. Tarqalish darajasi biotsenozda turlarning uchrash chastotasini ifoda etadi. U umumiy namuna maydonchalar sonini tur uchragan maydonchalar soniga qiyoslash asosida aniqlanadi. Dominantlik darajasi ma'lum bir tur miqdor zichligining, ushbu guruhdagi umumiy zichlikka bo'lgan nisbatini bildiradi. Masalan, ma'lum hududda uchrovchi 2000 ta qushning 80 tasini maynalar tashkil etsa, uning dominantlik darajasi $40 \%$ ni tashkil etadi. Shunday qilib, biotsenozlarning sifat va miqdor ko'rsatkichlarini tahlil etish asosida uning o'ziga xos xususiyatlari va barqarorligi haqida ma'lum xulosaga kelish mumkin.

Biotsenozdagi topik aloqalar zamirida konsortsiyalar tashkillanadi.

Konsortsiya - ma'lum tur tanasiga joylashib boshpana va ozuqa topgan turlarning o'ziga xos ekologik birligi. Biotsenozdagi har bir tur atrofida o'ziga xos yashash sharoitini vujudga keltiradi. Natijada u konsortsiyaning markaziy turi sifatida boshqa turlarni trofik va topik aloqalar asosida o'z atrofida mujassamlantiradi. V.N.Beklemishev ta'riflashicha, dala sichqonlari ektoparazitlari, ichki organlardagi murakkab konsortsiyadir. Biotsenozda yirik konsortsiyalar bilan bir qatorda, bir qancha mayda - birinchi, ikkinchi, uchinchi tartibdagi konsortsiyalar ham farqlanadi. Umuman, biotsenoz birbiri bilan topik va trofik aloqalar asosida mustahkam bog'langan konsortsiyalar tizimidir.

Biotsenozning fazoviy tuzilmasi. Barcha biotsenozlarda o'simliklar asosiy o'rinni egallaydi. Hayvonlar o'simlik bilan ozuqa zanjiri orqali bog'langan. Hayvonlar daraxt va butalarning turli qismlarida ma'lum yaruslar 
(qavatlari) bo'ylab tarqaladi. O'simlik jamoalaridagi har bir qavat o'zining mikroiqlimiga ega bo'lib, o'ziga xos yorug'lik, namlik, harorat va boshqa ekologik sharoitlari bilan farqlanadi. Ko'pchilik hayvonlarning hayoti biotsenozdagi ma'lum qavat bilan mustahkam bog'langan. Masalan, hasharotlar orasida tuproqda yashovchilar - geobiylar, tuproq yuzasida yashovchilar - gerpetobiylar, mox to'shamalarida uchrovchilar - bribiylar, o'tsimon o'simliklardagi - fillobiylar va nisbatan yuqori yaruslarda yashovchi - aerobiylar farqlanadi.

Qushlar o'rtasida tuproq yuzasida (tovuqsimonlar, qurlar, yo'rg'a to'rg'aylar, dehqon chumchuqlar), butalar va daraxtlarga (qorayaloqlar, moyqutlar, sayhalar, bulbullar va boshqalar) daraxt tanasidagi qovoqlarga (qirg'iylar, panqushlar) uya qurib bola ochadi.

Biotsenozning ekologik tuzilmasi. Har bir biotsenozda alohida ekologik guruhga mansub organizmlar uchraydi. Biotsenozdagi ekologik guruhlar nisbati uning ekologik tuzilmasini ifodalaydi. O'xshash ekologik tuzilmaga ega bo'lgan biotsenozlarning har birida o'ziga xos tur tarkibi bo'lishi mumkin. Chunki qiyoslanayotgan biotsenozlardagi mutanosib ekologik tokchani muhit omillariga bo'lgan talab darajasi bir muncha yaqin boshqa-boshqa turlar egallashi mumkin. Filogenetik uzoq, lekin ekologik talab darajasi mutanosib bo'lgan va biotsenozlarda bir xil funktsiyani bajaruvchi turlar vikar turlar deb ataladi. Tabiatda ekologik vikarlar keng tarqalgan. Masalan, Evropa o'rmonlarida suvsarlar, Osiyo o'rmonlarida sobollar, bir xil ekologik tokchani egallab hayot kechiradi. Afrika kengliklarida antilopalar, Shimoliy Amerika bizonlari, Osiyoda yashovchi yovvoyi otlar va qulonlar o'xshash ekologik tokchalar bo'ylab tarqalgan va tegishli biotsenozlarning ozuqa zanjirida o'rni va ahamiyati o'xshashdir.

Har bir biotsenozning tur tarkibi mazkur mintaqada tarqalgan turlardan tashkillanadi. Lekin biotsenozlarning ekologik tuzilmasi ma'lum iqlim va landshaft sharoitiga bog'liq holda shakllanadi. Turli mintaqalar bo'ylab tarqalgan o'xshash biotsenozlarda fitofaglar va saprofaglar nisbati qonuniy o'zgaradi. Biotsenozlarning ekologik tuzilmasi ulardagi gigrofil, mezofil va kserofil hayvonlar ulushini ham ifodalaydi. Biotsenozda u yoki bu ekologik guruh vakillarining ustunligi ayni hududdagi ekologik sharoitni uning fizikkimyoviy xususiyatlarini tahlil etish imkoniyatini beradi. 
G'o'za agrotsenozi va uning tuzilmasi. Agrotsenozlar - bu ikkilamchi biogeotsenozlardir. Ularning vujudga kelishi, shakllanishi va tiklanishi bevosita inson faoliyati bilan bog'liq. Shuning uchun agrotsenozlarni boshqacha tarzda madaniy biotsenozlar deb ham aytiladi. Agrotsenozlar mavjud maydondagi ekin turiga qarab nomlanadi: g'o'za agrotsenozi, yo'ng'ichka agrotsenozi, poliz ekinlari agrotsenozi, bog' agrotsenozi va boshqalar.

Agrotsenozlar barchasi tabiiy bitsenozlar o'rnida vujudga keladi. Lekin, agrotsenozlarda ham turli turlarga mansub organizmlar tevarak atrofdagi jonsiz tabiat sharoitiga evolyutsion jarayonda shakllangan tabiiy qonuniyatlar asosida bog'langan bo'ladi. Har qanday agrotsenoz ham tirik organizmlarni noorganik tabiat bilan mustahkam kompleks bo'lib, o'zini saqlab turadi va bu sistemada moddalar davra bo'ylab aylanib yuradi. Bu holatni g'o'za agrotsenozi misolida ifodalab berish mumkin.

G'o'za agrotsenozi asosini - produtsent hisobiga g'o'za o'simligining o'zi tashkil etadi. Uning hisobiga birlamchi konsumentlar - o'simlikxo'r organizmlar yashaydi, jumladan, shiralar, o'rgimchakkana, ko'sak qurti, ayrim qandalalar va boshqalar. Umuman, g'o'zada 30 ga yaqin zararkunanda uchraydi, ulardan faqat 5-6 tasigina bu o'simlikka doimiy zarar keltirib yashaydi. Lekin bularning hammasi ham g'o'zaga bir xil zarar etkazmaydi. Erta bahorda yangi ungan g'o'za maysalari asosini tomir qurti kemiradi. Keyinchalik g'o'zaning chin barglarida shiralarni uchratish mumkin. Ular astasekin ko'payib g'o'za barglarini bujamaytirib qo'yishi mumkin. Bu bo'g'imoyoqlilarning ko'payishi ular hisobiga yashovchi organizmlar, ikkilamchi konsumentlar, yirtqich va parazit hasharotlari g'o'za agrotsenozida yig'ilishiga sabab bo'ladi. Bular xonqizi qo'ng'izlari, oltinko'zlar, parazit pardaqanotlilar va ayrim foydali qandalalardir. Bu hasharotlar g'o'zada yashovchi zararli turlar sonini kamaytirib, boshqarib turishda muhim rol o'ynaydi. Masalan, etti nuqtali xonqizi bir kunda 100-130 ta, oltinko'z lichinkasi 60-70 ta shirani eya oladi. Sirfid pashshasi esa bir kunda 200 dan ortiq shiraga qiron keltiradi. Lekin, bu foydali hasharotlar o'z navbatida uchlamchi konsumentlar - o'ta parazit hasharotlar (ayrim pardaqanotlilar)ga ozuqa bo'lib xizmat qiladi. Shunday qilib, g'o'za agrotsenozida ham emanzor agrotsenozidagi kabi 4-5 zvenodan tuzilgan ozuqa zanjiri kuzatiladi. 
G'o'za agrotsenozidagi konsumentlar (ayniqsa, yirtqich va parazit hasharotlar) o'rtasida ovqat orqali raqobat kuzatiladi. Jumladan, yirtqich qandalalar ko'p bo'lsa, ko'sak qurtini parazit hasharotlar bilan zararlanganlari kamroq uchraydi. Chunki qandalalar g'o'za tunlami va kichik yoshdagi ko'sak qurti bilan oziqlanadi, parazitlarda qanotlilar - ixnevmonid va brakanoidlar esa katta yoshdagi qurtlarning tanasida voyaga etadi. Agar oltinko'z, xonqizi qo'ng'izlari va sirfid pashshalari lichinkalari soni ko'p bo'lsa, afidiid - parda qanotlilar bilan zararlangan shiralarni ham qiradi.

Paxta dalalarida yuqoridagilardan tashqari o'simlik qoldiqlari, nektar, shiralar ajratgan suyuqlik bilan ovqatlanadigan, shuningdek, yovvoyi o'simliklarda yashovchi hasharotlar (chumolilar, arilar, ayrim qo'ng'izlar, o'rgimchaklar va boshqalar) uchraydi. Bu bevosita g'o'za o'simligi bilan bog'lanmagan bo'lsa-da, ayni agrotsenozning ajralmas qismi hisoblanadi va ayni ekosistemadagi tabiiy tenglikni tartibga solib turishda muhim rol o'ynaydi.

G'o'za agrotsenozidagi barcha tirik organizmlarni qoldiq va chiqindilari paxta dalalariga tushadi. Ular redutsentlar - turli bakteriyalar, saprofit zamburug'lar, ayrim umurtqasiz hayvonlar yordamida parchalanadi va mineral moddalarga aylanadi. Redutsentlar soni tuproqda nihoyatda yuqori darajada bo'ladi. Masalan, $1 \mathrm{~m}^{2}$ paxta dalasida faqat sodda hayvonlarnigina 100 mln donadan ortig'i uchraydi.

Yuqoridagilardan ko'rinadiki, barcha tabiiy biogeotsenozlardagi kabi g'o'za agrotsenozida ham produtsent, konsument va redutsent tizimlari orqali murakkab ozuqa zanjiri mavjud bo'ladi. Olimlarning aniqlashicha, O'zbekiston paxta dalalarida 300 dan ortiq (tuproqdagi organizmdan tashqari) umurtqasiz hayvonlar uchrar ekan. Tabiiy ekosistemalardagi kabi g'o'za agrotsenozida ham turlar soni va ularning xilma-xilligi o'zgarib turadi. Bunda g'o'zaning ilk vegetatsiya davrida har 100 tup o'simlikda 25-30 dona foydali hasharot kuzatilsa, yoz o'rtalariga kelib, bu son deyarli 10-12 barobar ortadi. Ayniqsa, yozning ikkinchi davrida 100 o'simlikda 360-450 tagacha foydali hasharotni kuzatish mumkin. Qish iliq kelgan yillari tomir qurti yaxshi qishlaydi va ko'plab saqlanib qoladi, natijada, bahorda g'o'za nihollariga kuchli zarar etkazadi. Shunday holat Farg'ona vodiysida 1981 yilning bahorida kuzatilgan. Odatdagi yillarda paxta dalalarining $1 \mathrm{~m}^{2}$ maydonida 1-2 tomir qurti 
kuzatilgan bo'lsa, o'sha yili zararkunanda soni $1 \mathrm{~m}^{2}$ da 17-20 taga etgan. Ilmiy ma'lumotlarda ko'rsatilishicha, bu holat keyingi 15 yil davomida kuzatilmagan.

G'o'zaning boshqa zararkunandasi o'rgimchakkana yoz issiq va quruq kelganda ko'payishi mumkin. Agrotsenozlarda turlar sonining o'zgarishida inson faoliyati ham katta rol o'ynaydi. Masalan, zararli hasharotlarga qarshi tartibsiz va yoppasiga kimyoviy zaharlarni qo'llanishi ularni nobud qilsa-da, birinchi navbatda, foydalilarni ko'plab qirilishiga sabab bo'ladi. Zaharlardan omon qolgan ma'lum sondagi zararkunandalarni esa ximikatlarga chidamliligi ortib, zo'r berib ko'payadi va g'o'zaga jiddiy zarar etkazadi. Shuning uchun ham agrotsenozdagi har bir turni bioekologiyasini o'rganish undagi o'zgarishlarni hisobga olgan holda kimyoviy vositalardan oqilona foydalanish lozim. Bu esa ekologiyaning eng muhim vazifalaridan biridir.

\section{Biotsenozlar va ekotizimlar}

Biotsenozdagi barcha organizmlar noorganik muhit bilan bog'langan. O'simliklar karbonat angidrid, kislorod, suv va tuproqdagi mineral tuzlarni o'zlashtiradi. Geterotroflar ozuqa zanjiri orqali avtotroflar bilan bog'langan. Organizmlar uchun ular yashayotgan hududdagi noorganik moddalar jadal o'zlashtirilgan taqdirda, bu zahira qisqa muddat qoldiqlari bilan ushbu zaxirani yangilab, boyitib boradi. Hayvonlar havodagi kislorodni o'zlashtirib, karbonat angidridni ajratib chiqaradi. Ularning chiqitlari (siydik, tezaklari) tuproqqa tushadi.

Hayvonlarning jasadlari va o'simlik shox-shabbalarining chirib parchalanishi bilan tuproqdagi biogen moddalar ko'payadi. Shunday qilib organizmlar bilan noorganik muhit o'rtasida o'ziga xos aloqa tizimi amalga oshadi. Organizmlarni noorganik komponentlar bilan bo'lgan birligi asosida moddalarning davra bo'ylab aylanishi sodir bo'ladi. Moddalarning davra bo'ylab aylanishini ta'minlovchi organizmlar va noorganik komponentlar majmui ekotizim deyiladi. Ekotizim tushunchasi ilk marotaba ingliz ekologi A.Tensli (1935) tomonidan fanga kiritilgan. Tenslining talqiniga ko'ra ekotizimlarda abiotik va biotik omillar bir xil daraja doirasida bo'ladi va shu asosda ular o'rtasida mustahkam munosabatlar shakllanadi. Shuningdek, 
ekotizim tabiatning asosiy birligi sifatida uning hajmi chegaralangan emas va turli darajadagi kenglikni egallashi mumkin. Ekotizmlar kontseptsiyasi bilan bir davrda biogeotsenozlar haqidagi ta'limot ham yuzaga keldi. Bu ta'limot asoschisi akademik V.N.Sukachev (1942) edi. Ekotizim turli darajadagi kenglik bo'ylab moddalar aylanashini inobatga olsa, biogeotsenoz alohida olingan muayyan o'simlik qoplamiga, fitotsenozga xos tushuncha.

Biogeotsenologiya geobotanikaning bir tarmog'i sifatida shakllanib, u ma'lum sharoitda fitotsenozda tuproq, hayvonlar, mikroorganizmlar va boshqa omillar bilan birligini tadqiq etadi.

Ekotizim va biogeotsenoz kontseptsiyalari biri-birini to'ldiradi va biri ikkinchisi uchun manba bo'lib xizmat qiladi. Ekotizimlar biogeotsenozga nisbatan kenroq tushuncha hisoblanadi.

Ekotizimda anorganik moddalar zahirasi va uch xil ekologik guruhga mansub organizmlar bo'ladi. Birinchi guruhga yashil o'simliklar kiradi va produtsentlar deb nomlanadi. Produtsentlar barcha biotsenozlarning asosini tashkil etib, quyosh energiyasini bog'langan kimyoviy energiyaga aylantiradi, energiyaning bir qismi zahirada saqlanadi. Produtsentlarning ayni shu qismi ikkinchi guruh organizmlar - konsumentlar tomonidan o'zlashtiriladi. Hayvonlar o'simliklar tomonidan g'amlangan organik moddaning iste'molchilaridir. Ozuqa zanjiridagi o'rniga qarab, turli tartibli konsumentlar guruhi farqlanadi. Ikkinchi tartibli konsumentlar guruhiga o'txo'r hayvonlar bilan oziqlanuvchi yirtqichlar kiradi. Yirtqichlar tanasida parazitlik bilan hayot kechiruvchi sodda hayvonlar, chuvalchanglar va boshqalar uchinchi tartibli konsumentlar hisoblanadi.

O'simlik va hayvonlarning qoldiqlari, jasadlar o'zida energiya saqlaydi. Nobud bo'lgan o'simlik va hayvonlarning organik qoldiqlari mikroorganizmlar, ya'ni saprofit holda yashovchi bakteriyalar va zamburug'lar ta'sirida parchalanadi. Bu guruhga mansub organizmlar redutsentlar deb ataladi. Produtsentlar va konsumentlar qoldiqlari redutsentlar yordamida parchalanib produtsentlar o'zlashtira oladigan darajaga keladi. Ayni ekologik guruhlar o'rtasida mustahkam ozuqa zanjiri vujudga kelib, ular bo'ylab moddalar aylanishi amalga oshadi. 


\section{Biogeotsenozlar mahsuldorligi}

Biotsenozlar hayoti doimiy energiya oqimi va unda yig'ilayotgan organik modda miqdoriga bog'liq bo'ladi. Biogeotsenozda jamlanayotgan organik massa mahsuldorlikni ifodalaydi. Biogeotsenozlarda birlamchi va ikkilamchi mahsuldorlik farqlanadi. Produtsentlar karbonat angidrid, suv va quyosh energiyasi hisobiga birlamchi mahsuldorlikni (BM) sintezlaydi, konsumentlar va redutsentlar mahsuli ikkilamchi (IM) hisoblanadi. O'simliklarni vaqt birligida sintezlagan organik massasi yalpi birlamchi mahsuldorlikni (YaBM) tashkil etadi. Yalpi birlamchi mahsuldorlikning bir qismi o'simlikning nafas olishi uchun sarf bo'ladi. Masalan, tropik o'rmonlarda yalpi birlamchi mahsuldorlikning 40-70\% o'simliklarning nafas olishiga sarf etiladi. Yalpi birlamchi mahsuldorlikning o'simliklar o'z hayotiy jarayonlari uchun sarf etilganidan qolgan qismi sof birlamchi mahsuldorlik (SBM) deb ataladi.

Sof holdagi birlamchi mahsuldorlik konsument va redutsentlar uchun energiya zahirasi hisoblanadi. Konsumentlar tomonidan ikkilamchi mahsuldorlik to'planadi. Quruqlikning yillik birlamchi mahsuldorligi 110-120 mlrd t quruqlik massasini tashkil etadi. Dengizlardagi bu ko'rsatkich 50-60 mlrd. $t$ ni tashkil etadi. Okeanlar er sharining 2G'3 qismini tashkil etsada, er yuzasidagi mahsuldorlikni 1G'3 qismini beradi. Er sharining asosiy qismidagi mahsuldorlik $1 \mathrm{~m}^{2}$ ga $400 \mathrm{~g}$ ni tashkil etadi. Bu ko'rsatkich quruqlikning 30\% va okeanlarning $90 \%$ ga to'g'ri keladi.

Ochiq okeanlar quruqlikning cho'l mintaqalari bilan bir xil hisoblanadi va ulardagi mahsuldorlik o'ta past bo'ladi. Aksincha, botqoqliklar, qirg'oq mintaqalari, ishlov beriladigan erlar mahsuldorligi yuqori bo'ladi. O'rmon biomlarida ninabargli o'rmonlardan tropik o'rmonlarga tomon mahsuldorlik ortib boradi. Bu holat yorug'lik, namlik, harorat va boshqa omillarga bog'liq holda izohlanadi.

\section{Energiya oqimi}

Ekotizimlarning funktsiyalanishi uchun energiya fotosintez jarayoni hisobiga yig'iladi. Ekotizimlarni mustaqil organik massani yig'ishi avtoxton jarayon deb nomlanadi. Ekotizimlarga organik massa tashqaridan loyqa bilan, oqim orqali va shamol vositasida kelishi mumkin. Bu holat alloxton jarayon nomini olgan. Avtotroflar va geterotroflar o'rtasida shakllangan ekologik va 
evolyutsion bog'liqlik tufayli tabiatda biogen moddalar uzluksiz aylanib turadi. Organizmlar Quyosh energiyasini kimyoviy, mexanik va issiqlik energiyalariga aylantiradi. Mazkur jarayonni har bir bosqichida energiya sarfi kuzatiladi, quyi bosqichda issiqlikka aylanib tarqab ketadi. O'simliklar tomonidan hosil qilib jamlangan energiya biogeotsenozlarning ozuqa zanjiri bo'lib taqsimlanib boradi, ya'ni 4-6 bo'g'indan tashkil topgan ozuqa zanjiridagi organizmlar ketma-ketlikda bir-biri bilan oziqlanishidan tashqari, noqulay sharoitda boshqa ozuqa zanjiri tarkibidagi organizmlar bilan ham oziqlanishi mumkin. Bir qancha ozuqa zanjirlari o'zaro bog'lanib, bog'lanish to'rini hosil qiladi.

Konsumentlar o'rtasidagi modda va energiyaning o'zgarishi kuzatilganda ma'lum bo'ladi-ki, ular qabul qilgan ozuqa to'lig'icha xazm bo'lmaydi. Ozuqaning xazm bo'lmagan qismi ahlat bilan tashqariga chiqarib tashlanadi. Chiqindilar ozuqa zanjiridagi boshqa organizmlar uchun energiya manbai hisoblanadi.

Ozuqa o'zlashtirish ko'rsatkichi uning tarkibi va hayvonlarning ovqat xazm qilish tizimiga bog'liq bo'ladi. Masalan, saprofaglar ozuqani $10-12 \%$ ni, yirtqichlar - 75\% ini o'zlashtira oladi. Energiyaning asosiy qismi organizmning hayotiy jarayonlari uchun sarf bo'ladi, ma'lum qismi dissimilyatsiya mahsulotlarini tashqariga chiqarib tashlash uchun sarf bo'ladi. Organizmdagi metabolitik jarayonlarni ta'minlash uchun sarflanadigan energiya nafas olish energiya sarfi deb nomlanadi. Chunki organizmdan ajralayotgan karbonat angidrid miqdori asosida undagi energiya sarfini ifodalash mumkin. O'zlashtirilgan ozuqaning oz qismi organizmning o'sishi, zaxiraga energiyani to'plash va umumiy og'irligini oshirishga ketadi. Turli tartibli konsumentlardan yoki bir trofik darajadan ikkinchisiga o'tayotganda energiya va mahsulotlar kamayib boradi. Har bir trofik darajaning mahsuldorligi avvalgi daraja mahsuldorligi bilan chegaralanadi.

Trofik darajalari qolgan mahsulot va energiya zahiralari o'lik organik qoldiq bilan birga biotsenozning umumiy energiya va mahsulotlar zaxirasini tashkil etadi.

Ikki xil ozuqa zanjiri farqlanadi:

a) sarflanish zanjiri;

b) parchalanish zanjiri. 
Trofik zanjirlar avtotroflardan boshlansa, sarflanish zanjiri deyiladi.

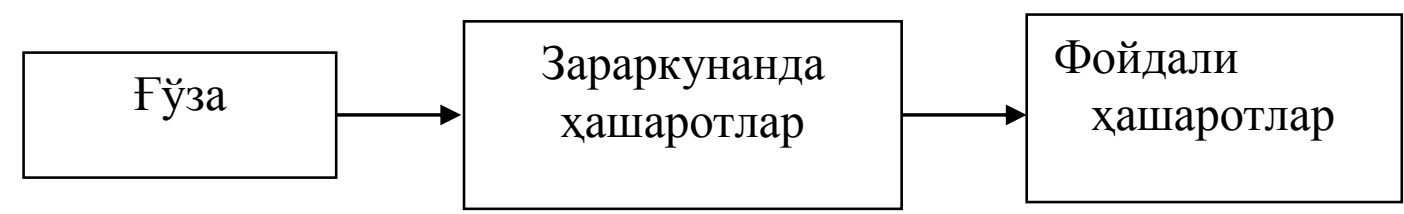

Agar ozuqa zanjiri o'simlik qodiqlari, hayvonlar jasadi va chiqindilaridan boshlansa, parchalanish (detrit) zanjiri deb ataladi.

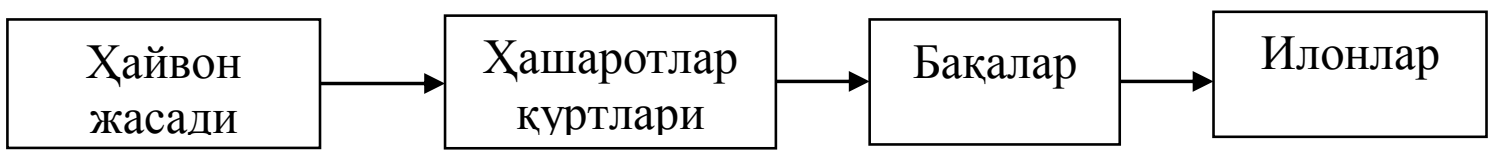

Ikkilamchi mahsuldorlik yangi biomassa hosil bo'lish jarayoni tezligini ifodalaydi. O'simliklardan farqliroq, hayvonlar oddiy molekulalardan murakkab mikromolekulalarni sintezlay olish imkoniyatiga ega emas. Ular uchun energiya manbai o'simliklar yoki boshqa geterotroflar hisoblanadi. Birlamchi va ikkilamchi mahsuldorlik o'rtasida bog'liqlik bo'lishi bilan birga, ma'lum mutanosiblik zarur. Masalan, ko'l zooplanktonlarning biomassasi fitoplanktonlar biomassasiga bog'liq, cho'l hayvonlarining mahsuldorligi o'to'lanlar mahsuldorligi bilan belgilanadi.

Trofik tuzilmada produtsentlar biomassasi konsumentlarnikidan doimo yuqori bo'ladi. Mahsuldorlik piramidalar shaklida ifodalanganda, keng asosni produtsentlar va keyingi darajalarni konsumentlar va redutsentlar tashkil etadi. Shunday qilib tabiatda ekologik piramida hosil bo'ladi.

Ekologik piramida ilk marta Ch.Elton (1927) tomonidan tuzilib sonlar piramidasi deb atalgan.

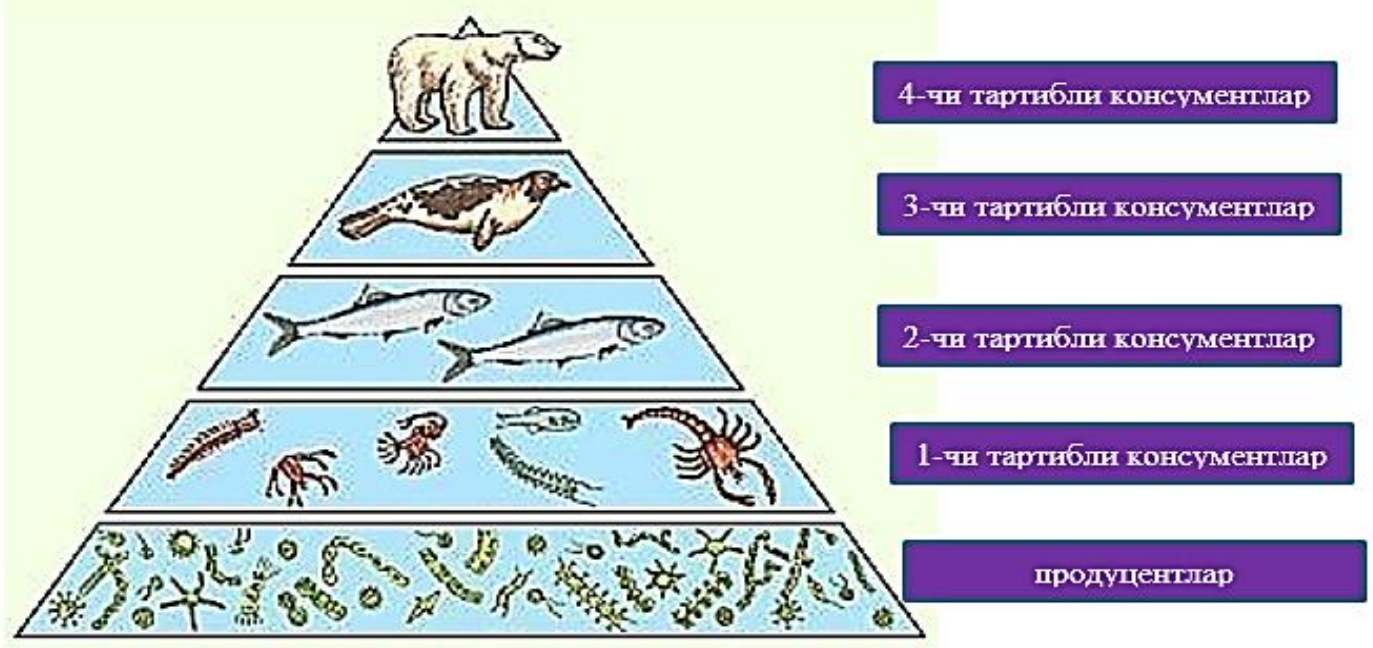

18-rasm. Biomassaning kamayib borish ekologik piramidasi 
Piramidalar sonlarda, biomassa va mahsulot birligida ifodalanadi. Quruqlik ekotizmlarida biomassa piramidalar qoidasi qo'llaniladi. Barcha ekotizimlarda birlamchi va ikkilamchi mahsuldorlikning nisbatlari, ya'ni mahsulotlar piramidalari ifodasi kuzatiladi. Tabiatda istisno tariqasida piramidalar qoidasidan biroz cheklanishlar ham kuzatiladi. Jumladan, quruq yog'ochlikda oziqlanayotgan qurtlarning miqdor zichligi o'simliknikiga nisbatan yuqori bo'ladi. Aksincha, qisqa muddatli hayot tsikliga va yuqori mahsuldorlikka ega bo'lmagan suv o'tlari, bir muncha uzoq umr ko'ruvchi mahsuldor zooplanktonlar biomassasini bir me'yorda ushlab turishi mumkin.

Jamoadagi energiya oqimi birlamchi mahsuldorlikni umurtqasiz fitofaglar yoki o'txo'r hayvonlar o'zlashtirishidan boshlanadi. Energiyaning bir qismi ularning nafas olishi uchun sarf bo'ladi. Ular bilan oziqlangan yirtqichlarning qoldiqlari va jasadi zamburug'lar tomonidan o'zlashtiriladi, bir qismi tuproq kanalari faoliyati nitijasida parchalanadi. Har bir bosqichda o'zlashtirib ulgurmagan qoldiq energiya manbai sifatida zahiralanadi. Ozuqa zanjirining har bir bosqichida sarflanayotgan quyosh energiyasi ketma-ket taqsimlanib boradi. Metabolitik jarayon mahsuli bo'lgan organik qoldiq produtsentlar tomonidan qaytadan o'zlashtiriladigan holatga o'tadi.

Jamoalarda energiya va moddalar oqimi jarayonida redutsentlar asosiy ahamiyatga ega. Fotosintez natijasida suv va karbonat angidrid bog'langan holga o'tadi va undagi kimyoviy elementlar turli organik birikmalarning muhim tarkibiy qismiga aylanadi. Shunday qilib, ular boshqa organizmlar o'zlashtira olishi mumkin bo'lgan holga o'tadi. Konsumentlarning metabolitik mahsuloti sifatida karbonat angidrid yana atmosferaga qaytadi. Konsumentlardan ajralayotgan energiya ularning tana haroratini bir me'yorda ushlab turish imkoniyatini beradi. Ortiqcha energiya issiqlik va yorug'lik holda atmosferaga singib ketadi. Atmosfera tarkibidagi kabonat angidrid va boshqa biogen elementlar (azot, fosfor, oltingugurt va boshqalar) o'simlik tomonidan qaytadan o'zlashtiriladi. Shundan so'ng energiya oqimining yangi tsikli boshlanadi.

Energiya quyosh radiatsiyasi sifatida erga etib keladi va o'simliklar tomonidan bog'langan energiyaga aylantiriladi. Ozuqa zanjiri bo'ylab bog'langan kimyoviy energiyaning sarfi ro’y beradi, sarflanmagan energiya samarasiz issiqlik sifatida yo'qotiladi. Organik qoldiq esa, ta'kidlanganidek, 
redutsentlar faoliyati natijasida parchalanib boshqa ko'rinishga o'tadi. Quyoshdan kelayotgan energiya hisobiga erdagi hayot mavjud bo'ladi. Kimyoviy elementlar turli moddalar tarkibiga kirib, bog'langan holda o'simlik va hayvonlar tomonidan o'zlashtiriladi. Umuman, biogeotsenoz va ekotizmlar hamda ularning muhim tarkibiy asosini tashkil etuvchi jamoalarni Klimentes talqiniga ko'ra «yuqori organizm» darajasidagi birlik sifatida qarash mumkin.

\section{Ekologik tokchalarning ajralishi}

Ekotizimda har bir tur o'z o'rniga ega va ma'lum vaqt oralig'ida yashaydi, shu xususiyat bilan u boshqa turlardan farqlanib turadi. Turning atrof-muhitga tobeligi uning geografik areal bo'ylab tarqalishi va turli yashash joylarini egallashida o'z ifodasini topadi. Yashash joyida turning tabiiy jamoalar bilan funktsional bog'lab turuvchi fazo vaqtidagi o'rni ekologik tokcha deyiladi. Har qanday jamoada turlararo raqobat ma'lum darajada barqarorlashgan bo'lishi lozim.

Jamoalar strukturasiga raqobatning ta'sir etishi. Turlararo raqobat jamoalarda muhim o'rin tutadi. Ma'lumki, ekologik talab darajasi yaqin turlar bir ozuqa resursi uchun raqobatda bo'lganda, oxir oqibatda eng yuqori imkoniyatga ega bo'lgan turgina saqlanib, qolganlarini siqib chiqaradi va qurbon qiladi (Gauze qoidasi).

Jamoalarda ekologik talab darajasi yaqin turlar har xil ekologik tokchani egallaydi. $\mathrm{Bu}$ jihatdan raqobat asosida ekologik tokchalarning ajralishi jamoalarning tashkillanishi va shakllanishiga sharoit yaratadi.

Jamoadagi nisbatan bir xil ekologik sharoit turlararo raqobatni yuzaga keltiradi va u jamoa strukturasiga sezilarli ta'sir etadi. Lekin jamoalardagi yirtqichlik, sifatsiz ozuqa manbasi, noqulay iqlim sharoitlari populyatsiya zichligini ancha past darajada ushlab turadiki, bu holda turlararo raqobatga ortiqcha o'rin qoldirmaydi. Aniqlanishicha, quruqlikdagilarga nisbatan dengiz hayvonlarida turlararo raqobat etakchilik qiladi, mayda hayvonlarga nisbatan yiriklarida turlararo raqobat kuchli ketadi.

O'simlikxo'r hasharotlar - fitofaglarning bir necha turi bir o'simlikda oziqlanganda, ularning har biri turli ekologik tokchani egallaydi (novda uchi, barglar, tanasi, tomir va hokazo). Fitofaglar populyatsiyasining zichligi 
yirtqich va parazitlar faoliyatiga, ozuqa sifatiga, shuningdek, o'simlikning himoyalanish darajasiga muvofiq holda yuqorilashib ketmaydi. Bularning barchasi fitofaglar o'rtasidagi raqobatni mo"tadillashishiga sabab bo'ladi. Lekin shunga qaramay, Atkinson va Shorrokslarning (1981) immitatsion matematik modeliga muvofiq turlararo raqobat mavjud. Jumladan, efemer ozuqa uchun kurashayotgan ikki tur o'simlikda yoppasiga emas (masalan, bir barg plastinkasida), balki hol-hol bo'lib joylashadi. Bunday guruhlanish chegaralangan ozuqa manbasidan ikkala tur ham bir vaqtda foydalanish imkoniyatini yaratadi. Masalan, olma bargidagi yashil olma shirasi va olmaotquloq shirasi koloniyalari ayni shunday guruhlar hosil qilib joylashadi. Olma-otquloq shirasi olmada juda qisqa vaqt yashaydi va otquloqqa uchib o'tadi. Olmada qolgan tur ko'plab ko'payish imkoniyatiga ega bo'ladi. Bunday holda turlararo raqobat yo'qday ko'rinadi, lekin dastlab ular o'rtasida raqobat bo'lganligi tabiiy, keyingi tanlanish bu raqobatni mo"tadillashtirishga olib kelgan. Bu holatni Konnel "Dastlabki raqobatning sharpasi"deb nomlangan. Tabiiy tanlanishda raqobatga bardosh bera olmagan turlar "O'yindan chiqqan" va hozirda turlararo raqobat mo"tadillashgan sharoitda yashashga moslashgan turlar saqlanib qolgan. Lekin jamoalarda $u$ yoki bu turning yoppasiga ko'plab ko'payishi bilan turlararo raqobat kuchayib boradi. Shuni ta'kidlash lozimki:

a) bir jamoadagi raqobatdagi ikki tur eng minimal darajada bo'lsada, ekologik tokchalarning ajralishini namoyon etadi;

b) ekologik tokchalarning ajralishi morfologik differentsiyalanishda o'z ifodasini topadi;

v) har qanday jamoada bir xil ekologik tokchani egallovchi ikki tur yashay olmaydi, ularning biri albatta bo'sh yashash joyini egallashga harakat qiladi.

Ekologik tokchalarning ajralishi, turli yo'nalishlarda o'z ifodasini topadi. U mavjud yashash joyida bir qancha mayda yashash joylarini shakllanishiga sabab bo'ladi. Natijada, har bir mikro yashash joyi o'zining iqlimiy va boshqa ekologik sharoitlari bilan ajralib turadi.

Ekologik tokchalar hayvonlarning yashash joylari (barg, poya, novda, tomir va boshqalar), o'simlikdagi yashash balandligi bo'yicha, tana o'lchamlari, populyatsiya zichligi va yashash davri bilan farqlanadi. Bu holatni 
gulpar o'simligida hayot kechiradigan shiralarning 4 turi misolida ham ko'rish mumkin.

Unda ifodalanishicha, eng katta o'lchamli tur o'simlikning tomir bo'g'izida, unga nisbatan kichikroq o'lchamdagi tur barg qo'ltig'ida yashaydi. Keyingi ikki tur esa gul bandlari va savatchalarida hayot kechiradi. Yuqori yarusda eng mayda shiralar hayot kechiradi. Tomir bo'g'izidagi shiralar 3-4 ta bo'lib yashasa, eng yuqori yarusdagi shiralar xartumchasining uzunligi bo'yicha tomir bo'g'iz shirasi alohida ajralib turadi, yuqori yarusdagi shiralarning xartumchasining uzunligi bir muncha kalta bo'ladi. Gulpar shiralaribir gildiyaga mansub bo'lib, bir o'simlikdan ozuqa manbasi sifatida foydalanishga ixtisoslashgan. Agar turlararo raqobat mavjud bo'lsa, yoki dastlab bo'lgan bo'lsa, u holda raqobat aynan gildiyalar ichida sodir bo'ladi. Tabiatda bir gildiyaga mansub turlar bir taksonomik guruhga yoki bir necha taksonomik guruhga mansub bo'lishi mumkin.

Jamoalarda ekologik tokchalarning ajralishi va tarlararo raqobatning mo"tadillashishi hayvonlarni ozuqa resurslaridan unumli foydalanishga imkoniyat yaratadi.

3-jadval

\section{Gulpar o'simligida ekologik tokchalarning ajralish ko'rsatkichlari}

\begin{tabular}{|c|c|c|c|c|c|}
\hline \multirow[b]{2}{*}{ Shiraning nomi } & \multirow[b]{2}{*}{ oziqlanish joyi } & \multirow{2}{*}{$\begin{array}{c}\text { qavat } \\
\text { balandligi } \\
\text { (sm da ) }\end{array}$} & \multicolumn{3}{|c|}{$\begin{array}{l}\text { Shiralarning o'lchamlari } \\
\qquad \mathrm{x}, \mathrm{n}=20\end{array}$} \\
\hline & & & tanasi & $\begin{array}{l}\text { xartum- } \\
\text { chasi }\end{array}$ & $\begin{array}{l}\text { xartumcha } \\
\text { sining } \\
\text { 4-bo'g'imi }\end{array}$ \\
\hline $\begin{array}{l}\text { Anuraphis } \\
\text { subterranea (Walk.) }\end{array}$ & Tomir bo'g'izi & $0-5$ & $3,22 \times 2,21$ & 1,53 & 0,43 \\
\hline $\begin{array}{l}\text { Dysaphis crataegi } \\
\text { (Kalt.) }\end{array}$ & Barg qo'ltig'i & $10-15$ & $2,31 \times 1,29$ & 0,80 & 0,13 \\
\hline $\begin{array}{l}\text { Cavariella theobaldi } \\
\text { (Gill. et Bragg.) }\end{array}$ & Gul bandlari & $80-90$ & $1,92 \times 0,98$ & 0,64 & 0,12 \\
\hline $\begin{array}{l}\text { Hyadaphis passerinii } \\
\text { (del Guerc) }\end{array}$ & Gul savatchasi & $100-110$ & $1,72 \times 0,88$ & 0,62 & 0,10 \\
\hline
\end{tabular}

\section{Ekotizimlarning o'zgarishi}

Ekotizimlarning barqarorligi nisbiy bo'lib, vaqt mobaynida o'zgarib 
boradi. Bunda jamoalarning tur tarkibi, undagi munosabatlar, yashash sharoitlari o'zgaradi. Natijada, biotsenoz o'zining o'tmishdoshidan son va sifat jihatdan farqlanishi ro'y beradi. Ekotizimlarning vaqt o'tishi bilan birining ikkinchisi bilan almashishi hodisasi ekologik suktsessiya deb nomlanadi.

Jamoalarda davriy-tsiklik o'zgarishlar ro'y berishi mumkin. Hayvonlar hayotidagi kunlik va mavsumiy o'zgarishlar jamoalarda ham o'z ifodasini topadi.

Ekologik o'zgarishlar uch guruhga bo'linadi: a) degradatsion, b) allogen, v) avtogen o'zgarishlar.

Degradatsion o'zgarishlar turli vaqt oralig'ida sodir bo'lishi mumkin. Barcha tirik organizmlarning qoldiqlari va jasadlari erga tushib mikroorganizmlar ta'sirida mineral qoldiqqa aylanadi. $\mathrm{Bu}$ jarayoning borishida ketma-ket bir necha geterotroflar ishtirok etadi, shuning uchun bu o'zgarishni geterotrof suktsessiya deb ham nomlanadi. Geterotrof o'zgarish, resursni to'liq o'zlashtirilishi va minerallanishi bilan xotima topadi. Masalan, tog'-o'rmonlari yoki daraxtzorlar tagiga tushgan barglar, shox-shabbadan iborat to'shamaning qalinligiga bog'liq. To'shama ostida chirish boshlangan bo'lsa, yuzasida bu jarayon dastlabki bosqichda bo'ladi. To'shamada bir necha turga mansub zamburug'lar ketma-ket yashaydi, zamburug'lar faoliyati tugallangan qavatda tuproq kanalari uchun qulay sharoit yuzaga keladi. Ular o'zlashtirib parchalagan qoldiq boshqa hayvonlar yashashi uchun qulaylik tug'diradi. Bir yil o'tgandan so'ng to'shamaning chuqur qatlamlarida dumoyoqlilar, kanalar, chuvalchanglar va boshqa umurtqasizlar ko'plab ko'payadi. Shu ketma-ketlikda davom etgan suktsessiya jarayoni yillar davomida ro'y beradi. Suktsessiya jarayonida hosil bo'lgan organik va mineral moddalar tuproq bilan aralashib, uning unumdorligini oshiradi. Organizmlarning qoldiqlari (chiqindilari, jasadlari, barg va boshqalar) erga tushib jadal degradatsiyalanmaydi, aksincha yangi yashash joyini vujudga keltiradi. Qoldiqlarni turli o'troq organizmlar egallab olib yashay boshlaydi. Ma'lum muddat o'tgandan so'ngina shu joyda boshqa yo'nalishdagi o'zgarishlar sodir bo'lishi mumkin.

Avtogen o'zgarishlar biologik jarayonlarning mahsuli bo'lsa, allogen suktsessiyalar tashqi geofizik va kimyoviy sharoitlarni o'zgarishi natijasida ro'y beradi. 
Allogen o'zgarishlar natijasida, jumladan, suv havzalarning quyi oqimlarni loyqa bilan to'lishi, botqoqliklarni qurib borishi bilan ular o'rnida o'simliklar qoplami shakllanadi. Daraxt va butalar uchun qulay sharoit yaratiladi. Allogen o'zgarishlar abiotik omillar (loyqa, ta'siri va boshqalar)ga bog'liq amalga oshadi.

Jamoalarning asta-sekin o'zgarishlari natijasida bir jamoa ikkinchisi bilan almashinadi. Bunday o'zgarishlarning sababi jamoaga uzoq vaqt davomida tashqaridan ma'lum yo'nalishidagi omilning ta'siri natijasidir. Ekotizimlardagi bunday almashinish avtogen o'zgarish deb ataladi. Avtogen suktsessiya birlamchi va ikkilamchi bo'lishi mumkin. Suktsessiya o'simlik va hayvonlardan butunlay holi bo'lgan joylardan boshlansa, birlamchi o'zgarishlar kelib chiqdi. Muhit sharoitlarining o'zgarishi bilan bir jamoaning ikkinchisi bilan almashinishi ikkilamchi suktsessiya hisoblanadi.

Jamoaning birlamchi shakllanishi qurib qolgan ko'l va dengiz o'rnida, qumli yotqiziqlarida, yalang'och qoyalar va qoyatoshlarda, tosh-shag'alli joylarda kuzatiladi. Bu joylarda dastlabki, tasodifiy kelib qolgan urug'lardan ungan o'simliklar yashaydi, keyinchalik hayoti o'simliklar bilan bog'liq hayvonlar jamlanadi. Umuman, birlamchi suktsessiyada dastlabki bosqich, guruhlanish va jamoaning barqaror shakllanish davrlari kuzatiladi. Jamoalardagi ikkilamchi suktsessiyalar tashqi muhitning, yashash sharoitining asta-sekin yoki keskin o'zgarishlari natijasida sodir bo'ladi.

Iqlim, tuproq, tirik organizmlar va antropogen omil jamoalarga ta'sir etishi mumkin. Masalan, yangi erlarning o'zlashtirilishi bilan agrotsenozlarning shakllanishi ikkilamchi suktsessiya natijasida hisoblanadi. Tabiatdagi turli falokatlar va halokatlar (er silkinishlari, vulqonlar harakati, to'fonlar, yog'inlar va boshqalar) natijasida jamoalarning almashinishi, turlarning nobud bo'lishi va keyinchalik asta-sekin tiklanishi kuzatiladi.

Jamoadagi o'zaro tashqi muhit bilan bo'lgan munosabatlarning uyg'unligi davri klimaks deb nomlanadi. Jamoaning klimaks davri nisbiy bo'lib, jamoadagi tashqi ichki omillar ta'sirida buzilishi mumkin. 


\section{5-BO'LIM. BIOSFERADA HAYVONLARNING \\ TARQALISH CHEGARALARI}

Er sharining tirik organizmlar tarqalgan qobig'i (atmosfera, gidrosfera, litosfera) biosfera deb nomlanadi. Biosfera hayot qobig'i bo'lib, bir-biri bilan murakkab munosabatlar orqali bog'langan bir qancha ekotizimlar majmuidan iborat. Biosfera tarkibiga hozirda quruqlikda, havoda va suvda tarqalgan tirik organizmlargina emas, balki qadimda yashab, hozirda qirilib ketgan organizmlar ishtirokida shakllangan litosferaning qismi ham kiradi.

Hayvonlar suv va quruqlikda yashaydi, hayoti to'lig'icha havo muhiti bilan bog'langan hayvonlar biosferada uchramaydi. Havoda faol (qushlar, sut emizuvchilar) yoki passiv (mayda hasharotlar, sporalar, tsistalar) uchib moslashgan hayvonlarning oziqlanishi, ko'payishi quruqlik yoki suv muhitida boradi.

Biosferaning yuqori chegarasi $12-17 \mathrm{~km}$ balandlikda o'tadi. Uning yuqori qatlamlarida bakteriyalar, sporalar, sodda hayvonlar va boshqalar uchraydi. Biosferaning yuqori qismi ozon qatlami bilan o'ralgan. Ozon qatlami organizmlar uchun zararli bo'lgan ultrabinafsha va koinot nurlarini tutib qoladi. Ozon qatlami er yuzasidan 50-70 km yuqorida joylashgan. Quruqlik hayvonlarining ko'pchiligi, aksariyat hollarda havoda 50-100 m balandlikdan yuqoriga ko'tarila olmaydi. Er yuzasidan $100 \mathrm{~m}$ balandlikda hayvonlar kam uchraydi. Lekin, ayrim qushlar safar uchishlari davomida 1000-3000m balandlikda parvoz qiladilar, hasharotlar 4000-5000 m yuqorida, havo oqimi bilan harakatda bo'ladi. Markaziy Osiyo tog'larida hayvonlar dengiz sathidan 4000-5000m balandlikda yashaydi. Masalan, yovvoyi qo'ylar - arxarlar tog' etagidagi erlardan tortib, to $5500 \mathrm{~m}$ balandlikkacha tarqalgan. Zag'chalar Everestning $8200 \mathrm{~m}$ balandligida ham uchrashligi qayd etilgan.

Biosferaning quyi chegarasi 2-3 km chuqurlikdan o'tadi. Neft yotqiziqlarining suvlaridan topilgan mikroorganizmlar shundan dalolat beradi. Hayvonlarning eng chuqur inlari 6-7 $\mathrm{m}$ da joylashadi. Masalan, yumronqoziq va sug'urlarning inlari 6-7 metrda, termitlar uyalarda 5-6 m chuqurlikda bo'ladi. Ko'pchilik hayvonlar litosferaning bir metrga qadar bo'lgan qatlami bo'ylab taqsimlangan. Gidrosferada hayot tarqalgan eng quyi nuqta Tinch okeanida, $1100 \mathrm{~m}$ chuqurlikda qayd etilgan. Tinch okeanining bu 
mintaqasi turlarga boy bo'lib, ayniqsa pogonoforalar ko'plab uchraydi. Suv hayvonlarining ko'pchiligi suv yuzasining 300-500 m qalinligi bo'ylab jamlangan.

Biosfera doirasida ayrim o'ta sho'rlangan suv havzalari (O'lik dengiz, Armaniston Tuz ko'l), vodorod sulfid, karbonat angidrid kabilar bilan zaharlangan hududlardagina tirik organizmlar deyarli uchramaydi. Lekin, mazkur sharoitda ham ayrim bakteriyalar, sporalar va tsistalar qayd etilganligi ma'lum.

Biosfera Er sharidagi global ekotizim bo'lib, u bir qancha kenja tizimlar biogeotsenozlar birligidan tarkib torgan.

Biosfera to'g'risidagi ta'limotda akademik V.I.Vernadskiy hozirgi vaqtda insoniyat Erning yangi qobig'ini - noosfera («oqilona qobiq»)ni yaratishi kerak deb taxmin qiladi. Insoniyat biosferadagi qiyosan kichik bir massadir, lekin uning faoliyati g'oyat ulug'vor. Inson endilikda biosfera doirasidan tashqariga chiqib oldi, uning kosmik kemalari $0 y$, Venera va boshqa planetalarga etib bordi.

\section{Biosfera va insoniyatning amaliy faoliyati}

Inson eng kuchli tabiiy omil sifatida biosferada muhim ahamiyatga ega. Ibtidoiy davrda faoliyatning tabiatga ta'siri sezilarsiz bo'lgan bo'lsa, kishilik jamiyatining rivojlanib borishi, bir ijtimoiy-iqtisodiy formatsiyadan ikkinchisiga o'tish, aholi sonini ortib borishi, ishlab chiqarish kuchlarining ravnaqi, fan-texnika va qishloq xo'jaligining jadal taraqqiyoti antropogen omilni biosferaga bo'lgan ta'sirini ortishiga sabab bo'ldi.

Antropogen omil tabiatga bevosita va bilvosita ta'sir o'tkazadi. Jumladan, ayrim turga mansub organizmlarning yo'q bo'lib ketishi, ba'zilarning esa keskin ko'payishi, turli mintaqalar bo'ylab yangi ekologik majmualarning shakllanishi antropogen omilning tabiatga bo'lgan to'g'ridanto'g'ri ta'sirini ifodalaydi. Inson faoliyati natijasida organizmlarning yashash sharoiti va muhitining o'zgarishi (iqlim, gidrologik holat, tuproq strukturasining o'zgarishi) antropogen omilning nisbiy ta'siri hisoblanadi.

Inson faoliyati tabiatda katta ijobiy ahamiyatga ega, lekin u biosfera tizimidagi salbiy o'zgarishlarga ham sabab bo'ladi. Masalan, O'zbekistonda 
dehqonchilikning jadal rivojlanishi tabiiy yashash sharoitining to'liq tanazzulga yuz tutishiga, kimyoviy moddalarning yuqori darajada, tartibsiz qo'llanilgani, paxta yakka hokimligi, erlarning o'ta sho'rlanganligi sababli barcha haydalgan er maydonlarining bioxilma-xilligi nihoyatda past darajaga tushib qolishiga sabab bo'ldi. Garchi sug'oriladigan erlar barcha hududlarning $10 \%$ ni tashkil etsa-da, O'zbekiston va unga chegaradosh respublikalarning ekologiyasiga katta ta'sir ko'rsatib, ba'zi bir ekotizimlarning to'liq buzilishiga va ayrim biogeotsenozlarning keskin qisqarishida namoyon bo'ldi. Barcha ekotizmlar amalda katta o'zgarishlarga uchradi.

O'zbekiston tabiiy o'simliklar qoplami va o'rmonlar dasht va cho'l mintaqalarining 85\%, tog'larning 13\% ni egallaydi. Daryo bo'ylari va vodiylarida ularning bori yo'g'i $2 \%$ qolgan. To'qayzorlar yaqin vaqtga qadar katta hududlarni egallagan, lekin daraxtlarning kesilishi daryo o'zanlarini to'g'rilash oqibatida bu maydonlar 10 martadan ko'pga qisqarib ketdi.

O'zbekistonda tog' o'rmonlarning tartibsiz kesilishi, Amudaryo va Sirdaryo bo'ylaridagi tabiiy to'qayzorlarni o'rilishi ayni hududlardagi ekologik sharoitni keskin o'zgarishi va gidrosfera muvozanatini saqlovchi omildir. Lekin, hech bir o'ylamasdan o'rmon va to'qayzorlarni yo'qotilishi tuproqning yuvilishiga, ikkilamchi sho'rlanishga, jarlarning hosil bo'lishiga, qum bosishi va hayvonlarning qirilishiga sabab bo'ladi. Vaqt o'tishi bilan mazkur mintaqalarda yangi o'simliklar qoplami vujudga kelishi kuzatildi. Juda katta tanazzullar, sho'rlanish, botqoqlanish va kimyoviy zaharlar bilan ifloslanish, qishloq xo'jalik amaliyotida ekologik asoslanmagan agrotexnik va himoya usullarini qo'llash natijasida kelib chiqqan.

Tabiiy ekotizmlarning o'zgarishi ko'pchilik hayvonlarni, birinchi navbatda, yirik sut emizuvchilarni yo'q qilinishiga sabab bo'ldi. Yirtqichlarning yo'qolib ketgan turlariga turon yo'lbarsi, yo'qolish arafasida bo'lgan turlarga yo'l-yo'l sirtlon, qoraquloq, Osiyo qoploni mansub. Respublika hududida uchraydigan 184 tur hayvonlar noyob va yo'qolib ketish arafasida ekanligi «Qizil kitob» nashrida alohida ta'kidlagan. Sut emuzuvchilarning 23 turi ( 1 ta kenja turi), qushlarning - 48 turi ( 3 ta kenja turi), sudralib yuruvchilarning - 16, baliqlarning 17 turi (1 ta kenja turi) va umurtqasizlardan 78 tur ushbu kitobdan o'rin olgan.

Inson yangi zot va navlarni keltirib chiqarish, o'simlik va hayvonlarni 
iqlimlashtirish asosida tabiatni bir muncha boyitadi.

1960 yillarda O'zbekistonda 40 turga yaqin baliqlar uchraganligi ma'lum. Keyingi 40 yil davomida turli maqsadlarda olib kelinib iqlimlashtirilgan baliqlar hisobiga ularning xilma-xilligi 80 turga qadar ortdi. Shu bilan birga, ayrim mahalliy baliqlarning yo'qolib ketish xavfi tug'ildi, ba'zilari xatto yo'qolib ketdi. Hayvonlarni iqlimlashtirish tajribalari hamma vaqt ham ijobiy natija beravermaydi. Keltirilgan ayrim turlar mahalliy sharoitga moslasha olmay, qirilib yoki ayni hududdagi ekologik sharoitning mos kelishi tufayli ular ko'plab ko'payib jiddiy zarar keltirishi ham mumkin.

Insonning biosferaga ko'rsatadigan ta'sirlaridan biri qishloq xo'jalik ekinlari, hasharotlar va begona o'tlarga qarshi kimyoviy kurash usulidan keng foydalanishdir. Kimyoviy zaharlar va zararli moddalar kasalliklarni bartaraf etish, begona o'tlarni qirish, zararli hasharotlar miqdor zichligining keskin kamayishida muhim vosita bo'lib xizmat qiladi. Lekin tartibsiz va yoppasiga qo'llaniladigan kimyoviy moddalar biogeotsenozlarda uzoq vaqt, barqaror saqlanib qoladi hamda to'planadi. Ozuqa zanjiri bo'ylab harakatda bo'lgan zaharli va zararli moddalar turli hayvonlar va odamga salbiy ta'sir etadi.

Kelajakda o'simliklarni himoya qilishda biologik nazorat va kurash usullarini keng joriy etish ijobiy samara berishi shubhasiz. Zararkunandalarga qarshi bilogik usullardan foydalanishga barcha mintaqalarga katta e'tibor berilmoqda.

Inson tabiatning bir qismi sifatida biosferaga bo'lgan munosabatini butunlay o'zgartirishi, uning boyliklaridan oqilona foydalanish, atrof-muhit musaffoligini saqlashda muhim o'rin tutadi. Inson faoliyati biosferani yangi sharoitda uzoq va barqaror funktsiyalanishiga ko'maklashishi, bu jarayonlar qonuniyatlarini tadqiq etishga qaratilishi lozim. Ekologik toza qishloq xo'jalik mahsulotlarini etishtirish, barqaror agroekotizimlarni shakllantirish, o'simliklarni himoya qilishda foydali turlarning tabiiy populyatsiyalaridan keng foydalanishga e'tibor berish lozim.

Baliqchilik va ov xo'jaliklari faoliyatlarini ekologik ilmiy asosga qo'yish, tabiat boyliklaridan unumli foydalanish bilan ularni tiklash borasida keng amaliy faoliyat ko'rsatish zarur. Bugungi kunda buzilgan ekotizmlarning tiklanishi va qayta shakllanishiga sharoit yaratish, buning uchun suktsessiya jarayonlari qonuniyatlarini bilish va amalda qo'llash taqozo etiladi. 
Inson amaliy faoliyatidagi biosferaga bo'lgan salbiy ta'sirini bartaraf etish va oldinda turgan ulkan ekologik vazifalarni bajarish malakali kadrlarni tayorlash va ularning salohiyati bilan belgilanadi.

\section{Zararkunanda hasharotlarga qarshi kurashning biologik usuli}

Ma'lumki, hasharotlar nihoyat darajada serpusht va tez ko'payuvchi organizmlardir. Lekin, obi-havo sharoitlari, foydali va zararli organizmlar o'rtasida shakllangan evolyutsion munosabatlar hasharotlarni keskin ko'payib ketishini chegaralab turadi. Garchand umumiy ko'payishga nisbatan tabiatda «Oz» miqdorda zararkunanda hasharot qolsa-da, ular qishloq ho'jaligi ekinlariga katta zarar keltiradi. Masalan, g'o'za zararkunandalari hamda kasalliklari ko'payganda, ularga qarshi keskin choralar ko'rilmasa, etishtirilgan paxta hosilining $25-40 \%$ i nobud bo'ladi. Kuzatishlar shuni ko'rsatadiki, bitta ko'sak qurti 20 ta hosil elementini 2-4 ta ko'sakni zararlaydi. G'o'za shiralari esa paxta hosilini o'rta hisobda 1,5\% gacha kamaytirib yuborishi mumkin. Shaftoli daraxti tanasida yashaydigan shira esa yil davomida bitta daraxtdan 50-550 kg sof qand moddasini o'zlashtiradi. Zararkunanda hasharotlarga qarshi kurash uchun sarflanadigan mablag' bir necha yuz millionni tashkil qiladi. Ularga qarshi kurash choralarini takomillashtirish, iqtisodiy arzon va atrof-muhit uchun xavfsiz kurash usullarini ishlab chiqish mutaxassislar va olimlar oldida turgan muhim vazifadir.

Keyingi yillarda zararkunandalarga qarshi ishlatiladigan kimyoviy vositalarning (insektitsidlarning) turlari ko'paytirilmoqda. Bu dorilar yuqori samarali bo'lishi bilan birga, dalalardagi foydali hasharotlarni qirilishiga sabab bo'ladi. Shu bilan bir qatorda, issiq qonli hayvonlar, inson organizmiga ta'sir etadi hamda atrof-muhitni ifloslaydi.

Hozirgi davrda zararkunandalarga qarshi biologik kurash usuliga katta e'tibor berilmoqda. Biologik kurash usuli zararli hasharotlarni tabiiy dushmanlari - foydali hasharotlar, hasharotxo'r qushlar, mikroorganizmlar va boshqa hayvonlar yordamida qirishidir. $\mathrm{Bu}$ usul kimyoviy dorilar qo'llanilishini keskin kamaytiradi va muxofazalanishida muhim rol o'ynaydi.

O'simliklarni zararkunanda hasharotlardan biologik yo'l bilan himoya 
qilish yangilik emas, undan u yoki bu ko'rinishda insonlar ko'pdan beri foydalanib kelganlar. $\mathrm{Bu}$ usulga oid fikrlarni rivojlanishi o'tgan asrning o'rtalariga to'g'ri kelsa, «beminnat yordamchilardan» rejali foydalanish asrimizning ikkinchi yarmida amalga oshdi. Hozirda bu usul qishloq xo'jaligida juda keng qo'llanilmoqda.

Hozirgi paytda biologik kurash maydonidagi asosiy himoyachi bu trixogrammadir. U mayda qanotli hasharot bo'lib, etuklik davrida gul nektari bilan ovqatlanadi, asosan, tunlamlarning tuxumi ichiga o'z tuxumini qo'yib rivojlanadi. Parazit tuxumidan chiqqan lichinka o'lja tuxumi hisobiga voyaga etadi, g'umbakka va etuk formaga aylanadi. Bu hasharot tabiatda erkin ko'paya oladi, lekin ularni tabiatdagi soni zararkunandalarni to'lig'icha daf qilishiga qurbi etmaydi. Shu sababli trixogrammalarni maxsus biolaboratoriyalarda sun'iy ko'paytirilib, dalalarga olib chiqib tarqatiladi. Bugungi kunda O’zbekistonda bunday laboratoriyalarning soni 800 ga yaqinni tashkil etadi.

Ko'sak qurtiga qarshi kurashda keng qo'llanilayotgan ikkinchi parazit bu yaydoqchi gabrobrakondir. Urg'ochisi o'rta yoshdagi ko'sak qurtini tanasiga tuxum qo'yadi, chiqqan lichinkalar o'z o'ljasi hisobiga voyaga etadi. Yaydoqchilar o'simlik nektari bilan ovqatlanadi, shuning uchun ularning tabiatdagi sonini orttirish yo'llaridan biri nektarga boy o'simliklarni ekishdir. Shiningdek parazit laboratoriya sharoitida ham ko'paytirilib dalalarga tarqatiladi.

O'rgimchakkanaga qarshi kurashda yirtqich kana - fitoseyulyus qo'l kelmoqda. Ma'lumki, issiqxona sharoitida o'rgimchakkana 10-25 ta avlod beradi. Bu esa, ayniqsa bodring hosiliga katta xavf tug'diradi. Kimyoviy dorilarning qo'llanilishi iqtisodiy nomuvofiq bo'lishi bilan birga, bodring mevasida yig'ilishi mumkin. Bunday sharoitda zarakunandalarga qarshi kimyoviy zaharlar o'rnini bosadigan, tabiiy yordamchi - yirtqich kana tarqatiladi. U o'rgimchakkananing etuk formalarini emas, balki tuxumlarini ham faol qiradi. Bir dona kana 24 tagacha zararkunandani yoki 30 tagacha tuxumini eyishi mumkin.

Kechalari chiroq yorug'iga keladigan nozik yashil hasharotlar oltinko'zlardir. Urg'ochilari o'z tuxumlarini o'simlik barglarining yuqoritomonida 15-30 tagacha, ba'zan 2-3 tadan maxsus poyaga qo'yadi. 
Lichinkalari nihoyatda harakatchan bo'ladi. Ular juda ko'plab shiralar, qalqondorlar, o'simlik kanalari, tunlam va kichik yoshdagi qurtlar bilan ovqatlanadi. Masalan, oltinko'z lichinkasi 1 soatda 30 ta o'rgimchakkanani va hayoti davomida 4000 shirani yo'qota oladi. Issiqxona xo'jaliklarida bu hasharotdan ancha keng foydalanilmoqda, keyinchalik ularni sun'iy usulda ko'paytirish keng yo'lga qo'yilgan, u o'simlik himoyachilarining 1yordamchisiga aylanishi mumkin.

Keyingi paytlarda issiqxona xo'jaliklarida yirtqich gallitsa -qo'llash yo'lga qo'yildi. Bu hasharotlar 60 turdan ortiq shiralar bilan oziqlanadi. Ularning lichinkasi 1 kunda 65 taga qadar shirani yo'q qiladi.

Yuqorida sanab o'tilgan foydali xasharotlar o'simlik himoyasida qo'llanilayotgan biologik agentlarning ayrimlari xolos. Tabiat bizga qator «beminnat yordamchilar»ni berganki, hozirda ulardan foydalanish yo'llari ustida olimlarimiz ishlamoqdalar. Jumladan, bug'doyning xavfli zararkunandasi hasvaga qarshi yirtqich qo'ng'izlar telenominlar va fazi pashshalar (o'z tuxumini hasvaning ko'ziga yopishtirib qo'yadi)ni qo'llash katta naf keltirgan bo'lar edi. Shuningdek, eng foydali hasharot xonqizi qo'ng'izlari biologik agentlar qatoriga qo'shilganicha yo'q. O'zbekiston respublikasining o'zida 30 turdan ortiq xonqizi qo'ng'izlari uchraydi, shulardan faqatgina 2 tasi zararkunanda xolos. Qolgan barchasi, paxta dalalarida, bog'larda va boshqa maydonlarda shiralar, tunlamlar sonini kamaytirib turishda katta rol o'ynaydi. Ayniqsa, sitsimnus qo'ng'izlari laboratoriya sharoitida oson ko'payadi. Urg'ochi qo'ng'iz o'rtacha 245 ga qadar tuxum qo'yadi. Lichinkasi 1 kunda 33-37 ta, etuk formasi 15-25 ta shira bilan oziqlanib, lichinka voyaga etguncha qadar 300 mingdan ortiq zararkunandani yo'q qiladi.

Foydali bo'g'imoyoqlilardan yana biri - sirfid pashshalaridir. Ko'payishi arilarni eslatuvchi bu hasharotlar etuklik davrida gullarni shirin nektari bilan ovqatlanadi, lichinkalari esa asosan shiralarga qiron keltiradi. Lichinkalari kam harakat, ko'rinishi zuluklarni eslatadi (kattaligi $1 \mathrm{sm}$ ga boradi). Yashil zarg'aldoq yoki qizil rangda bo'ladi. Ular 1 kunda 200 tadan ortiq shirani yo'q qiladi.

Biologik kurash usulining keyingi yo'nalishi bu foydali hasharotlarni iqlimlashtirishdir. Qizil qon shirasi paraziti afelinus bunga misol bo'la oladi. 
Bu hasharot XX asrning 20-yillarida Shimoliy Amerikadan dunyoning 40 mamlakatiga tarqatilgan. Hozirga paytda bu parazit qizil qon shirasi tarqalgan barcha tabiiy hududlarda uchraydi. Bahorda olma daraxtlarini yosh novdalari va tanasining yorilgan joylarida oppoq g'uborni ko'rishimiz mumkin. Bu qizil qon shirasidir. Agar bu zarakunanda ko'payib ketsa, meva daraxtiga jiddiy zarar etkazadi. Lekin uning koloniyalari orasida mayda hasharot afelinuslar o'z tuxumini zararkunanda tanasiga qo'yib, ular sonini keskin kamayishiga sabab bo'ladi. Shuning uchun ham yozning 2-yarmida oq g'uborlar qolmaydi. Ular o'rnida nobud bo'lgan shiralar qoladi. Afelinus tabiatda qizil qon shirasi sonini olmagan iqtisodiy zarar beradigan darajaga ko'tarilishiga yo'l qo'ymaydi.

1939 yilda Toshkentda mevali daraxtlarva tutning havfli, karantin zararkunandasi - komstok qurti topilgan edi. Yildan-yilga u keng maydonlarni egallab zarar keltira boshladi. Ipakchilik xavf ostida qoldi. 1945 yilda bu «chaqirilmagan mehmon»ga qarshi uning parazit - psevdofikus olib kelindi. Bu kichik hasharot tezda o'z faoliyatini boshlab yubordi va 60-yillarga kelib (komstokka) qarshi kimyoviy vositalarni qo'llashga ehtiyoj ham qolmadi.

Yirtqichkana - metaseyluyus o'rgimchakkana bilan oziqlanishga ixtisoslashgan akarifagdir. Urg'ochisi hayoti davomida 75-80, erkagi 30 dan ortiq o'rgimchakkana bilan oziqlanadi. Metaseyluyus iqlimlashtirilgach, mevali bog'larda o'rgimchakkana xavfidan xalos bo'ladi.

Zararkunandalar sonini kamaytirishda hasharotxo'r qushlarning ahamiyati ayniqsa yuqoridir. Qushlar hasharotlar kamayib ketgan dalalarni osonlik bilan topadi va zararkunandalarni yo'q qiladi.

Ko'sak qurti ko'paygan yozning 2-yarmida paxta dalalarida ko'plab qushlarning uchratish mumkin. Bog'larni qurtlardan xalos etishda chittaklar katta rol o'ynaydi. Bu kush bir kunda o'z vazniga barobar hasharotlarni qiradi. 1 jufti esa 40 tagacha mevali daraxtlarni zararkunandalardan bemalol himoya qila oladi. Bitta chug'urchuq oilasi jo'jalarini boqish uchun 8000 ga yaqin may qo'ng'izi va lichinkasiga qiron keltirishi mumkin. Faqatgina hasharotxo'rlargina emas, balki donxo'r qushlar ham (masalan, chumchuqlar) hasharotlar bilan ovqatlanadi. Shuning uchun ham foydali qushlarni muhofaza qilish, ularni ko'payishi uchun shart-sharoit yaratish muhim vazifalardan sanaladi. 
Biologik kurashning yana bir sohasi hasharotlarga qarshi mikrobiologik preparatlarni qo'llashdir. Bu usulda zararkanandalarda kasallik qo'zg'atuvchi bakteriyalar, viruslar va zamburug'lar foydalaniladi. Hozirda ko'sak qurtiga qarshi entobakteriya va dendrobatsellin deb ataladigan bakterial preparatlar qo'llanilmoqda. Bu preparatlar hasharotlarni 50 dan ortig'i samarali ta'sir etishini aniqlagan. Masalan, ular olma kuyasi, do'lana qurti, karam kuyasi, g'o'za va kuzgi tunlamlarga qarshi kurashda ishlatilmoqda. Bu preparatlar odam, hayvonlar va zararsiz hasharotlar uchun zararsizdir, ulardan hayvonlarni istalgan vegetatsiya fazasida, jumladan, gullash va hosilni olish davrida foydalanish mumkin.

Hasharotlar tanasida 1200 dan ortiq sodda hayvonlar va yumaloq chuvalchanglar parazitlik qilishi aniqlangan. Hozirda ularning ayrimlari biologik agent sifatida foydalanish ustida ishlar olib borilmoqda.

Yuqoridagilar asosida ta'kidlash mumkinki, biologik usul zararkunanda hasharotlarga qarshi qo'llanib kelinayotgan boshqa usullarni inkor etmaydi. Faqatgina o'simliklarni himoya etish jarayonida kimyoviy, fizikaviy, mexanik, agrotexnik va boshqa usullardagi bo'shliqlarni to'ldirishga xizmat qiladi.

Keyingi yillarda, ba'zi bir turlarni soni ortib bormoqda. Ular qatoriga tut va anor parvonalari, termitlar, ilonbosh baliq, "mayna", "kulrang kalamush" va boshqalarni kiritish mumkin. Ularning barchasi har bir turni chuqur o'rganishni, monitoring tizimini ishlab chiqishni taqazo etadi. 


\section{FOYDALANILGAN ADABIYOTLAR}

Bigon M., Xarper Dj., Taunsend K. Ekologiya. Osobi, populyatsiya i soobshestva. -M.: «Mir». Tom 1,2. 1989.

Vernadskiy V.I. Biosfera. Izbr. Trudi po biogeoximii. -M., 1967.

Vernadskiy V.I. Jivoe veshestvo. -M., 1978.

Viktorov G.A. Ekologiya parazitov entomofagov. -M., 1978.

Dajo R. Osnovi ekologii. -M.: «Progress», 1975.

Kashkarov D.N. Osnovi ekologii jivotnix. -M.-L., 1938: 2-s izd. isprav. i pererab. -L., 1945.

Naumov N.P. Ekologiya jivotnix. -M.: "Visshaya shkola”. 1963.

Odum Yu. Osnovi ekologii. -M., 1975.

Rasulov M. Markaziy Osiyo tabiatshunoslik fanlari tarixi. -Toshkent: «O'qituvchi», 1993.

Radkevich V.A. Ekologiya. -Minsk, 1983.

Tishler V. Selskoxozyaystvennaya ekologiya. -M., 1971.

To’xtaev A. Ekologiya. -Toshkent: «0'qituvchi», 1998.

Chernova N.M., Bilova A.M. Ekologiya. -M.: «Prosveshenie», 1981.

Yaxontov V.V. Ekologiya nasekomix. -M., 1969. 


\section{MUNDARIJA}

КИРИШ.

1-bo'lim. Hayvonlar ekologiyasi fan sifatida..........................................

2-bo'lim. Autoekologiya. Hayvonlarning yashash muhitlari va adaptatsiyalar

3-bo'lim. Populyatsiyalar ekologiyasi

4-bo'lim. Biotsenoz, biogeotsenoz va ekotizimlar. $92-104$

5-bo'lim. Biosferada hayvonlarning tarqalish chegaralari

Foydalanilgan adabiyotlar 